\title{
Tables for the Evaluation of the Faxén Approximation to the Solution of the Lamm Equation
}

\author{
Menachem Dishon* \\ Goddard Institute for Space Studies, National Aeronautics and Space Administration, \\ New York, N.Y., 10027 \\ and \\ George H. Weiss
}

National Cancer Institute, National Institutes of Health, Bethesda, Md., 20014

(November 5, 1965)

\begin{abstract}
A table is presented to facilitate the calculation of the Faxén approximation to the concentration and concentration gradient. The table is accurate to within one figure in the last place, and can be used both for no sedimentation dependence on concentration and for the dependence $s=s_{0}(1-k c)$.
\end{abstract}

Key Words: Faxén approximation, Lamm equation, ultracentrifuge, theory of noise, theory of ion exchange columns.

The Faxén approximation to the solution of the Lamm equation has proved to be of considerable utility in the analysis of data from velocity sedimentation experiments with the ultracentrifuge [1]. ${ }^{1} \quad$ It has recently been shown that the Faxén solution to the nonlinear Lamm equation which arises when the sedimentation coefficient $s$ is related to concentration $c$ by $s=s_{0}(1-k c)$ can be written in terms of the Faxén solution for $k=0$ [2]. Because of this wide range of applicability of the Faxén solution, it is appropriate to present a table to facilitate the calculation of concentration and concentration gradients in that approximation. The best known of earlier, equivalent tables, are those of Opler and Hiester [3]. Their tabulation gives very few values of the relevant function in the range of interest for ultracentrifuge work. A thorough analysis of mathematical properties of the function arising from the Faxén solution has been published by Goldstein [4]. Other applications of the tabulated function are to be found in the theory of noise [5], and in the theory of ion exchange columns [6].

Let us summarize the relevant facts on the Faxén solution. It is assumed that the concentration in the ultracentrifuge is $c(r, t)$, where $r$ is the radius and $t$ the time, with $c(r, 0)=c_{0}$, a constant. Let $r_{0}$ denote radial distance to the meniscus, $\omega$ the frequency of revolution, $D$ the diffusion coefficient. The Faxén solution is most conveniently written in terms of the new variables:

$\theta=c / c_{0}, \quad x=\left(r / r_{0}\right)^{2}, \quad \tau=2 \omega^{2} s_{0} t$,

$$
\alpha=k c_{0}, \quad \epsilon=2 D /\left(s_{0} \omega^{2} r_{0}^{2}\right), \quad z=2 \sqrt{x e^{-\tau}}, \quad \zeta=1-e^{-\tau} .
$$

When $k=0$, or equivalently, when $\alpha=0$, the Faxén expression for $\theta(z, \zeta)$ is

$$
\theta_{0}(z, \zeta)=\frac{1-\zeta}{2 \epsilon \zeta} \int_{2}^{\infty} \sigma e^{-\frac{\left(\sigma^{2}+z^{2}\right)}{4 \epsilon \zeta}} I_{0}\left(\frac{z \sigma}{2 \epsilon \zeta}\right) d \sigma
$$

*NAS-NRC Post-doctoral Research Associate with NASA. Permanent address: The Weizmann Institute of Science, Rehovath, Israel. 
When $\alpha>0$ we have

$$
\theta_{\alpha}(z, \zeta)=\left[\frac{1-\zeta}{1-(1+\alpha) \zeta}\right] \frac{\theta_{0}\left(z^{*}, \zeta^{*}\right) e^{-\frac{\alpha}{\epsilon}\left[1-\frac{z^{2}}{4(1-\alpha \zeta)}\right]}}{\left\{1+\frac{1-\alpha \zeta}{1-(1+\alpha) \zeta} \theta_{0}\left(z^{*}, \zeta^{*}\right) e^{-\frac{\alpha}{\epsilon}\left[1-\frac{z^{2}}{4(1-\alpha \zeta)}\right]}-\frac{1}{1-\zeta} \theta_{0}(z, \zeta)\right\}}
$$

where

$$
z^{*}=z(1-\alpha \zeta)^{-1}, \quad \zeta^{*}=\zeta(1-\alpha \zeta)^{-1} .
$$

Hence $\theta_{\alpha}(z, \zeta)$ can be represented in terms of $\theta_{0}(z, \zeta)$.

In the following pages we tabulate values of the functions

$$
\begin{gathered}
\psi(y, \eta)=\frac{1}{2 \eta} \int_{2}^{\infty} \sigma e^{-\frac{\left(\sigma^{2}+y^{2}\right)}{4 \eta}} I_{0}\left(\frac{\sigma y}{2 \eta}\right) d \sigma \\
\frac{\partial \psi(y, \eta)}{\partial y}=\frac{1}{4 \eta^{2}} \int_{2}^{\infty} \sigma e^{-\frac{\left(\sigma^{2}+y^{2}\right)}{4 \eta}}\left[\sigma I_{1}\left(\frac{\sigma y}{2 \eta}\right)-y I_{0}\left(\frac{\sigma y}{2 \eta}\right)\right] d \sigma
\end{gathered}
$$

accurate to at least four places with a possible error of 1 in the last place. The intervals of tabulation in $\eta$ are $\eta=10^{-6}\left(10^{-6}\right) 10^{-5}\left(10^{-5}\right) 10^{-4}\left(10^{-4}\right) 10^{-3}$. For each value of $\eta$ we choose from 28 to 80 steps in $y$ so that $\psi(y, \eta)$ lies in the range $(0.005,0.995)$.

The solutions for concentration and concentration gradients in terms of the tabulated functions are

$$
\begin{gathered}
\theta_{0}(z, \zeta)=(1-\zeta) \psi(z, \epsilon \zeta) \\
\frac{\partial \theta_{0}(z, \zeta)}{\partial\left(r / r_{0}\right)}=2(1-\zeta)^{3 / 2} \frac{\partial \psi(z, \epsilon \zeta)}{\partial z}
\end{gathered}
$$

for $\alpha=0$, and

$$
\begin{aligned}
\frac{\partial \theta_{\alpha}(z, \zeta)}{\partial\left(r / r_{0}\right)}=\frac{1}{(1-\alpha \zeta) F} \frac{\partial \theta_{0}(z, \zeta)}{\partial\left(r / r_{0}\right)}+\frac{1-\zeta-\theta_{0}(z, \zeta)}{(1-\alpha \zeta) F^{2}} \times\left\{\frac{1}{1-(1+\alpha) \zeta}\right. \\
\left.\quad\left[(1-\alpha \zeta) \frac{\partial \theta_{0}\left(z^{*}, \zeta^{*}\right)}{\partial\left(r / r_{0}\right)}+\frac{\alpha z}{\epsilon}(1-\zeta)^{1 / 2} \theta_{0}\left(z^{*}, \zeta *\right)\right] e^{-\frac{\alpha}{\epsilon}\left[1-\frac{z^{2}}{4(1-\alpha \zeta)}\right]}-\frac{1}{1-\zeta} \frac{\partial \theta_{0}(z, \zeta)}{\partial\left(r / r_{0}\right)}\right\}
\end{aligned}
$$

for $\alpha>0$, where

$$
F=1+\frac{1-\alpha \zeta}{1-(1+\alpha) \zeta} \theta_{0}\left(z^{*}, \zeta^{*}\right) e^{-\frac{\alpha}{\epsilon}\left[1-\frac{z^{2}}{4(1-\alpha \zeta)}\right]} \quad-\frac{1}{1-\zeta} \theta_{0}(z, \zeta)
$$

The integrals were evaluated using Simpson's rule. The interval of integration $h$ was varied from $2 \times 10^{-5}$ to $10^{-3}$ for different values of $y$ and $\eta$, so as always to meet the requirements of accuracy. The integration was stopped when the integrand was less than $10^{-25}$. As a check on our accuracy, the integration was performed twice; once over the interval $(2, \infty)$ and once over the 
interval $(0,2)$ with the appropriate integrals derived from the formulas

$$
\frac{1}{2 \eta} \int_{2}^{\infty} \sigma e^{-\frac{\left(\sigma^{2}+y^{2}\right)}{4 \eta}} I_{0}\left(\frac{\sigma y}{2 \eta}\right) d \sigma=1-\frac{1}{2 \eta} \int_{0}^{2} \sigma e^{-\frac{\left(\sigma^{2}+y^{2}\right)}{4 \eta}} I_{0}\left(\frac{\sigma y}{2 \eta}\right) d \sigma
$$

and

$$
\int_{2}^{\infty} \sigma e^{-\frac{\left(\sigma^{2}+y^{2}\right)}{4 \eta}}\left[\sigma I_{1}\left(\frac{\sigma y}{2 \eta}\right)-y I_{0}\left(\frac{\sigma y}{2 \eta}\right)\right] d \sigma=-\int_{0}^{2} \sigma e^{-\frac{\left(\sigma^{2}+y^{2}\right)}{4 \eta}}\left[\sigma I_{1}\left(\frac{\sigma y}{2 \eta}\right)-y I_{0}\left(\frac{\sigma y}{2 \eta}\right)\right] d \sigma
$$

Since the argument of the Bessel functions was always greater than 1200 , the asymptotic expansions

$$
\begin{aligned}
& I_{0}(x) \sim \frac{e^{x}}{\sqrt{2 \pi x}}\left[1+\frac{1}{1 !(8 x)}+\frac{3^{2}}{2 !(8 x)^{2}}+\frac{3^{2} 5^{2}}{3 !(8 x)^{3}}+\ldots\right] \\
& I_{1}(x) \sim \frac{e^{x}}{\sqrt{2 \pi x}}\left[1-\frac{3}{1 ! 8 x}-\frac{3.5}{2 !(8 x)^{2}}-\frac{325.7}{3 !(8 x)^{3}}-\ldots\right]
\end{aligned}
$$

were used to evaluate these Bessel functions.

All the calculations are accurate to within one figure in the fifth place. Eight place tables

\begin{tabular}{|c|c|c|c|c|c|c|c|c|}
\hline$Y$ & $P S I(Y, E T A)$ & DPSI I Y,ETA & I/DY & $Y$ & PSI IY, EI & (A) & DPS I $(Y, E T$ & A) / DY \\
\hline $\begin{array}{l}1.9960 \\
1.9965 \\
1.9970 \\
1.9975 \\
1.9980\end{array}$ & $\begin{array}{l}0.23414 D-02 \\
0.66707 D-02 \\
0.16962 D-01 \\
0.38579 D-01 \\
0.787010-01\end{array}$ & $\begin{array}{l}0.517180 \\
0.132050 \\
0.297550 \\
0.591670 \\
0.103830\end{array}$ & $\begin{array}{l}01 \\
02 \\
02 \\
02 \\
03\end{array}$ & $\begin{array}{l}2.0002 \\
2.0004 \\
2.0006 \\
2.0008 \\
2.0010\end{array}$ & $\begin{array}{l}0.556370 \\
0.611490 \\
0.664440 \\
0.714320 \\
0.760360\end{array}$ & $\begin{array}{l}00 \\
00 \\
00 \\
00 \\
00\end{array}$ & $\begin{array}{l}0.279270 \\
0.271010 \\
0.257780 \\
0.240340 \\
0.219640\end{array}$ & $\begin{array}{l}03 \\
03 \\
03 \\
03 \\
03\end{array}$ \\
\hline $\begin{array}{l}1.9982 \\
1.9984 \\
1.9986 \\
1.9988 \\
1.9990\end{array}$ & $\begin{array}{l}0.101610-00 \\
0.129020-00 \\
0.161190-00 \\
0.198170-00 \\
0.239860-00\end{array}$ & $\begin{array}{l}0.125550 \\
0.148810 \\
0.172880 \\
0.196870 \\
0.219750\end{array}$ & $\begin{array}{l}03 \\
03 \\
03 \\
03 \\
03\end{array}$ & $\begin{array}{l}2.0012 \\
2.0014 \\
2.0016 \\
2.0018 \\
2.0020\end{array}$ & $\begin{array}{l}0.802030 \\
0.838990 \\
0.871120 \\
0.898520 \\
0.921400\end{array}$ & $\begin{array}{l}00 \\
00 \\
00 \\
00 \\
00\end{array}$ & $\begin{array}{l}0.196750 \\
0.172760 \\
0.148690 \\
0.125440 \\
0.103730\end{array}$ & $\begin{array}{l}03 \\
03 \\
03 \\
03 \\
03\end{array}$ \\
\hline $\begin{array}{l}1.9992 \\
1.9994 \\
1.9996 \\
1.9998 \\
2.0000\end{array}$ & $\begin{array}{l}0.28592 D-00 \\
0.33581 D-00 \\
0.38878 D-00 \\
0.443910-00 \\
0.50014 D \quad 00\end{array}$ & $\begin{array}{l}0.240430 \\
0.257850 \\
0.271060 \\
0.279300 \\
0.282090\end{array}$ & $\begin{array}{l}03 \\
03 \\
03 \\
03 \\
03\end{array}$ & $\begin{array}{l}2.0022 \\
2.0025 \\
2.0030 \\
2.0035 \\
2.0040\end{array}$ & $\begin{array}{l}0.940140 \\
0.961480 \\
0.983070 \\
0.993340 \\
0.997660\end{array}$ & $\begin{array}{l}00 \\
00 \\
00 \\
00 \\
00\end{array}$ & $\begin{array}{l}0.84076 \mathrm{C} \\
0.59093 \mathrm{C} \\
0.29710 \mathrm{C} \\
0.13182 \mathrm{C} \\
0.51616 \mathrm{C}\end{array}$ & $\begin{array}{l}02 \\
02 \\
02 \\
02 \\
01\end{array}$ \\
\hline
\end{tabular}
are available from the authors, as well as a tabulation for the range $0.001<\eta \leqslant 0.01$.

TABLE FOR THE EVALUATION OF THE FAXEN APPROXIMATION TO THE SOLUTION OF THE LAMM EQUATICN

$$
E T A=0.000001
$$


$E T A=0.000002$

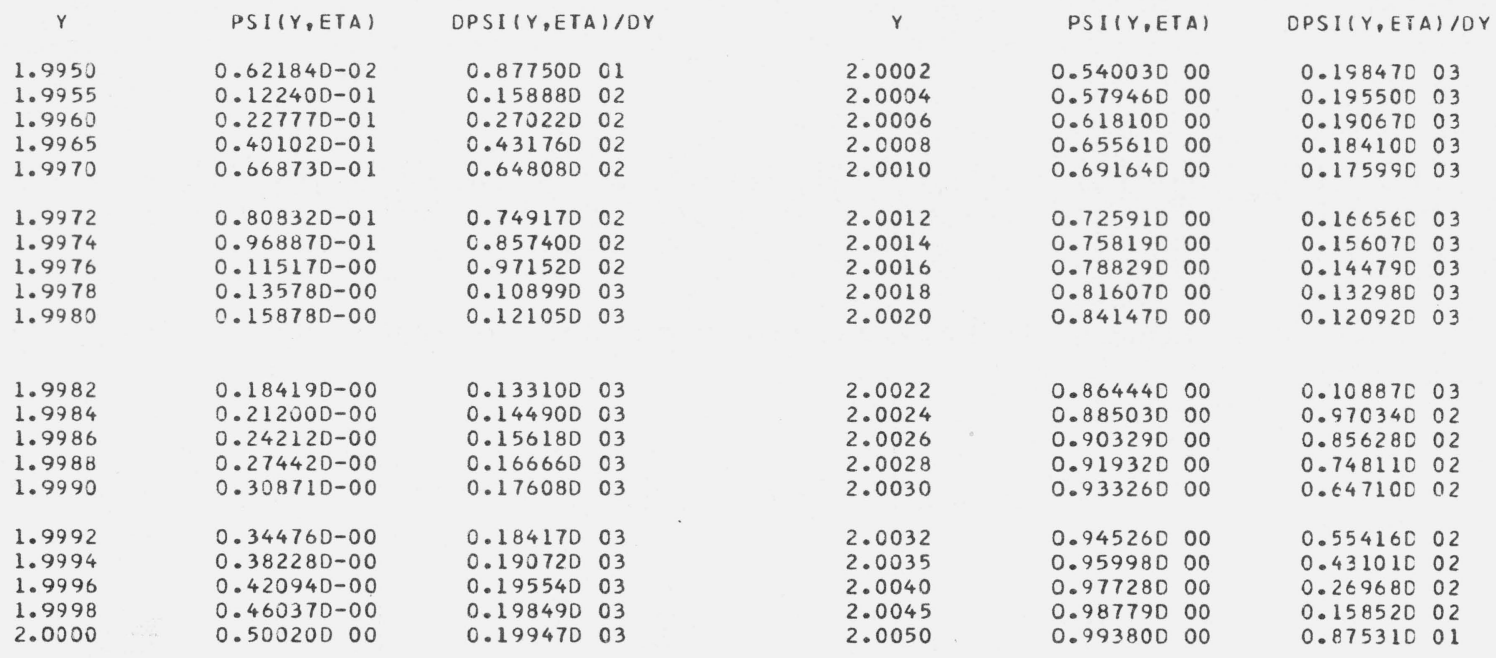

TABLE FOR THE EVALUATION OF THE FAXEN APPROXIMATION TO THE SOLUTION OF THE LAMM EQUATICN

$E T A=0.000003$

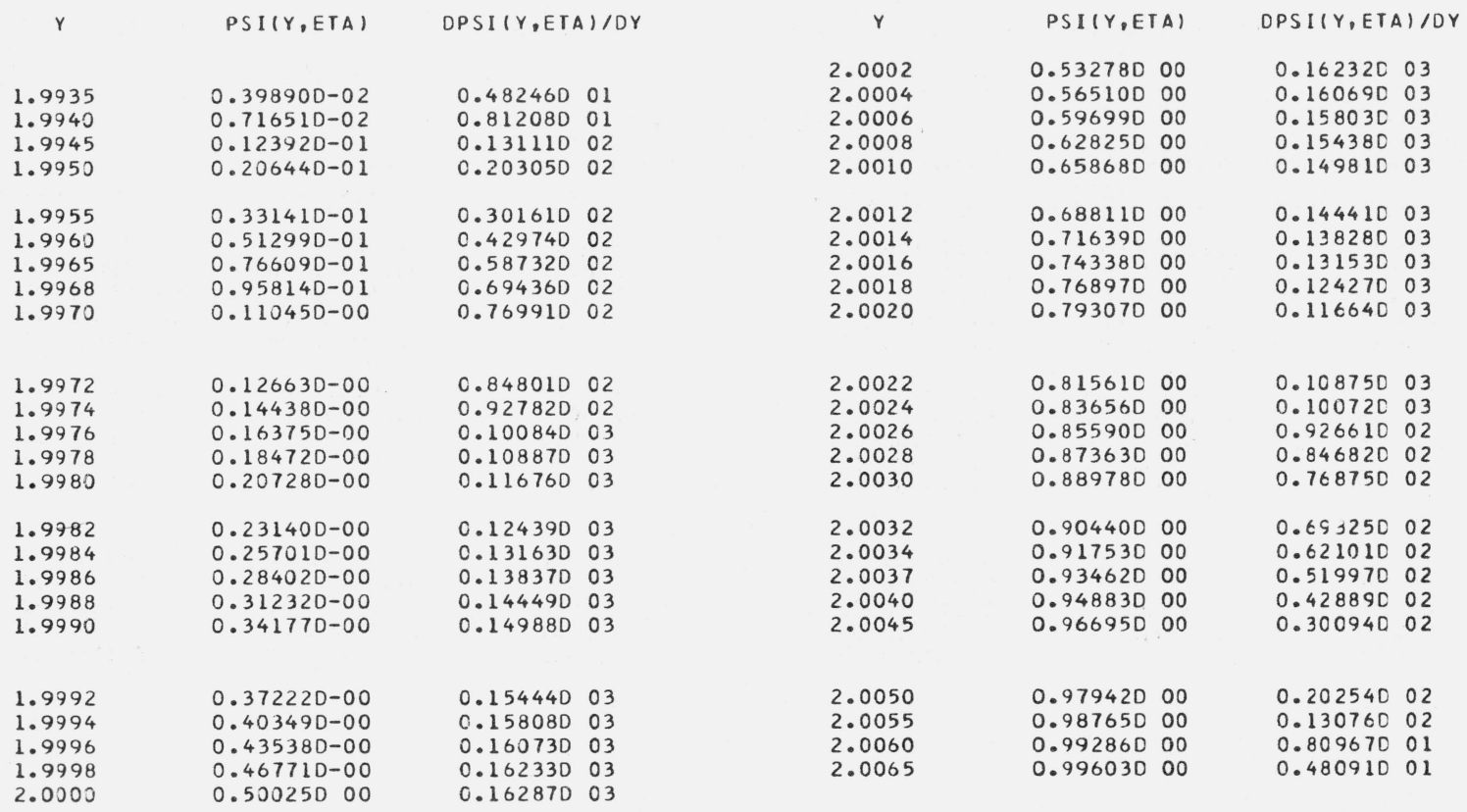


$E T A=0.000004$

1.9996

1. 9998

2.0000

2.0002
2.0004
2.0006
2.0008
2.0010
2.0012
2.0014
2.0016
2.0018
2.0020

$0.572570 \quad 02$ 0.62803002 0.68542002

0.74433002

0.80427002

0.86470002

0.92504002

$0.984650 \quad 02$

0.10429003

0.10990003

$0.115240 \quad 03$

0.12024003

0.12483003

0.12895003

$0.132530 \quad 03$

$0.13554 \mathrm{D} 03$

0.13793003

0.13966003

0.14070003

0.14105003
0.52847000 0.55651000 $0.584270 \quad 00$ 0.61162000 0.63843000

0.66457000 $0.68994 \mathrm{D} 00$ 0.71444000 0.73797000 0.76047000

2.0022

2.0024

2.0026

2.0028

2.0030

2.0032

2.0034

2.0036

2. 0038

2. 0040

2.0042

2. 0045

2.0050

2.0055

2.0060

2.0065

2.0070

$0.781870 \quad 00$ 0.80213000 0.82120000 0.83907000 $0.855740 \quad 00$
$0.14069 D 03$ 0.13963003 0.13789003 0.13549003 $0.132470 \quad 03$

$0.12887 C_{03}$ $0.124740 \quad 03$ 0.12014003

0.11514003 0.10979003

0.10417003 $0.983460 \quad 02$ 0.92383002 $0.863490 \quad 02$ 0.80306002

$0.743140 \quad 02$ 0.68425002 0.62689002 $0.57148 D \quad 02$ 0.51836002 $0.397400 \quad 02$ $0.29528 \mathrm{D} 02$ 0.21266002 $0.14844 \mathrm{DO} 02$

0.10043002 $0.65854 \mathrm{C} 01$
$0.467840 \quad 02$ 
$E T A=0.000005$

\begin{tabular}{|c|c|c|c|c|c|c|c|c|}
\hline$Y$ & $P S I(Y, E T A)$ & DPSI $(Y, E T A$ & ) /DY & $Y$ & PSI $(Y, E T$ & A) & DPSI $I Y, E T A$ & A) $/ D$ \\
\hline $\begin{array}{l}1.9920 \\
1.9925 \\
1.9930 \\
1.9935 \\
1.9940\end{array}$ & $\begin{array}{l}0.571890-02 \\
0.887200-02 \\
0.134560-01 \\
0.199550-01 \\
0.28942 D-01\end{array}$ & $\begin{array}{l}0.515270 \\
0.759050 \\
0.109060 \\
0.152820 \\
0.208850\end{array}$ & $\begin{array}{l}01 \\
01 \\
02 \\
02 \\
02\end{array}$ & $\begin{array}{l}2.0002 \\
2.0004 \\
2.0006 \\
2.0008 \\
2.0010\end{array}$ & $\begin{array}{l}0.52553 D \\
0.55064 D \\
0.57555 D \\
0.60016 D \\
0.62438 D\end{array}$ & $\begin{array}{l}00 \\
00 \\
00 \\
00 \\
00\end{array}$ & $\begin{array}{l}0.125900 \\
0.125140 \\
0.123890 \\
0.122160 \\
0.119970\end{array}$ & $\begin{array}{l}03 \\
03 \\
03 \\
03 \\
03\end{array}$ \\
\hline $\begin{array}{l}1.9945 \\
1.9950 \\
1.9955 \\
1.9958 \\
1.9960\end{array}$ & $\begin{array}{l}0.410650-01 \\
0.570140-01 \\
0.774790-01 \\
0.921930-01 \\
0.103090-00\end{array}$ & $\begin{array}{l}0.278380 \\
0.361900 \\
0.458860 \\
0.522780 \\
0.567420\end{array}$ & $\begin{array}{l}02 \\
02 \\
02 \\
02 \\
02\end{array}$ & $\begin{array}{l}2.0012 \\
2.0014 \\
2.0016 \\
2.0018 \\
2.0020\end{array}$ & $\begin{array}{l}0.64812 D \\
0.67130 D \\
0.69384 D \\
0.71566 D \\
0.736710\end{array}$ & $\begin{array}{l}00 \\
00 \\
00 \\
00 \\
00\end{array}$ & $\begin{array}{l}0.117360 \\
0.114340 \\
0.110960 \\
0.107240 \\
0.103240\end{array}$ & $\begin{array}{l}03 \\
03 \\
03 \\
03 \\
03\end{array}$ \\
\hline $\begin{array}{l}1.9962 \\
1.9964 \\
1.9966 \\
1.9968 \\
1.9970\end{array}$ & $\begin{array}{l}0.11490 D-00 \\
0.12764 D-00 \\
0.14132 D-00 \\
0.155970-00 \\
0.171590-00\end{array}$ & $\begin{array}{l}0.613420 \\
0.660510 \\
0.708360 \\
0.756650 \\
0.805010\end{array}$ & $\begin{array}{l}02 \\
02 \\
02 \\
02 \\
02\end{array}$ & $\begin{array}{l}2.0022 \\
2.0024 \\
2.0026 \\
2.0028 \\
2.0030\end{array}$ & $\begin{array}{l}0.75694 D \\
0.77629 D \\
0.79474 D \\
0.812250 \\
0.828810\end{array}$ & $\begin{array}{l}00 \\
00 \\
00 \\
00 \\
00\end{array}$ & $\begin{array}{l}0.989860 \\
0.945310 \\
0.899160 \\
0.851850 \\
0.803810\end{array}$ & $\begin{array}{l}02 \\
02 \\
02 \\
02 \\
02\end{array}$ \\
\hline $\begin{array}{l}1.9972 \\
1.9974 \\
1.9976 \\
1.9978 \\
1.9980\end{array}$ & $\begin{array}{l}0.188170-00 \\
0.205710-00 \\
0.224180-00 \\
0.243550-00 \\
0.263800-00\end{array}$ & $\begin{array}{l}0.85304 D \\
0.900330 \\
0.946440 \\
0.990940 \\
0.10334 D\end{array}$ & $\begin{array}{l}02 \\
02 \\
02 \\
02 \\
03\end{array}$ & $\begin{array}{l}2.0032 \\
2.0034 \\
2.0036 \\
2.0038 \\
2.0040\end{array}$ & $\begin{array}{l}0.844400 \\
0.859030 \\
0.872690 \\
0.885410 \\
0.897190\end{array}$ & $\begin{array}{l}00 \\
00 \\
00 \\
00 \\
00\end{array}$ & $\begin{array}{l}0.755450 \\
0.707160 \\
0.659320 \\
0.612260 \\
0.566290\end{array}$ & $\begin{array}{l}02 \\
02 \\
02 \\
02 \\
02\end{array}$ \\
\hline $\begin{array}{l}1.9982 \\
1.9984 \\
1.9986 \\
1.9988 \\
1.9790\end{array}$ & $\begin{array}{l}0.284870-00 \\
0.306720-00 \\
0.329270-00 \\
0.352460-00 \\
0.376210-00\end{array}$ & $\begin{array}{l}0.107340 \\
0.111040 \\
0.1114420 \\
0.1117430 \\
0.1120030\end{array}$ & $\begin{array}{l}03 \\
03 \\
03 \\
03 \\
03\end{array}$ & $\begin{array}{l}2.0042 \\
2.0044 \\
2.0047 \\
2.0050 \\
2.0055\end{array}$ & $\begin{array}{l}0.908070 \\
0.918070 \\
0.931500 \\
0.943170 \\
0.959070\end{array}$ & $\begin{array}{l}00 \\
00 \\
00 \\
00 \\
00\end{array}$ & $\begin{array}{l}0.521680 \\
0.478670 \\
0.417560 \\
0.360990 \\
0.277620\end{array}$ & $\begin{array}{l}02 \\
02 \\
02 \\
02 \\
02\end{array}$ \\
\hline $\begin{array}{l}1.9992 \\
1.9994 \\
1.9996 \\
1.9998 \\
2.0500\end{array}$ & $\begin{array}{l}0.400450-00 \\
0.425070-00 \\
0.449980-00 \\
0.475100-00 \\
0.50031000\end{array}$ & $\begin{array}{l}0.122210 \\
0.123920 \\
0.125160 \\
0.125910 \\
0.126160\end{array}$ & $\begin{array}{l}03 \\
03 \\
03 \\
03 \\
03\end{array}$ & $\begin{array}{l}2.0060 \\
2.0065 \\
2.0070 \\
2.0075 \\
2.0080\end{array}$ & $\begin{array}{l}0.971160 \\
0.980120 \\
0.986600 \\
0.991170 \\
0.994310\end{array}$ & $\begin{array}{l}00 \\
00 \\
00 \\
00 \\
00\end{array}$ & $\begin{array}{l}0.208220 \\
0.152320 \\
0.108680 \\
0.756210 \\
0.513220\end{array}$ & $\begin{array}{l}02 \\
02 \\
02 \\
01 \\
01\end{array}$ \\
\hline
\end{tabular}


$E T A=0.000006$

Y

PSI (Y,ETA) DPSI $(Y, E T A) / D Y$

0.39496001

1.9910

1.9915

1. 9920

1.9925

1.9930

1.9935

1.9940

1.9945

1. 9750

1.9953

1. 9956

1. 9958

1.9760

1. 9962

1. 9964

1.9766

1. 9968

1.9970

1. 9972

1. 9974

1.9976

1. 9778

1. 9980

1. 9982

1. 9784

1. 9986

1. 9988

1. 9990

1. 9992

1. 9994

1.9796

1. 9998

2.0500
$0.469920-02$

$0.708600-02$

$0.104850-01$

$0.152250-01$

$0.216990-01$

$0.303600-01$

$0.417090-01$

$0.562740-01$

$0.745790-01$

$0.875640-01$

$0.102170-00$

$0.112840-00$

$0.124280-00$

$0.136520-00$

$0.149550-00$

$0.163390-00$.

$0.178030-00$

$0.193480-00$

$0.209710-00$

$0.226720-00$

$0.244480-00$

$0.262970-00$

$0.282140-00$

$0.301970-00$

0. 322390-00

$0.343370-00$

$0.364840-00$

$0.386750-00$

$0.409020-00$

$0.431590-00$

$0.454380-00$

$0.477320-00$

0.50035000
0.56864001

0.80181001

0.11373002

$0.149760 \quad 02$

0.19837002

0.25735002

0.32698002

0.40689002

0.51460002

$0.552800 \quad 02$

C.591870 02

0.63158002

$0.67172 \mathrm{D} \quad 02$

0.71204002

0.75226002

0.79211002

$0.831290 \quad 02$

$0.86951 D 02$

$0.906460 \mathrm{C2}$

0.94184002

0.97533002

0.10067003

0.10355003

0.10617003

0.10849003

C.11049D 03

0.11216003

0.11347003

0.11441003

0.11498003

0.11516003
0.45930002
PSI (Y, ETA)

0.52336000

0.54631000

0.56910000

0.59166000

0.61392000

0.63581000

0.65727000

0.67823000

$0.69864 D \quad 00$

0.71844000

$0.737600 \quad 00$

0.75606000

0.77380000

0.79079000

0.80700000

0.82242000

$0.83704 \mathrm{D} 00$

$0.850850 \quad 00$

0.86386000

0.87607000

2. 0038

2.0040

2.0042

2.0044

2.0046

2.0048

2.0050

2.0055

2.0060

2.0065

2.0070

2.0075

2.0080

2.0085

2.0090
0.88749000

0.89814000

0.90804000

0.91720000

0.92566000

$0.943920 \quad 00$

0.95844000

$0.96976 \mathrm{D} 00$

$0.978390 \quad 00$

0.98484000

0.98956000

0.99295000

0.99532000
DPSI (Y, ETA)/DY

$0.114970 \quad 03$

$0.114390 \quad 03$

0.11343003

0.11211003

$0.110440 \quad 03$

0.10843003

0.10610003

0.10347003

0.10058003

0.97436002

0.94080002

0.90537002

0.86838002

0.83013002

0.79092002

$0.751060 \quad 02$

$0.710830 \quad 02$

$0.670520 \quad 02$

0.63039002

0.59068002

$0.551640 \quad 02$

$0.51347 \mathrm{C} 02$

$0.476340 \quad 02$

$0.440430 \quad 02$

0.40587002

$0.32608 \mathrm{C} 02$

$0.256580 \quad 02$

0.19773002

0.14924002

0.11031002

$0.798600 \quad 01$

0.56622001

0.39318001 
$E T A=0.000007$

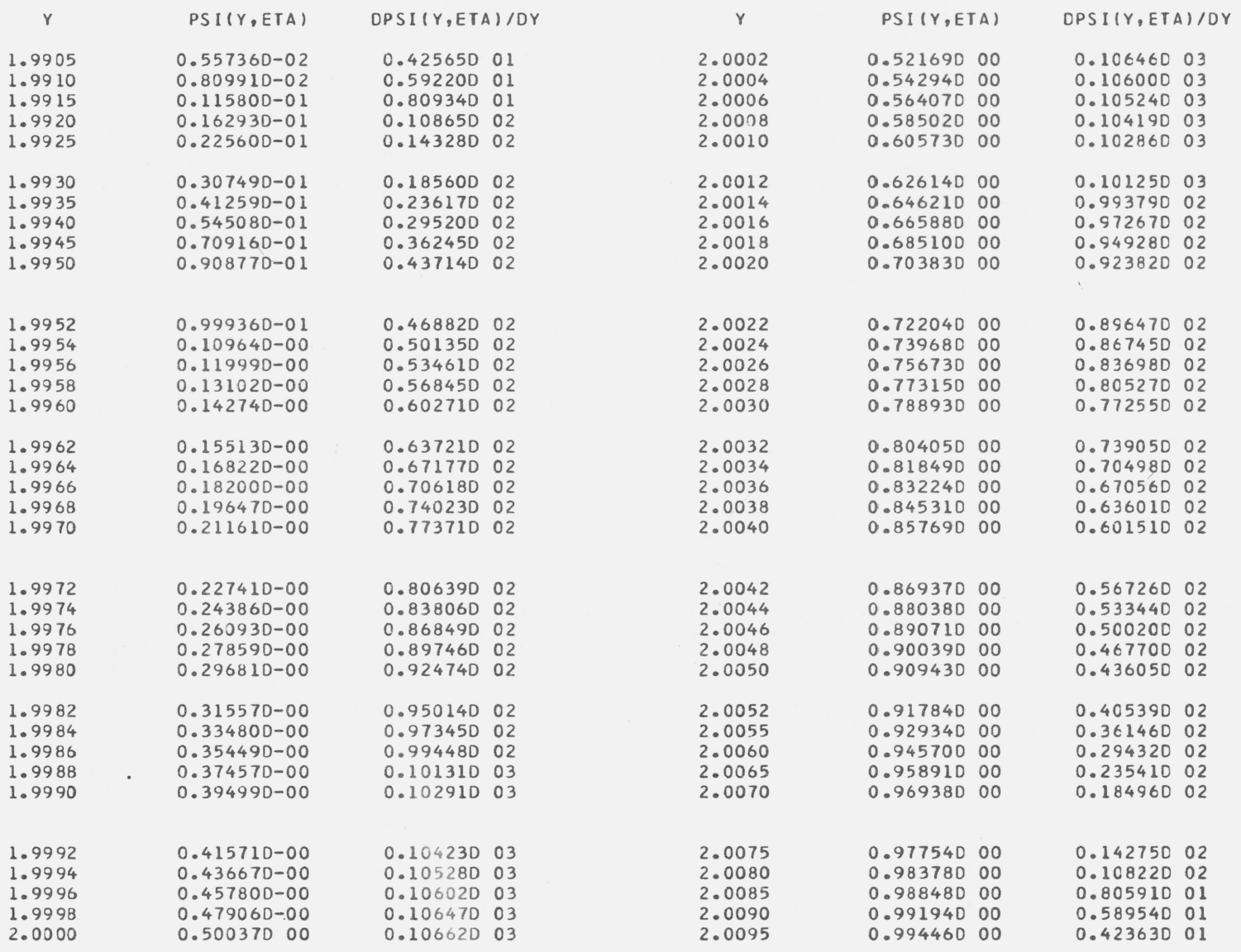


$E T A=0.000008$

\begin{tabular}{|c|c|c|c|c|c|c|c|c|}
\hline Y & $\operatorname{PSI}(Y, E T A)$ & DPSI $Y, E T$ & A) $/ D Y$ & $Y$ & PSI $(Y, E$ & TA) & DPSI $(Y, E T$ & A) /DY \\
\hline $\begin{array}{l}1.9895 \\
1.9700\end{array}$ & $\begin{array}{l}0.43452 D-02 \\
0.62272 D-02\end{array}$ & $\begin{array}{l}0.318940 \\
0.439300\end{array}$ & $\begin{array}{l}01 \\
01\end{array}$ & $\begin{array}{l}2.0002 \\
2.0004 \\
2.0006 \\
2.0008 \\
2.0010\end{array}$ & $\begin{array}{l}0.520340 \\
0.540220 \\
0.560010 \\
0.579650 \\
0.599090\end{array}$ & $\begin{array}{l}00 \\
00 \\
00 \\
00 \\
00\end{array}$ & $\begin{array}{l}0.996060 \\
0.992280 \\
0.986050 \\
0.977410 \\
0.966430\end{array}$ & $\begin{array}{l}02 \\
02 \\
02 \\
02 \\
02\end{array}$ \\
\hline $\begin{array}{l}1.9905 \\
1.9910 \\
1.9915 \\
1.9923 \\
1.9925\end{array}$ & $\begin{array}{l}0.879820-02 \\
0.122560-01 \\
0.168350-01 \\
0.228040-01 \\
0.304650-01\end{array}$ & $\begin{array}{l}0.595710 \\
0.795280 \\
0.104520 \\
0.135250 \\
0.172290\end{array}$ & $\begin{array}{l}01 \\
01 \\
02 \\
02 \\
02\end{array}$ & $\begin{array}{l}2.0012 \\
2.0014 \\
2.0016 \\
2.0018 \\
2.0020\end{array}$ & $\begin{array}{l}0.618290 \\
0.637210 \\
0.655790 \\
0.674000 \\
0.691810\end{array}$ & $\begin{array}{l}00 \\
00 \\
00 \\
00 \\
00\end{array}$ & $\begin{array}{l}0.953180 \\
0.937770 \\
0.920310 \\
0.900910 \\
0.879720\end{array}$ & $\begin{array}{l}02 \\
02 \\
02 \\
02 \\
02\end{array}$ \\
\hline $\begin{array}{l}1.9930 \\
1.9935 \\
1.9940 \\
1.9945 \\
1.9950\end{array}$ & $\begin{array}{l}0.401450-01 \\
0.521880-01 \\
0.669370-01 \\
0.847210-01 \\
0.105830-00\end{array}$ & $\begin{array}{l}0.216070 \\
0.266780 \\
0.324280 \\
0.388060 \\
0.457190\end{array}$ & $\begin{array}{l}02 \\
02 \\
02 \\
02 \\
02\end{array}$ & $\begin{array}{l}2.0022 \\
2.0024 \\
2.0026 \\
2.0028 \\
2.0030\end{array}$ & $\begin{array}{l}0.709180 \\
0.726080 \\
0.742480 \\
0.758350 \\
0.773670\end{array}$ & $\begin{array}{l}00 \\
00 \\
00 \\
00 \\
00\end{array}$ & $\begin{array}{l}0.856890 \\
0.832560 \\
0.806910 \\
0.780090 \\
0.752280\end{array}$ & $\begin{array}{l}02 \\
02 \\
02 \\
02 \\
02\end{array}$ \\
\hline $\begin{array}{l}1.9752 \\
1.9954 \\
1.9956 \\
1.9958 \\
1.9960\end{array}$ & $\begin{array}{l}0.115260-00 \\
0.125280-00 \\
0.135880-00 \\
0.147090-00 \\
0.158900-00\end{array}$ & $\begin{array}{l}0.486050 \\
0.515430 \\
0.545230 \\
0.575310 \\
0.605530\end{array}$ & $\begin{array}{l}02 \\
02 \\
02 \\
02 \\
02\end{array}$ & $\begin{array}{l}2.0032 \\
2.0034 \\
2.0036 \\
2.0038 \\
2.0040\end{array}$ & $\begin{array}{l}0.788430 \\
0.802610 \\
0.816210 \\
0.829200 \\
0.841590\end{array}$ & $\begin{array}{l}00 \\
00 \\
00 \\
00 \\
00\end{array}$ & $\begin{array}{l}0.723650 \\
0.694370 \\
0.664610 \\
0.634540 \\
0.604320\end{array}$ & $\begin{array}{l}02 \\
02 \\
02 \\
02 \\
02\end{array}$ \\
\hline $\begin{array}{l}1.9962 \\
1.9764 \\
1.9966 \\
1.9968 \\
1.9970\end{array}$ & $\begin{array}{l}0.171310-00 \\
0.184330-00 \\
0.197940-00 \\
0.212150-00 \\
0.226930-00\end{array}$ & $\begin{array}{l}0.635750 \\
0.665810 \\
0.695550 \\
0.724810 \\
0.753410\end{array}$ & $\begin{array}{l}02 \\
02 \\
02 \\
02 \\
02\end{array}$ & $\begin{array}{l}2.0042 \\
2.0044 \\
2.0046 \\
2.0048 \\
2.0050\end{array}$ & $\begin{array}{l}0.853370 \\
0.864550 \\
0.875130 \\
0.885120 \\
0.894530\end{array}$ & $\begin{array}{l}00 \\
00 \\
00 \\
00 \\
00\end{array}$ & $\begin{array}{l}0.574100 \\
0.544030 \\
0.514250 \\
0.484880 \\
0.456050\end{array}$ & $\begin{array}{l}02 \\
02 \\
02 \\
02 \\
02\end{array}$ \\
\hline $\begin{array}{l}1.9972 \\
1.9974 \\
1.9976 \\
1.9978 \\
1.9980\end{array}$ & $\begin{array}{l}0.242280-00 \\
0.258170-00 \\
0.27459 D-00 \\
0.29150 D-00 \\
0.30889 D-00\end{array}$ & $\begin{array}{l}0.781180 \\
0.807950 \\
0.833560 \\
0.857830 \\
0.880600\end{array}$ & $\begin{array}{l}02 \\
02 \\
02 \\
02 \\
02\end{array}$ & $\begin{array}{l}2.0052 \\
2.0055 \\
2.0060 \\
2.0065 \\
2.0070\end{array}$ & $\begin{array}{l}0.903370 \\
0.915590 \\
0.933320 \\
0.948030 \\
0.960030\end{array}$ & $\begin{array}{l}00 \\
00 \\
00 \\
00 \\
00\end{array}$ & $\begin{array}{l}0.427860 \\
0.38700 D \\
0.323310 \\
0.265910 \\
0.215320\end{array}$ & $\begin{array}{l}02 \\
02 \\
02 \\
02 \\
02\end{array}$ \\
\hline $\begin{array}{l}1.9982 \\
1.9984 \\
1.9986 \\
1.9988 \\
1.9990\end{array}$ & $\begin{array}{l}0.32672 D-00 \\
0.34495 D-00 \\
0.36354 D-00 \\
0.38247 D-00 \\
0.40168 D-00\end{array}$ & $\begin{array}{l}0.901720 \\
0.921040 \\
0.938430 \\
0.953750 \\
0.966910\end{array}$ & $\begin{array}{l}02 \\
02 \\
02 \\
02 \\
02\end{array}$ & $\begin{array}{l}2.0075 \\
2.0080 \\
2.0085 \\
2.0090 \\
2.0095\end{array}$ & $\begin{array}{l}0.969670 \\
0.977300 \\
0.983250 \\
0.987810 \\
0.991250\end{array}$ & $\begin{array}{l}00 \\
00 \\
00 \\
00 \\
00\end{array}$ & $\begin{array}{l}0.171640 \\
0.134710 \\
0.104080 \\
0.791700 \\
0.592890\end{array}$ & $\begin{array}{l}02 \\
02 \\
02 \\
01 \\
01\end{array}$ \\
\hline $\begin{array}{l}1.9992 \\
1.9994 \\
1.9996 \\
1.9998 \\
2.0000\end{array}$ & $\begin{array}{l}0.421130-00 \\
0.440780-00 \\
0.46057 D-00 \\
0.480460-00 \\
0.50040000\end{array}$ & $\begin{array}{l}0.97780 D \\
0.98634 \mathrm{D} \\
0.992480 \\
0.99616 \mathrm{D} \\
0.99735 \mathrm{D}\end{array}$ & $\begin{array}{l}02 \\
02 \\
02 \\
02 \\
02\end{array}$ & $\begin{array}{l}2.0100 \\
2.0105\end{array}$ & $\begin{array}{l}0.993810 \\
0.995680\end{array}$ & $\begin{array}{l}00 \\
00\end{array}$ & $\begin{array}{l}0.437110 \\
0.317270\end{array}$ & $\begin{array}{l}01 \\
01\end{array}$ \\
\hline
\end{tabular}


$E T A=0.000009$

\begin{tabular}{|c|c|c|c|c|c|c|c|c|}
\hline Y & $P S I(Y, E T A)$ & DPSI I Y,ETA & A) $/ D Y$ & Y & PSI I Y,ET & & DPS I $(Y, E T$ & A) $/ 0$ \\
\hline $\begin{array}{l}1.9890 \\
1.9895 \\
1.9900 \\
1.9905\end{array}$ & $\begin{array}{l}0.477570-02 \\
0.66840 D-02 \\
0.923740-02 \\
0.126070-01\end{array}$ & $\begin{array}{l}0.327160 \\
0.440950 \\
0.586120 \\
0.768340\end{array}$ & $\begin{array}{l}01 \\
01 \\
01 \\
01\end{array}$ & $\begin{array}{l}2.0002 \\
2.0004 \\
2.0006 \\
2.0008 \\
2.0010\end{array}$ & $\begin{array}{l}0.519220 \\
0.537980 \\
0.556650 \\
0.575200 \\
0.593580\end{array}$ & $\begin{array}{l}00 \\
00 \\
00 \\
00 \\
00\end{array}$ & $\begin{array}{l}0.939220 \\
0.936050 \\
0.930820 \\
0.923560 \\
0.914330\end{array}$ & $\begin{array}{l}02 \\
02 \\
02 \\
02 \\
02\end{array}$ \\
\hline $\begin{array}{l}1.9910 \\
1.9915 \\
1.9920 \\
1.9925 \\
1.9930\end{array}$ & $\begin{array}{l}0.16992 \mathrm{D}-01 \\
0.22620 \mathrm{D}-01 \\
0.29745 \mathrm{D}-01 \\
0.386390-01 \\
0.49589 \mathrm{D}-01\end{array}$ & $\begin{array}{l}0.993320 \\
0.126650 \\
0.159240 \\
0.197470 \\
0.241500\end{array}$ & $\begin{array}{l}01 \\
02 \\
02 \\
02 \\
02\end{array}$ & $\begin{array}{l}2.0012 \\
2.0014 \\
2.0016 \\
2.0018 \\
2.0020\end{array}$ & $\begin{array}{l}0.611760 \\
0.629690 \\
0.647350 \\
0.664700 \\
0.681700\end{array}$ & $\begin{array}{l}00 \\
00 \\
00 \\
00 \\
00\end{array}$ & $\begin{array}{l}0.903170 \\
0.890180 \\
0.875420 \\
0.859000 \\
0.841010\end{array}$ & $\begin{array}{l}02 \\
02 \\
02 \\
02 \\
02\end{array}$ \\
\hline & - & & & & & & & \\
\hline $\begin{array}{l}1.9935 \\
1.994 J \\
1.9945 \\
1.9948 \\
1.9950\end{array}$ & $\begin{array}{l}0.62884 D-01 \\
0.788050-01 \\
0.976090-01 \\
0.110360-00 \\
0.1119510-00\end{array}$ & $\begin{array}{l}0.291260 \\
0.346440 \\
0.406390 \\
0.444260 \\
0.470140\end{array}$ & $\begin{array}{l}02 \\
02 \\
02 \\
02 \\
02\end{array}$ & $\begin{array}{l}2.0022 \\
2.0024 \\
2.0026 \\
2.0028 \\
2.0030\end{array}$ & $\begin{array}{l}0.698330 \\
0.714560 \\
0.730350 \\
0.745700 \\
0.760580\end{array}$ & $\begin{array}{l}00 \\
00 \\
00 \\
00 \\
00\end{array}$ & $\begin{array}{l}0.821570 \\
0.800800 \\
0.778830 \\
0.755770 \\
0.731770\end{array}$ & $\begin{array}{l}02 \\
02 \\
02 \\
02 \\
02\end{array}$ \\
\hline $\begin{array}{l}1.9952 \\
1.9954 \\
1.9956 \\
1.9958 \\
1.9960\end{array}$ & $\begin{array}{l}0.129170-00 \\
0.139370-00 \\
0.150090-00 \\
0.161360-00 \\
0.173160-00\end{array}$ & $\begin{array}{l}0.496420 \\
0.523000 \\
0.549790 \\
0.576670 \\
0.603520\end{array}$ & $\begin{array}{l}02 \\
02 \\
02 \\
02 \\
02\end{array}$ & $\begin{array}{l}2.0032 \\
2.0034 \\
2.0036 \\
2.0038 \\
2.0040\end{array}$ & $\begin{array}{l}0.774970 \\
0.788850 \\
0.802220 \\
0.815070 \\
0.827380\end{array}$ & $\begin{array}{l}00 \\
00 \\
00 \\
00 \\
00\end{array}$ & $\begin{array}{l}0.706960 \\
0.681470 \\
0.655450 \\
0.629010 \\
0.602310\end{array}$ & $\begin{array}{l}02 \\
02 \\
02 \\
02 \\
02\end{array}$ \\
\hline $\begin{array}{l}1.9962 \\
1.9964 \\
1.9966 \\
1.9968 \\
1.9970\end{array}$ & $\begin{array}{l}0.18550 D-00 \\
0.198370-00 \\
0.211760-00 \\
0.225670-00 \\
0.240080-00\end{array}$ & $\begin{array}{l}0.630210 \\
0.656630 \\
0.682630 \\
0.708090 \\
0.732870\end{array}$ & $\begin{array}{l}02 \\
02 \\
02 \\
02 \\
02\end{array}$ & $\begin{array}{l}2.0042 \\
2.0044 \\
2.0046 \\
2.0048 \\
2.0050\end{array}$ & $\begin{array}{l}0.839160 \\
0.850400 \\
0.861100 \\
0.871270 \\
0.880910\end{array}$ & $\begin{array}{l}00 \\
00 \\
00 \\
00 \\
00\end{array}$ & $\begin{array}{l}0.575460 \\
0.548580 \\
0.521800 \\
0.495230 \\
0.468960\end{array}$ & $\begin{array}{l}02 \\
02 \\
02 \\
02 \\
02\end{array}$ \\
\hline $\begin{array}{l}1.9972 \\
1.9974 \\
1.9976 \\
1.9978 \\
1.9980\end{array}$ & $\begin{array}{l}0.254980-00 \\
0.270350-00 \\
0.286160-00 \\
0.302410-00 \\
0.319050-00\end{array}$ & $\begin{array}{l}0.756830 \\
0.779840 \\
0.801760 \\
0.822480 \\
0.841850\end{array}$ & $\begin{array}{l}02 \\
02 \\
02 \\
02 \\
02\end{array}$ & $\begin{array}{l}2.0052 \\
2.0054 \\
2.0057 \\
2.0060 \\
2.0065\end{array}$ & $\begin{array}{l}0.890030 \\
0.898640 \\
0.910620 \\
0.921510 \\
0.93738 D\end{array}$ & $\begin{array}{l}00 \\
00 \\
00 \\
00 \\
00\end{array}$ & $\begin{array}{l}0.443100 \\
0.417740 \\
0.380810 \\
0.345410 \\
0.290320\end{array}$ & $\begin{array}{l}02 \\
02 \\
02 \\
02 \\
02\end{array}$ \\
\hline $\begin{array}{l}1.9982 \\
1.9984 \\
1.9986 \\
1.9988 \\
1.9990\end{array}$ & $\begin{array}{l}0.336070-00 \\
0.353440-00 \\
0.371110-00 \\
0.389060-00 \\
0.407240-00\end{array}$ & $\begin{array}{l}0.859770 \\
0.876120 \\
0.890800 \\
0.903720 \\
0.914780\end{array}$ & $\begin{array}{l}02 \\
02 \\
02 \\
02 \\
02\end{array}$ & $\begin{array}{l}2.0070 \\
2.0075 \\
2.0080 \\
2.0085 \\
2.0090\end{array}$ & $\begin{array}{l}0.950630 \\
0.961540 \\
0.970400 \\
0.977490 \\
0.983100\end{array}$ & $\begin{array}{l}00 \\
00 \\
00 \\
00 \\
00\end{array}$ & $\begin{array}{l}0.240650 \\
0.196730 \\
0.158610 \\
0.126110 \\
0.988870\end{array}$ & $\begin{array}{l}02 \\
02 \\
02 \\
02 \\
01\end{array}$ \\
\hline $\begin{array}{l}1.9992 \\
1.9994 \\
1.9996 \\
1.9998 \\
2.0000\end{array}$ & $\begin{array}{l}0.42563 D-00 \\
0.44419 D-00 \\
0.46286 D-00 \\
0.481620-00 \\
0.50042000\end{array}$ & $\begin{array}{l}0.923930 \\
0.931100 \\
0.936240 \\
0.939320 \\
0.940310\end{array}$ & $\begin{array}{l}02 \\
02 \\
02 \\
02 \\
02\end{array}$ & $\begin{array}{l}2.0095 \\
2.0100 \\
2.0105 \\
2.0110\end{array}$ & $\begin{array}{l}0.987460 \\
0.990820 \\
0.993360 \\
0.995250\end{array}$ & $\begin{array}{l}00 \\
00 \\
00 \\
00\end{array}$ & $\begin{array}{l}0.764710 \\
0.583200 \\
0.438650 \\
0.325370\end{array}$ & $\begin{array}{ll}0 & 1 \\
0 & 1 \\
0 & 1 \\
0 & 1\end{array}$ \\
\hline
\end{tabular}


$E \mathbf{T A}=0.000010$

\begin{tabular}{|c|c|c|c|c|c|c|c|c|}
\hline Y & $\operatorname{PSI}(Y, E T A)$ & DPSI $(Y, E I A$ & A) /DY & Y & PSI I Y,E T & (A) & CPS I $(Y, E T$ & A) / DY \\
\hline $\begin{array}{l}1.9885 \\
1.9890 \\
1.9895 \\
1.9900 \\
1.9905\end{array}$ & $\begin{array}{l}0.507970-02 \\
0.697490-02 \\
0.946900-02 \\
0.127100-01 \\
0.168710-01\end{array}$ & $\begin{array}{l}0.327910 \\
0.434360 \\
0.568220 \\
0.734080 \\
0.936590\end{array}$ & $\begin{array}{ll}0 & 1 \\
0 & 1 \\
0 & 1 \\
01 \\
01 \\
01\end{array}$ & $\begin{array}{l}2.0002 \\
2.0004 \\
2.0006 \\
2.0008 \\
2.0010\end{array}$ & $\begin{array}{l}0.518280 \\
0.53608 D \\
0.553810 \\
0.571420 \\
0.58890 D\end{array}$ & $\begin{array}{l}00 \\
00 \\
00 \\
00 \\
00\end{array}$ & $\begin{array}{l}0.89112 \mathrm{C} \\
0.888410 \\
0.88394 \mathrm{D} \\
0.877730 \\
0.86982 \mathrm{D}\end{array}$ & $\begin{array}{l}02 \\
02 \\
02 \\
02 \\
02\end{array}$ \\
\hline $\begin{array}{l}1.9910 \\
1.9915 \\
1.9920 \\
1.9925 \\
1.9930\end{array}$ & $\begin{array}{l}0.221450-01 \\
0.28747 D-01 \\
0.36909 D-01 \\
0.46876 D-01 \\
0.58894 D-01\end{array}$ & $\begin{array}{l}0.118010 \\
0.146850 \\
0.180470 \\
0.219020 \\
0.262510\end{array}$ & $\begin{array}{l}02 \\
02 \\
02 \\
02 \\
02\end{array}$ & $\begin{array}{l}2.0012 \\
2.0014 \\
2.0016 \\
2.0018 \\
2.0020\end{array}$ & $\begin{array}{l}0.606210 \\
0.623300 \\
0.640160 \\
0.656750 \\
0.673040\end{array}$ & $\begin{array}{l}00 \\
00 \\
00 \\
00 \\
00\end{array}$ & $\begin{array}{l}0.860260 \\
0.849110 \\
0.836420 \\
0.822280 \\
0.806770\end{array}$ & $\begin{array}{l}02 \\
02 \\
02 \\
02 \\
02\end{array}$ \\
\hline $\begin{array}{l}1.9935 \\
1.9940 \\
1.9945 \\
1.9948 \\
1.9950\end{array}$ & $\begin{array}{l}0.732060-01 \\
0.90038 D-01 \\
0.10959 D-00 \\
0.122690-00 \\
0.132020-00\end{array}$ & $\begin{array}{l}0.310730 \\
0.363230 \\
0.419330 \\
0.454330 \\
0.478080\end{array}$ & $\begin{array}{l}02 \\
02 \\
02 \\
02 \\
02\end{array}$ & $\begin{array}{l}2.0022 \\
2.0024 \\
2.0026 \\
2.0028 \\
2.0030\end{array}$ & $\begin{array}{l}0.689010 \\
0.704630 \\
0.719880 \\
0.734740 \\
0.749190\end{array}$ & $\begin{array}{l}00 \\
00 \\
00 \\
00 \\
00\end{array}$ & $\begin{array}{l}0.78996 \mathrm{D} \\
0.771960 \\
0.752860 \\
0.732770 \\
0.711790\end{array}$ & $\begin{array}{l}02 \\
02 \\
02 \\
02 \\
02\end{array}$ \\
\hline $\begin{array}{l}1.9952 \\
1.9954 \\
1.9956 \\
1.9958 \\
1.9960\end{array}$ & $\begin{array}{l}0.141820-00 \\
0.152100-00 \\
0.162860-00 \\
0.174110-00 \\
0.185850-00\end{array}$ & $\begin{array}{l}0.502070 \\
0.526200 \\
0.550390 \\
0.574550 \\
0.598560\end{array}$ & $\begin{array}{l}02 \\
02 \\
02 \\
02 \\
02\end{array}$ & $\begin{array}{l}2.0032 \\
2.0034 \\
2.0036 \\
2.0038 \\
2.0040\end{array}$ & $\begin{array}{l}0.763210 \\
0.776780 \\
0.789910 \\
0.802570 \\
0.814750\end{array}$ & $\begin{array}{l}00 \\
00 \\
00 \\
00 \\
00\end{array}$ & $\begin{array}{l}0.690030 \\
0.667600 \\
0.644600 \\
0.621160 \\
0.597370\end{array}$ & $\begin{array}{l}02 \\
02 \\
02 \\
02 \\
02\end{array}$ \\
\hline $\begin{array}{l}1.9962 \\
1.9964 \\
1.9966 \\
1.9968 \\
1.9970\end{array}$ & $\begin{array}{l}0.198060-00 \\
0.210740-00 \\
0.223880-00 \\
0.237480-00 \\
0.251520-00\end{array}$ & $\begin{array}{l}0.622340 \\
0.645760 \\
0.668730 \\
0.691130 \\
0.712860\end{array}$ & $\begin{array}{l}02 \\
02 \\
02 \\
02 \\
02\end{array}$ & $\begin{array}{l}2.0042 \\
2.0044 \\
2.0046 \\
2.0048 \\
2.0050\end{array}$ & $\begin{array}{l}0.826460 \\
0.837680 \\
0.848430 \\
0.858680 \\
0.868460\end{array}$ & $\begin{array}{l}00 \\
00 \\
00 \\
00 \\
00\end{array}$ & $\begin{array}{l}0.573340 \\
0.549190 \\
0.524990 \\
0.500860 \\
0.476890\end{array}$ & $\begin{array}{l}02 \\
02 \\
02 \\
02 \\
02\end{array}$ \\
\hline $\begin{array}{l}1.9972 \\
1.9974 \\
1.9976 \\
1.9978 \\
1.9980\end{array}$ & $\begin{array}{l}0.265990-00 \\
0.280870-00 \\
0.296140-00 \\
0.311780-00 \\
0.327760-00\end{array}$ & $\begin{array}{l}0.733800 \\
0.753840 \\
0.772890 \\
0.790830 \\
0.807570\end{array}$ & $\begin{array}{l}02 \\
02 \\
02 \\
02 \\
02\end{array}$ & $\begin{array}{l}2.0052 \\
2.0054 \\
2.0057 \\
2.0060 \\
2.0065\end{array}$ & $\begin{array}{l}0.877760 \\
0.886590 \\
0.898960 \\
0.910320 \\
0.927100\end{array}$ & $\begin{array}{l}00 \\
00 \\
00 \\
00 \\
00\end{array}$ & $\begin{array}{l}0.453160 \\
0.429740 \\
0.395390 \\
0.362140 \\
0.309720\end{array}$ & $\begin{array}{l}02 \\
02 \\
02 \\
02 \\
02\end{array}$ \\
\hline $\begin{array}{l}1.9982 \\
1.9984 \\
1.9986 \\
1.9988 \\
1.9990\end{array}$ & $\begin{array}{l}0.344070-00 \\
0.360680-00 \\
0.377550-00 \\
0.394650-00 \\
0.411970-00\end{array}$ & $\begin{array}{l}0.823020 \\
0.837090 \\
0.849700 \\
0.860780 \\
0.870250\end{array}$ & $\begin{array}{l}02 \\
02 \\
02 \\
02 \\
02\end{array}$ & $\begin{array}{l}2.0070 \\
2.0075 \\
2.0080 \\
2.0085 \\
2.0090\end{array}$ & $\begin{array}{l}0.941370 \\
0.953340 \\
0.963270 \\
0.971400 \\
0.977970\end{array}$ & $\begin{array}{l}00 \\
00 \\
00 \\
00 \\
00\end{array}$ & $\begin{array}{l}0.261590 \\
0.218200 \\
0.179750 \\
0.146230 \\
0.117480\end{array}$ & $\begin{array}{l}02 \\
02 \\
02 \\
02 \\
02\end{array}$ \\
\hline $\begin{array}{l}1.9992 \\
1.9994 \\
1.9996 \\
1.9998 \\
2.0000\end{array}$ & $\begin{array}{l}0.429450-00 \\
0.447080-00 \\
0.464810-00 \\
0.482610-00 \\
0.50045000\end{array}$ & $\begin{array}{l}0.87808 D \\
0.884200 \\
0.888590 \\
0.891210 \\
0.89206 D\end{array}$ & $\begin{array}{l}02 \\
02 \\
02 \\
02 \\
02\end{array}$ & $\begin{array}{l}2.0095 \\
2.0100 \\
2.0105 \\
2.0110 \\
2.0115\end{array}$ & $\begin{array}{l}0.983220 \\
0.987360 \\
0.990590 \\
0.993070 \\
0.994950\end{array}$ & $\begin{array}{l}00 \\
00 \\
00 \\
00 \\
00\end{array}$ & $\begin{array}{l}0.932160 \\
0.730430 \\
0.565240 \\
0.431980 \\
0.326040\end{array}$ & $\begin{array}{ll}01 \\
01 \\
01 \\
01 \\
01\end{array}$ \\
\hline
\end{tabular}


$E T A=0.000020$

\begin{tabular}{|c|c|c|c|c|c|}
\hline Y & $P S I(Y, E T A)$ & DPSI $(Y, E T A) / D Y$ & $Y$ & $\operatorname{PSI}(Y, E T A)$ & DPSI $(Y, E T A) / D Y$ \\
\hline $\begin{array}{l}1.9825 \\
1.9850 \\
1.9875 \\
1.9900\end{array}$ & $\begin{array}{l}0.284260-02 \\
0.889110-02 \\
0.241430-01 \\
0.571050-01\end{array}$ & $\begin{array}{ll}0.137800 & 01 \\
0.380240 & 01 \\
0.897440 & 01 \\
0.181180 & 02\end{array}$ & $\begin{array}{l}2.0010 \\
2.0020 \\
2.0030 \\
2.0040 \\
2.0050\end{array}$ & $\begin{array}{ll}0.56344 D & 00 \\
0.624690 & 00 \\
0.68294 D & 00 \\
0.736970 & 00 \\
0.78586 D & 00\end{array}$ & $\begin{array}{ll}0.622790 & 02 \\
0.599720 & 02 \\
0.563240 & 02 \\
0.515920 & 02 \\
0.460910 & 02\end{array}$ \\
\hline $\begin{array}{l}1.9910 \\
1.9920 \\
1.9930 \\
1.9940 \\
1.9950\end{array}$ & $\begin{array}{l}0.775950-01 \\
0.103240-00 \\
0.134530-00 \\
0.171790-00 \\
0.215060-00\end{array}$ & $\begin{array}{ll}0.229690 & 02 \\
0.284000 & 02 \\
0.342480 & 02 \\
0.402810 & 02 \\
0.462070 & 02\end{array}$ & $\begin{array}{l}2.0060 \\
2.0070 \\
2.0080 \\
2.0090 \\
2.0100\end{array}$ & $\begin{array}{ll}0.829010 & 00 \\
0.866150 & 00 \\
0.897330 & 00 \\
0.922860 & 00 \\
0.94326 D & 00\end{array}$ & $\begin{array}{ll}0.401600 & 02 \\
0.341280 & 02 \\
0.282860 & 02 \\
0.228650 & 02 \\
0.180270 & 02\end{array}$ \\
\hline $\begin{array}{l}1.9960 \\
1.9970 \\
1.9980 \\
1.9990 \\
2.0000\end{array}$ & $\begin{array}{l}0.26406 D-00 \\
0.318190-00 \\
0.37652 D-00 \\
0.437810-00 \\
0.50063000\end{array}$ & $\begin{array}{ll}0.516960 & 02 \\
0.564090 & 02 \\
0.600320 & 02 \\
0.623100 & 02 \\
0.630780 & 02\end{array}$ & $\begin{array}{l}2.0110 \\
2.0125 \\
2.0150 \\
2.0175\end{array}$ & $\begin{array}{ll}0.959140 & 00 \\
0.97604 D & 00 \\
0.99118 D & 00 \\
0.997180 & 00\end{array}$ & $\begin{array}{ll}0.138620 & 02 \\
0.891850 & 01 \\
0.377400 & 01 \\
0.136600 & 01\end{array}$ \\
\hline
\end{tabular}

table for IHE EVALUATION OF THE FAXEN APPROXIMATION TO THE SOLUTICN OF THE LAMM EQUATICN

$\begin{array}{cl}Y & \text { PSI Y, ETA ) } \\ 1.9800 & 0.49394 D-02 \\ 1.9825 & 0.119950-01 \\ 1.9850 & 0.265230-01 \\ 1.9875 & 0.535030-01 \\ 1.9900 & 0.98690 D-01 \\ 1.9910 & 0.123030-00 \\ 1.9920 & 0.15130 D-00 \\ 1.9930 & 0.183590-00 \\ 1.9940 & 0.21986 D-00 \\ 1.9950 & 0.259930-00 \\ & \\ 1.9960 & 0.303470-00 \\ 1.9970 & 0.34999 D-00 \\ 1.9980 & 0.398870-00 \\ 1.9990 & 0.449410-00 \\ 2.0000 & 0.50077000\end{array}$

$E T A=0.000030$

\begin{tabular}{|c|c|c|}
\hline DPSI $I Y, E T A$ & A) / $/ D Y$ & Y \\
\hline $\begin{array}{l}0.184660 \\
0.403070 \\
0.792800 \\
0.140510 \\
0.224390\end{array}$ & $\begin{array}{l}01 \\
01 \\
01 \\
02 \\
02\end{array}$ & $\begin{array}{l}2.0010 \\
2.0020 \\
2.0030 \\
2.0040 \\
2.0050\end{array}$ \\
\hline $\begin{array}{l}0.262820 \\
0.302750 \\
0.342970 \\
0.382120 \\
0.418690\end{array}$ & $\begin{array}{l}02 \\
02 \\
02 \\
02 \\
02\end{array}$ & $\begin{array}{l}2.0060 \\
2.0070 \\
2.0080 \\
2.0090 \\
2.0100\end{array}$ \\
\hline $\begin{array}{l}0.451190 \\
0.478170 \\
0.498390 \\
0.510880 \\
0.515030\end{array}$ & $\begin{array}{l}02 \\
02 \\
02 \\
02 \\
02\end{array}$ & $\begin{array}{l}2.0110 \\
2.0125 \\
2.0150 \\
2.0175 \\
2.0200\end{array}$ \\
\hline
\end{tabular}

DPSI $(Y, E T A) / D Y$

0.51063002 0.49790002 $0.477460 \quad 02$ 0.45029002 0.41765002

$0.380970 \quad 02$ $0.341770 \quad 02$ $0.301540 \quad 02$ 0.26164002 0.22327002

$0.187380 \quad 02$ 0.13963002 0.78687001 0.39956001 $0.18282 \mathrm{C} 01$ 
$E T A=0.000040$

Y

PSI $(Y, E T A)$

DPSI ( Y,ETA ) /DY

0.18953001

0.36797001

0.66070001

0.16850002

0.20995002

0.23934002

0.26945002

0.29958002

0.32894002

$0.356700 \quad 02$

0.38199002

0.40399002

0.42195002

0.43523002

0.44336002

0.44603002

$0.412400-00$

$0.456380-00$

0.50089000
$P S I(Y, E T A)$

$0.54540 \mathrm{D} 00$

0.58934000

0.63218000

0.67345000

0.71269000

0.74954000

0.78373000

0.81505000

0.84338000

0.86870000

0.89104000

2.0110

2.0125

2.0150

2.0175

2.0200

2.0225

0.97493000

0.98740000

0.99410000
DPSI (Y, ETA )/DY

$0.443140 \quad 02$ 0.43480002 0.42132002 0.40318002

0.38103002

$0.355630 \quad 02$ $0.327790 \quad 02$

0.29838002

0.26824002

0.23815002

0.20880002

$0.167450 \quad 02$

$0.108900 \quad 02$

$0.654940 \quad 01$

$0.364310 \quad 01$

0.18741001

1.9970

TABLE FOR THE EVALUATION OF THE FAXEN APPROXIMATION TO THE SOLUTION OF THE LAMM EQUATION

$E T A=0.000050$

r

PSI $(Y, E T A)$

$0.625390-02$

$0.123040-01$

$0.228860-01$

$0.402760-01$

$0.671330-01$

$0.889110-01$

$0.115560-00$

$0.136210-00$

$0.15926 \mathrm{D}-00$

$0.184730-00$

$0.212580-00$

$0.242750-00$

$0.275090-00$

$0.309420-00$

$0.345500-00$

$0.38304 \mathrm{D}-00$

$0.421720-00$

$0.461160-00$

0.50100000
DPSI $(Y, E T A) / D Y$

0.17639001

0.31919001

0.54262001

0.86656001

0.13001002

0.16093002

0.19477002

0.21845002

0.24258002

0.26668002

0.29027002

0.31280002

0.33372002

0.35250002

0.36864002

$0.381670 \quad 02$

0.39123002

$0.397050 \quad 02$

0.39894002
$\operatorname{PSI}(Y, E T A)$

0.54082000

0.58024000

$0.61886 \mathrm{D} 00$

0.65634000

0.69234000

0.72658000

0.75881000

$0.78887 \mathrm{D} 00$

0.81660000

0.84195000

0.86488000

0.88541000

0.90363000

0.91962000

0.93351000

0.96016000

0.97738000

0.98785000

0.99383000
DPSI (Y, ETA )/DY

0.39685002

$0.39084 \mathrm{D} 02$

0.38110002

$0.367900 \quad 02$

0.35162002

$0.332720 \quad 02$

0.31171002

0.28911002

0.26549002

0.24137002

$0.217250 \quad 02$

0.19360002

0.17081002

$0.149200 \quad 02$

$0.129030 \quad 02$

0.85901001

0.53722001

0.31562001

0.17420001 
$E T A=0.000060$

\begin{tabular}{|c|c|c|c|c|c|c|c|c|}
\hline$Y$ & $P S I(Y, E T A)$ & DPSI $(Y, E T A$ & A) /OY & & PSI $(Y, E T$ & (A) & CPSII Y, ETA & A) /OY \\
\hline 1.9725 & $0.607710-02$ & 0.156980 & 01 & $\begin{array}{l}2.0010 \\
2.0020 \\
2.0030 \\
2.0040 \\
2.0050\end{array}$ & $\begin{array}{l}0.537460 \\
0.573510 \\
0.608960 \\
0.643520 \\
0.67694 D\end{array}$ & $\begin{array}{l}00 \\
00 \\
00 \\
00 \\
00\end{array}$ & $\begin{array}{l}0.362570 \\
0.357980 \\
0.350510 \\
0.340350 \\
0.327740\end{array}$ & $\begin{array}{l}02 \\
02 \\
02 \\
02 \\
02\end{array}$ \\
\hline $\begin{array}{l}1.9750 \\
1.9775 \\
1.9800 \\
1.9825 \\
1.9850\end{array}$ & $\begin{array}{l}0.11321 \mathrm{D}-01 \\
0.20123 \mathrm{D}-01 \\
0.34152 \mathrm{D}-01 \\
0.55381 \mathrm{D}-01 \\
0.85882 \mathrm{D}-01\end{array}$ & $\begin{array}{l}0.271060 \\
0.444300 \\
0.691310 \\
0.102100 \\
0.143150\end{array}$ & $\begin{array}{l}01 \\
01 \\
01 \\
02 \\
02\end{array}$ & $\begin{array}{l}2.0060 \\
2.0070 \\
2.0080 \\
2.0090 \\
2.0100\end{array}$ & $\begin{array}{l}0.709000 \\
0.739480 \\
0.768230 \\
0.795120 \\
0.820060\end{array}$ & $\begin{array}{l}00 \\
00 \\
00 \\
00 \\
00\end{array}$ & $\begin{array}{l}0.312980 \\
0.296410 \\
0.278380 \\
0.259280 \\
0.239480\end{array}$ & $\begin{array}{l}02 \\
02 \\
02 \\
02 \\
02\end{array}$ \\
\hline $\begin{array}{l}1.9860 \\
1.9870 \\
1.9880 \\
1.9890 \\
1.9900\end{array}$ & $\begin{array}{l}0.101110-00 \\
0.11821 D-00 \\
0.137260-00 \\
0.158310-00 \\
0.181380-00\end{array}$ & $\begin{array}{l}0.161500 \\
0.180680 \\
0.200470 \\
0.220570 \\
0.240680\end{array}$ & $\begin{array}{l}02 \\
02 \\
02 \\
02 \\
02\end{array}$ & $\begin{array}{l}2.0110 \\
2.0120 \\
2.0130 \\
2.0140 \\
2.0150\end{array}$ & $\begin{array}{l}0.84301 D \\
0.86394 D \\
0.88287 D \\
0.89986 D \\
0.91497 D\end{array}$ & $\begin{array}{l}00 \\
00 \\
00 \\
00 \\
00\end{array}$ & $\begin{array}{l}0.219360 \\
0.199270 \\
0.179510 \\
0.160370 \\
0.142080\end{array}$ & $\begin{array}{l}02 \\
02 \\
02 \\
02 \\
02\end{array}$ \\
\hline $\begin{array}{l}1.9910 \\
1.9920 \\
1.9930 \\
1.9940 \\
1.9950\end{array}$ & $\begin{array}{l}0.20644 D-00 \\
0.23344 D-00 \\
0.262300-00 \\
0.292880-00 \\
0.325030-00\end{array}$ & $\begin{array}{l}0.260450 \\
0.279490 \\
0.297450 \\
0.313920 \\
0.328560\end{array}$ & $\begin{array}{l}02 \\
02 \\
02 \\
02 \\
02\end{array}$ & $\begin{array}{l}2.0160 \\
2.0175 \\
2.0200 \\
2.0225 \\
2.0250\end{array}$ & $\begin{array}{l}0.928310 \\
0.945230 \\
0.966260 \\
0.980140 \\
0.988840\end{array}$ & $\begin{array}{l}00 \\
00 \\
00 \\
00 \\
00\end{array}$ & $\begin{array}{l}0.124840 \\
0.101220 \\
0.684430 \\
0.4339330 \\
0.267700\end{array}$ & $\begin{array}{l}02 \\
02 \\
01 \\
01 \\
01\end{array}$ \\
\hline $\begin{array}{l}1.9960 \\
1.9970 \\
1.9980 \\
1.9990 \\
2.0000\end{array}$ & $\begin{array}{l}0.35852 D-00 \\
0.39315 D-00 \\
0.42864 D-00 \\
0.46472 D-00 \\
0.50109 D 00\end{array}$ & $\begin{array}{l}0.341030 \\
0.35104 D \\
0.35834 D \\
0.36276 D \\
0.36418 D\end{array}$ & $\begin{array}{l}02 \\
02 \\
02 \\
02 \\
02\end{array}$ & 2.0275 & 0.994020 & 00 & $0.15484 \mathrm{D}$ & 01 \\
\hline
\end{tabular}

IABLE FOR THE EVALUATION OF THE FAXEN APPROXIMATION TO THE SOLUTICN OF THE LAMM EQUATICN

$$
E T A=0.000070
$$

Y

PSI (Y, ETA)

$0.566290-02$

$0.101380-01$

$0.174330-01$

$0.288070-01$

$0.457690-01$

$0.699650-01$

$0.102980-00$

1.9860

1.9870

1.9880

1.9890

1.9900

1.9910

1.9920

1.9930

1.9940

1. 9950

1.9960

1.9970

1.9780

1.9990

2.0000

$0.11895 \mathrm{D}-00$

$0.136600-00$

$0.155960-00$

$0.177040-00$

$0.199840-00$

$0.22432 D-00$

$0.25042 D-00$

$0.278050-00$

$0.307090-00$

$0.337380-00$

$0.368770-00$

$0.434050-00$

$0.46750 D-00$

0.50118000
DPSI (Y,ETA)/DY

0.13651001

0.22796001

0.36405001

0.55600001

0.81209001

$0.113430 \quad 02$

0.15153002

$0.401070-00$
$0.16802 D 02$ 0.18498002 0.20221002

0.21946002 0.23650002

0.25304002 0.26881002 0.28353002 0.29693002 $0.308750 \quad 02$

$0.318760 \quad 02$ 0.32674002 0.33255002 $0.336050 \quad 02$ 0.33716002
PSI ( $Y, E T A)$

0.53485000 $0.568280 \quad 00$ $0.601220 \quad 00$ 0.63345000 0.66478000

2. 0020

2.0030

2.0040

2.0050

2. 0060

2.0070

2.0080

2.0090

2.0100

2.0110

2.0120

2. 0130

2.0140

2.0150

2.0160

2. 0175

2. 0200

2. 0225

2.0250

2.0275

2.0300
0.69499000 0.72393000 0.75146000 $0.777440 \quad 00$ 0.80181000

$0.824490 \quad 00$ 0.84546000 0.86469000 0.88222000 $0.89808 D \quad 00$

0.91232000 0.93083000 $0.954800 \quad 00$ $0.982820 \quad 00$

0.99002000 0.99443000 $0.971580 \quad 00$
DPSI (Y,ETA)/DY

$0.335880 \quad 02$ $0.332210 \quad 02$ $0.326250 \quad 02$ $0.318120 \quad 02$ 0.30798002

$0.296040 \quad 02$ $0.282540 \quad 02$ $0.267740 \quad 02$ 0.25190002 0.23532002

0.21826002 0.20100002 0.18378002

$0.166850 \quad 02$ 0.15039002

0.13460002 $0.112440 \quad 02$ 0.80401001 $0.549780 \quad 01$ $0.359530 \quad 01$

$0.224850 \quad 01$ 0.13448001 
$E T A=0.000080$

\begin{tabular}{|c|c|c|c|c|c|c|c|c|}
\hline$Y$ & $P S I(Y, E T A)$ & DPSI $Y, E T A$ & A) /DY & $Y$ & PS I $(Y, E I$ & (A) & DPS I $(Y, E T$ & A) / D \\
\hline $\begin{array}{l}1.9675 \\
1.9700 \\
1.9725 \\
1.9750\end{array}$ & $\begin{array}{l}0.514150-02 \\
0.892960-02 \\
0.149700-01 \\
0.242340-01\end{array}$ & $\begin{array}{l}0.117190 \\
0.190840 \\
0.298870 \\
0.450130\end{array}$ & $\begin{array}{l}01 \\
01 \\
01 \\
01\end{array}$ & $\begin{array}{l}2.0010 \\
2.0020 \\
2.0030 \\
2.0040 \\
2.0050\end{array}$ & $\begin{array}{l}0.532760 \\
0.564060 \\
0.594960 \\
0.625280 \\
0.654850\end{array}$ & $\begin{array}{l}00 \\
00 \\
00 \\
00 \\
00\end{array}$ & $\begin{array}{l}0.314320 \\
0.311310 \\
0.306410 \\
0.299710 \\
0.291320\end{array}$ & $\begin{array}{l}02 \\
02 \\
02 \\
02 \\
02\end{array}$ \\
\hline $\begin{array}{l}1.9775 \\
1.9800 \\
1.9825 \\
1.9840 \\
1.9850\end{array}$ & $\begin{array}{l}0.378990-01 \\
0.572870-01 \\
0.837440-01 \\
0.10352 D-00 \\
0.118470-00\end{array}$ & $\begin{array}{l}0.651980 \\
0.908150 \\
0.121650 \\
0.142280 \\
0.156720\end{array}$ & $\begin{array}{l}01 \\
01 \\
02 \\
02 \\
02\end{array}$ & $\begin{array}{l}2.0060 \\
2.0070 \\
2.0080 \\
2.0090 \\
2.0100\end{array}$ & $\begin{array}{l}0.683500 \\
0.711080 \\
0.737490 \\
0.762590 \\
0.786320\end{array}$ & $\begin{array}{l}00 \\
00 \\
00 \\
00 \\
00\end{array}$ & $\begin{array}{l}0.281410 \\
0.270140 \\
0.257700 \\
0.244310 \\
0.230170\end{array}$ & $\begin{array}{l}02 \\
02 \\
02 \\
02 \\
02\end{array}$ \\
\hline $\begin{array}{l}1.9860 \\
1.9870 \\
1.9880 \\
1.9890 \\
1.9900\end{array}$ & $\begin{array}{l}0.134880-00 \\
0.152780-00 \\
0.172200-00 \\
0.193120-00 \\
0.215520-00\end{array}$ & $\begin{array}{l}0.171540 \\
0.186590 \\
0.201710 \\
0.216680 \\
0.231320\end{array}$ & $\begin{array}{l}02 \\
02 \\
02 \\
02 \\
02\end{array}$ & $\begin{array}{l}2.0110 \\
2.0120 \\
2.0130 \\
2.0140 \\
2.0150\end{array}$ & $\begin{array}{l}0.808610 \\
0.829410 \\
0.848700 \\
0.866490 \\
0.88278 D\end{array}$ & $\begin{array}{l}00 \\
00 \\
00 \\
00 \\
00\end{array}$ & $\begin{array}{l}0.215490 \\
0.200500 \\
0.185390 \\
0.170340 \\
0.155550\end{array}$ & $\begin{array}{l}02 \\
02 \\
02 \\
02 \\
02\end{array}$ \\
\hline $\begin{array}{l}1.9910 \\
1.9920 \\
1.9930 \\
1.9940 \\
1.9950\end{array}$ & $\begin{array}{l}0.239370-00 \\
0.264580-00 \\
0.291080-00 \\
0.31876 D-00 \\
0.347490-00\end{array}$ & $\begin{array}{l}0.245410 \\
0.258730 \\
0.271080 \\
0.282250 \\
0.292050\end{array}$ & $\begin{array}{l}02 \\
02 \\
02 \\
02 \\
02\end{array}$ & $\begin{array}{l}2.0160 \\
2.0170 \\
2.0185 \\
2.0200 \\
2.0225\end{array}$ & $\begin{array}{l}0.897610 \\
0.911030 \\
0.928640 \\
0.94344 D \\
0.96262 D\end{array}$ & $\begin{array}{l}00 \\
00 \\
00 \\
00 \\
00\end{array}$ & $\begin{array}{l}0.141150 \\
0.127290 \\
0.107730 \\
0.899120 \\
0.644690\end{array}$ & $\begin{array}{l}02 \\
02 \\
02 \\
01 \\
01\end{array}$ \\
\hline $\begin{array}{l}1.9960 \\
1.9970 \\
1.9980 \\
1.9990 \\
2.0000\end{array}$ & $\begin{array}{l}0.377120-00 \\
0.407490-00 \\
0.438430-00 \\
0.469750-00 \\
0.50126000\end{array}$ & $\begin{array}{l}0.300310 \\
0.306870 \\
0.311620 \\
0.314480 \\
0.315390\end{array}$ & $\begin{array}{l}02 \\
02 \\
02 \\
02 \\
02\end{array}$ & $\begin{array}{l}2.0250 \\
2.0275 \\
2.0300 \\
2.0325\end{array}$ & $\begin{array}{l}0.976120 \\
0.985270 \\
0.991220 \\
0.994950\end{array}$ & $\begin{array}{l}00 \\
00 \\
00 \\
00\end{array}$ & $\begin{array}{l}0.444550 \\
0.294790 \\
0.188000 \\
0.115300\end{array}$ & $\begin{array}{ll}0 & 1 \\
0 & 1 \\
0 & 1 \\
0 & 1\end{array}$ \\
\hline
\end{tabular}

TABLE FOR THE EVALUATION OF THE FAXEN APPROXIMATION TO THE SOLUTION OF THE LAMM EQUATICN

$E T A=0.000090$

Y

\begin{abstract}
PSI (Y,ETA)
\end{abstract}
DPSI (Y, ETA ) /DY

$\begin{array}{ll}1.9650 & 0.45888 D-02 \\ 1.9675 & 0.77811 D-02 \\ 1.9700 & 0.12785 D-01 \\ 1.9725 & 0.20361 D-01 \\ 1.9750 & 0.31442 D-01 \\ 1.9775 & 0.47097 D-01 \\ 1.9800 & 0.68462 D-01 \\ & \\ 1.9810 & 0.78856 D-01 \\ 1.9820 & 0.90404 D-01 \\ 1.9830 & 0.10316 D-00 \\ 1.9840 & 0.11718 D-00 \\ 1.9850 & 0.13250 D-00 \\ 1.9860 & 0.14914 D-00 \\ 1.9870 & 0.16712 D-00 \\ 1.9880 & 0.18645 D-00 \\ 1.9890 & 0.20710 D-00 \\ 1.9900 & 0.229050-00 \\ & \\ 1.9910 & 0.25224 D-00 \\ 1.9920 & 0.276620-00 \\ 1.9930 & 0.30209 D-00 \\ 1.9940 & 0.32857 D-00 \\ 1.9950 & 0.35594 D-00 \\ 1.9960 & 0.38408 D-00 \\ 1.9970 & 0.41284 D-00 \\ 1.9980 & 0.44207 D-00 \\ 1.9990 & 0.47163 D-00 \\ 2.0000 & 0.50134 D 00 \\ & \end{array}$

\begin{tabular}{|c|c|}
\hline $\begin{array}{l}0.998370 \\
0.159440\end{array}$ & $\begin{array}{l}00 \\
01\end{array}$ \\
\hline $\begin{array}{l}0.245930 \\
0.366400 \\
0.527250 \\
0.732820 \\
0.983780\end{array}$ & $\begin{array}{ll}0 & 1 \\
01 \\
01 \\
01 \\
01\end{array}$ \\
\hline $\begin{array}{l}0.109610 \\
0.121440 \\
0.133810 \\
0.146620 \\
0.159760\end{array}$ & $\begin{array}{l}02 \\
02 \\
02 \\
02 \\
02\end{array}$ \\
\hline $\begin{array}{l}0.173120 \\
0.186550 \\
0.199920 \\
0.213060 \\
0.225800\end{array}$ & $\begin{array}{l}02 \\
02 \\
02 \\
02 \\
02\end{array}$ \\
\hline $\begin{array}{l}0.237970 \\
0.249420 \\
0.259970 \\
0.269460 \\
0.277750\end{array}$ & $\begin{array}{l}02 \\
02 \\
02 \\
02 \\
02\end{array}$ \\
\hline $\begin{array}{l}0.284710 \\
0.290230 \\
0.294210 \\
0.296600 \\
0.297350\end{array}$ & $\begin{array}{l}02 \\
02 \\
02 \\
02 \\
02\end{array}$ \\
\hline
\end{tabular}

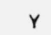

2. 0010

2.0020

2.0030

2.0040

2.0050

2.0060

2.0070

2.0080

2.0090

2.0100

2.0110

2.0120

2.0130

2.0140

2.0150

2.0160

2.0170

2.0180

2.0190

2.0200

2.0210

2.0225

2.0250

2.0275

2.0300

2.0325

2.0350
PSI (Y,ETA)

0.53104000 0.56057000 0.58977000 0.61848000 0.64655000

0.67385000 0.70024000 $0.72562 \mathrm{D} 00$ $0.74990 D 00$ 0.77298000

0.79481000 0.81535000 $0.834550 \quad 00$ $0.852410 \quad 00$ $0.868940 \quad 00$

0.88413000 $0.898040 \quad 00$ 0.91068000 $0.922130 \quad 00$ $0.932420 \quad 00$

$0.941630 \quad 00$ 0.95356000 0.96903000 0.97997000 $0.987430 \quad 00$

0.99236000 0.99550000
DPSI (Y,EIA ) / DY

0.29645002 0.29392002 0.28979002 0.28414002 0.27705002

$0.268650 \quad 02$ 0.25906002 $0.248420 \quad 02$ 0.23691002 0.22467002

0.21189002 0.19872002 0.18535002 0.17191002 0.15857002

$0.145450 \quad 02$ 0.13267002 0.12035002 0.10857002 0.97400001

$0.868930 \quad 01$ 0.72462001 $0.520700 \quad 01$ 0.36139001 0.24227001

0.15687001 0.98105000 
$E T A=0.000100$

\begin{tabular}{|c|c|c|c|c|c|c|c|}
\hline Y & PSI $(Y, E T A)$ & DPSI $(Y, E T A) / D Y$ & Y & PSI $I Y, E$ & TA) & DPSII $Y, E T$ & A) $/ D Y$ \\
\hline $\begin{array}{l}1.9625 \\
1.9650 \\
1.9675\end{array}$ & $\begin{array}{l}0.404750-02 \\
0.673100-02 \\
0.108800-01\end{array}$ & $\begin{array}{ll}0.846600 & 00 \\
0.133100 & 01 \\
0.202830 & 01\end{array}$ & $\begin{array}{l}2.0010 \\
2.0020 \\
2.0030 \\
2.0040 \\
2.0050\end{array}$ & $\begin{array}{l}0.529590 \\
0.557630 \\
0.585380 \\
0.612700 \\
0.639490\end{array}$ & $\begin{array}{l}00 \\
00 \\
00 \\
00 \\
00\end{array}$ & $\begin{array}{l}0.281310 \\
0.279140 \\
0.275610 \\
0.270760 \\
0.264670\end{array}$ & $\begin{array}{l}02 \\
02 \\
02 \\
02 \\
02\end{array}$ \\
\hline $\begin{array}{l}1.9700 \\
1.9725 \\
1.9750 \\
1.9775 \\
1.9800\end{array}$ & $\begin{array}{l}0.170980-01 \\
0.261300-01 \\
0.388480-01 \\
0.562070-01 \\
0.791720-01\end{array}$ & $\begin{array}{ll}0.299570 & 01 \\
0.428850 & 01 \\
0.595020 & 01 \\
0.800180 & 01 \\
0.104300 & 02\end{array}$ & $\begin{array}{l}2.0060 \\
2.0070 \\
2.0080 \\
2.0090 \\
2.0100\end{array}$ & $\begin{array}{l}0.665600 \\
0.690940 \\
0.715390 \\
0.738890 \\
0.761340\end{array}$ & $\begin{array}{l}00 \\
00 \\
00 \\
00 \\
00\end{array}$ & $\begin{array}{l}0.257420 \\
0.249130 \\
0.239900 \\
0.229860 \\
0.219140\end{array}$ & $\begin{array}{l}02 \\
02 \\
02 \\
02 \\
02\end{array}$ \\
\hline $\begin{array}{l}1.9810 \\
1.9820 \\
1.9830 \\
1.9840 \\
1.9850\end{array}$ & $\begin{array}{l}0.901310-01 \\
0.102180-00 \\
0.115360-00 \\
0.129700-00 \\
0.145230-00\end{array}$ & $\begin{array}{ll}0.114950 & 02 \\
0.126060 & 02 \\
0.137550 & 02 \\
0.149340 & 02 \\
0.161340 & 02\end{array}$ & $\begin{array}{l}2.0110 \\
2.0120 \\
2.0130 \\
2.0140 \\
2.0150\end{array}$ & $\begin{array}{l}0.782700 \\
0.802910 \\
0.821930 \\
0.839760 \\
0.856380\end{array}$ & $\begin{array}{l}00 \\
00 \\
00 \\
00 \\
00\end{array}$ & $\begin{array}{l}0.207880 \\
0.196220 \\
0.184290 \\
0.172210 \\
0.160130\end{array}$ & $\begin{array}{l}02 \\
02 \\
02 \\
02 \\
02\end{array}$ \\
\hline $\begin{array}{l}1.9860 \\
1.9870 \\
1.9880 \\
1.9890 \\
1.9900\end{array}$ & $\begin{array}{l}0.161970-00 \\
0.179910-00 \\
0.199060-00 \\
0.219380-00 \\
0.240850-00\end{array}$ & $\begin{array}{ll}0.173420 & 02 \\
0.185490 & 02 \\
0.197400 & 02 \\
0.209030 & 02 \\
0.220240 & 02\end{array}$ & $\begin{array}{l}2.0160 \\
2.0170 \\
2.0180 \\
2.0190 \\
2.0200\end{array}$ & $\begin{array}{l}0.871790 \\
0.886010 \\
0.899080 \\
0.911010 \\
0.921870\end{array}$ & $\begin{array}{l}00 \\
00 \\
00 \\
00 \\
00\end{array}$ & $\begin{array}{l}0.148150 \\
0.136390 \\
0.124930 \\
0.113860 \\
0.103260\end{array}$ & $\begin{array}{l}02 \\
02 \\
02 \\
02 \\
02\end{array}$ \\
\hline $\begin{array}{l}1.9910 \\
1.9920 \\
1.9930 \\
1.9940 \\
1.9950\end{array}$ & $\begin{array}{l}0.263410-00 \\
0.287010-00 \\
0.311560-00 \\
0.336980-00 \\
0.363160-00\end{array}$ & $\begin{array}{ll}0.230900 & 02 \\
0.240860 & 02 \\
0.250000 & 02 \\
0.258200 & 02 \\
0.265330 & 02\end{array}$ & $\begin{array}{l}2.0210 \\
2.0225 \\
2.0250 \\
2.0275 \\
2.0300\end{array}$ & $\begin{array}{l}0.93168 D \\
0.94459 D \\
0.96174 D \\
0.97430 D \\
0.98320 D\end{array}$ & $\begin{array}{l}00 \\
00 \\
00 \\
00 \\
00\end{array}$ & $\begin{array}{l}0.931770 \\
0.791230 \\
0.587630 \\
0.422990 \\
0.295120\end{array}$ & $\begin{array}{ll}01 \\
01 \\
01 \\
01 \\
01\end{array}$ \\
\hline $\begin{array}{l}1.9960 \\
1.9970 \\
1.9980 \\
1.9990 \\
2.0000\end{array}$ & $\begin{array}{l}0.390010-00 \\
0.417380-00 \\
0.445170-00 \\
0.473220-00 \\
0.50141000\end{array}$ & $\begin{array}{ll}0.271300 & 02 \\
0.276020 & 02 \\
0.279420 & 02 \\
0.281460 & 02 \\
0.282090 & 02\end{array}$ & $\begin{array}{l}2.0325 \\
2.0350 \\
2.0375\end{array}$ & $\begin{array}{l}0.98932 D \\
0.99340 D \\
0.99604 D\end{array}$ & $\begin{array}{l}00 \\
00 \\
00\end{array}$ & $\begin{array}{l}0.199560 \\
0.130800 \\
0.830880\end{array}$ & $\begin{array}{l}01 \\
01 \\
00\end{array}$ \\
\hline
\end{tabular}

TABLE FOR THE EVALUATION OF THE FAXEN APPROXIMATION TO THE SOLUTICN OF THE LAMM EQUATICN

$E T A=0.000200$

PS I (Y, ETA)

$0.629900-02$

1.9503

1.9550

1.9600

1.9650

1.9700

1.9730

1.9760

1.9780

1.9800

1.9823

1.9840

1. 9860

1.9880

1.9700

1.9923

$1.994 J$

1.9963

1.9980

2.0000
$0.123860-01$

$0.230240-01$

$0.404960-01$

$0.674620-01$

$0.893180-01$

$0.116050-00$

$0.136760-00$

$0.159870-00$

$0.185400-00$

$0.213310-00$

$0.243530-00$

$0.275930-00$

$0.310300-00$

$0.346430-00$

$0.384000-00$

$0.422700-00$

$0.462160-00$

0.50199000
DPSI (Y, ETA ) /DY

$0.887540 \quad 00$

$0.160510 \mathrm{Cl}$

0.27268001

0.43519001

0.65248001

0.80735001

0.97677001

$0.109530 \quad 02$

0.12159002

$0.133640 \quad 02$

0.14542002

$0.156670 \quad 02$

0.16711002

$0.176470 \quad 02$

$0.184500 \quad 02$

0.19097002

0.19571002

$0.198570 \quad 02$

0.19946002
PSI $Y$, ETA)

0.54181000 0.58121000 0.61981000 0.65726000 0.69322000

0.72741000 $0.759590 \quad 00$ 0.78958000 $0.817260 \quad 00$ 0.84255000

0.86541000 0.88589000 0.90405000 0.91998000 0.93383000

0.96037000 0.97752000 0.98793000 0.99388000
DPSI $(Y, E T A) / D Y$

0.19837002 0.19532002 $0.190400 \quad 02$ $0.183760 \quad 02$ 0.17559002

0.16611002 $0.155580 \quad 02$ 0.14426002 $0.13244 \mathrm{D} \quad 02$ 0.12038002

0.10833002 0.96512001 0.85130001 $0.74342 \mathrm{C} \mathrm{OI}$ $0.642760 \quad 01$

0.42765001 0.26729001 $0.156940 \quad 01$ 0.86563000 
$E T A=0.000300$

\begin{tabular}{|c|c|c|c|c|c|c|c|}
\hline$Y$ & $P S I(Y, E T A)$ & DPSI $(Y, E T A) / D Y$ & $Y$ & PSI $(Y, E T$ & (A) & DPSIIY, ETA & A) $/ D Y$ \\
\hline $\begin{array}{l}1.9350 \\
1.9400 \\
1.9450\end{array}$ & $\begin{array}{l}0.405580-02 \\
0.727740-02 \\
0.125730-01\end{array}$ & $\begin{array}{l}0.489670-00 \\
0.82326000 \\
0.13276001\end{array}$ & $\begin{array}{l}2.0020 \\
2.0040 \\
2.0060 \\
2.0080 \\
2.0100\end{array}$ & $\begin{array}{l}0.534970 \\
0.567270 \\
0.599120 \\
0.630320 \\
0.660690\end{array}$ & $\begin{array}{l}00 \\
00 \\
00 \\
00 \\
00\end{array}$ & $\begin{array}{l}0.162240 \\
0.160540 \\
0.157810 \\
0.154090 \\
0.149460\end{array}$ & $\begin{array}{l}02 \\
02 \\
02 \\
02 \\
02\end{array}$ \\
\hline $\begin{array}{l}1.9500 \\
1.9550 \\
1.9600 \\
1.9650 \\
1.9700\end{array}$ & $\begin{array}{l}0.209230-01 \\
0.33556 D-01 \\
0.51889 D-01 \\
0.77413 D-01 \\
0.11150 D-00\end{array}$ & $\begin{array}{ll}0.205360 & 01 \\
0.304700 & 01 \\
0.433650 & 01 \\
0.591970 & 01 \\
0.775120 & 01\end{array}$ & $\begin{array}{l}2.0120 \\
2.0140 \\
2.0160 \\
2.0180 \\
2.0200\end{array}$ & $\begin{array}{l}0.690050 \\
0.718250 \\
0.745150 \\
0.770640 \\
0.794630\end{array}$ & $\begin{array}{l}00 \\
00 \\
00 \\
00 \\
00\end{array}$ & $\begin{array}{l}0.144010 \\
0.137840 \\
0.131050 \\
0.123770 \\
0.116110\end{array}$ & $\begin{array}{l}02 \\
02 \\
02 \\
02 \\
02\end{array}$ \\
\hline $\begin{array}{l}1.9720 \\
1.9740 \\
1.9760 \\
1.9780 \\
1.9800\end{array}$ & $\begin{array}{l}0.12778 D-00 \\
0.145650-00 \\
0.16512 D-00 \\
0.18620 D-00 \\
0.20887 D-00\end{array}$ & $\begin{array}{ll}0.853360 & 01 \\
0.933250 & 01 \\
0.101380 & 02 \\
0.109410 & 02 \\
0.117280 .02\end{array}$ & $\begin{array}{l}2.0220 \\
2.0240 \\
2.0260 \\
2.0280 \\
2.0300\end{array}$ & $\begin{array}{l}0.817070 \\
0.837900 \\
0.857130 \\
0.874760 \\
0.890810\end{array}$ & $\begin{array}{l}00 \\
00 \\
00 \\
00 \\
00\end{array}$ & $\begin{array}{l}0.108210 \\
0.100170 \\
0.921200 \\
0.841500 \\
0.763580\end{array}$ & $\begin{array}{l}02 \\
02 \\
01 \\
01 \\
01\end{array}$ \\
\hline $\begin{array}{l}1.9820 \\
1.9840 \\
1.9860 \\
1.9880 \\
1.9900\end{array}$ & $\begin{array}{l}0.233090-00 \\
0.25880 D-00 \\
0.28590 D-00 \\
0.31428 D-00 \\
0.34380 D-00\end{array}$ & $\begin{array}{ll}0.124890 & 02 \\
0.132100 & 02 \\
0.138800 & 02 \\
0.144880 & 02 \\
0.150210 & 02\end{array}$ & $\begin{array}{l}2.0320 \\
2.0350 \\
2.0400 \\
2.0450 \\
2.0500\end{array}$ & $\begin{array}{l}0.905320 \\
0.924350 \\
0.949400 \\
0.967350 \\
0.979690\end{array}$ & $\begin{array}{l}00 \\
00 \\
00 \\
00 \\
00\end{array}$ & $\begin{array}{l}0.688280 \\
0.581700 \\
0.425060 \\
0.297920 \\
0.200290\end{array}$ & $\begin{array}{ll}01 \\
01 \\
01 \\
01 \\
01\end{array}$ \\
\hline $\begin{array}{l}1.9920 \\
1.9940 \\
1.9960 \\
1.9980 \\
2.0000\end{array}$ & $\begin{array}{l}0.37431 D-00 \\
0.405620-00 \\
0.437560-00 \\
0.469900-00 \\
0.50244 D 00\end{array}$ & $\begin{array}{ll}0.154710 & 02 \\
0.158280 & 02 \\
0.160860 & 02 \\
0.162400 & 02 \\
0.162860 & 02\end{array}$ & $\begin{array}{l}2.0550 \\
2.0600 \\
2.0650\end{array}$ & $\begin{array}{l}0.987820 \\
0.992970 \\
0.996090\end{array}$ & $\begin{array}{l}00 \\
00 \\
00\end{array}$ & $\begin{array}{l}0.129160 \\
0.798930 \\
0.474010-\end{array}$ & $\begin{array}{r}01 \\
00 \\
-00\end{array}$ \\
\hline
\end{tabular}

TABLE FOR THE EVALUATION OF THE FAXEN APPROXIMATION TO THE SOLUTICN OF THE LAMM EQUATION

$E T A=0.000400$

$P S I(Y, E T A)$

$0.427380-00$

$0.671490 \quad 00$

1.9350

1.9400

1.9450

1.9500

1.9550

1.9600

1.9630

1.9660

1.9680

1.9700

1.9720

1.9740

1.9760

1.9780

1.9800

1.9820

1.9840

1.9860

1.9880

1.9900

1.9920

1.9940

1.9960

1.9980

2.0000
$0.10984 \mathrm{D}-01$

$0.172520-01$

$0.263500-01$

$0.391530-01$

$0.566150-01$

$0.797030-01$

$0.966280-01$

$0.11605 \mathrm{D}-00$

$0.130460-00$

$0.146050-00$

$0.16285 \mathrm{D}-00$

$0.180850-00$

$0.200060-00$

$0.220440-00$

$0.241960-00$

$0.26458 \mathrm{D}-00$

$0.28822 \mathrm{D}-00$

$0.312820-00$

$0.338280-00$

$0.391370-00$

$0.418770-00$

$0.446570-00$

$0.474630-00$

$0.502820 \quad 00$
$0.364500-00$
0.10226001

0.15093001

0.21592001

0.29939001

0.40236001

0.52411001

0.60505001

0.69068001

0.74970001

0.80970001

$0.87014 \mathrm{D} 01$

0.93043001

C. 98994001

0.11039002

$0.115700 \quad 02$

$0.125220 \quad 02$

0.12929002

0.13282002

$0.135780 \quad 02$

0.13811002

0.13977002

$0.140760 \quad 02$

$0.141040 \quad 02$
0.10480002

0.12067002
0.53100000 0.55902000 0.58675000 $0.614050 \quad 00$

\begin{tabular}{|c|c|c|c|c|}
\hline 2.0020 & 0.531000 & 00 & 0.140610 & 02 \\
\hline 2.0040 & 0.559020 & 00 & 0.139490 & 02 \\
\hline 2.0060 & 0.586750 & 00 & 0.137690 & 02 \\
\hline 2.0080 & 0.614050 & 00 & 0.135240 & 02 \\
\hline 2.0100 & 0.640800 & 00 & 0.132160 & 02 \\
\hline 2.0120 & 0.666880 & 00 & 0.128510 & 02 \\
\hline 2.0140 & 0.692170 & 00 & 0.124340 & 02 \\
\hline 2.0160 & 0.716590 & 00 & 0.119710 & 02 \\
\hline 2.0180 & 0.740030 & 00 & 0.114670 & 02 \\
\hline 2.0200 & 0.762430 & 00 & 0.109290 & 02 \\
\hline 2.0220 & 0.783730 & 00 & 0.103650 & 02 \\
\hline 2.0240 & 0.803880 & 00 & 0.978130 & 01 \\
\hline 2.0260 & 0.822850 & 00 & 0.918410 & 01 \\
\hline 2.0280 & 0.840610 & 00 & $0.85804 \mathrm{C}$ & 01 \\
\hline 2.0300 & 0.857170 & 00 & 0.797640 & 01 \\
\hline 2.0320 & 0.872520 & 00 & 0.737800 & 01 \\
\hline 2.0340 & 0.886690 & 00 & 0.679040 & 01 \\
\hline 2.0360 & 0.899690 & 00 & 0.621840 & 01 \\
\hline 2.0380 & 0.911570 & 00 & 0.566620 & 01 \\
\hline 2.0400 & 0.922370 & 00 & 0.513730 & 01 \\
\hline 2.0450 & 0.944980 & 00 & 0.393410 & 01 \\
\hline 2.0500 & 0.962030 & 00 & 0.292000 & 01 \\
\hline 2.0550 & 0.974500 & 00 & 0.210070 & 01 \\
\hline 2.0600 & 0.983340 & 00 & 0.146470 & 01 \\
\hline 2.0650 & 0.989420 & 00 & $0.98987 \mathrm{C}$ & 00 \\
\hline $\begin{array}{l}2.0700 \\
2.0750\end{array}$ & $\begin{array}{l}0.993460 \\
0.996080\end{array}$ & $\begin{array}{l}00 \\
00\end{array}$ & $\begin{array}{l}0.648390 \\
0.41164 \mathrm{C}-\end{array}$ & $\begin{array}{r}00 \\
-00\end{array}$ \\
\hline
\end{tabular}


$E T A=0.000500$

\begin{tabular}{|c|c|c|c|c|c|c|c|c|}
\hline$Y$ & $P S I(Y, E T A)$ & DPSI $(Y, E I A$ & I/DY & $Y$ & PSI I Y, ET & (A) & DPSI $Y$, ETA & a) 10 \\
\hline $\begin{array}{l}1.9200 \\
1.9250 \\
1.9300 \\
1.9350 \\
1.9400\end{array}$ & $\begin{array}{l}0.583860-02 \\
0.904790-02 \\
0.137080-01 \\
0.203070-01 \\
0.294230-01\end{array}$ & $\begin{array}{l}0.524790 \\
0.772170 \\
0.110810 \\
0.155090 \\
0.211720\end{array}$ & $\begin{array}{l}00 \\
00 \\
01 \\
01 \\
01\end{array}$ & $\begin{array}{l}2.0020 \\
2.0040 \\
2.0060 \\
2.0080 \\
2.0100\end{array}$ & $\begin{array}{l}0.528360 \\
0.553450 \\
0.578330 \\
0.602900 \\
0.627070\end{array}$ & $\begin{array}{l}00 \\
00 \\
00 \\
00 \\
00\end{array}$ & $\begin{array}{l}0.125830 \\
0.125010 \\
0.123710 \\
0.121930 \\
0.119690\end{array}$ & $\begin{array}{l}02 \\
02 \\
02 \\
02 \\
02\end{array}$ \\
\hline $\begin{array}{l}1.9450 \\
1.9500 \\
1.9550 \\
1.9580 \\
1.9600\end{array}$ & $\begin{array}{l}0.417050-01 \\
0.57844 D-01 \\
0.785300-01 \\
0.933900-01 \\
0.104390-00\end{array}$ & $\begin{array}{l}0.281870 \\
0.366010 \\
0.463540 \\
0.527750 \\
0.572560\end{array}$ & $\begin{array}{l}01 \\
01 \\
01 \\
01 \\
01\end{array}$ & $\begin{array}{l}2.0120 \\
2.0140 \\
2.0160 \\
2.0180 \\
2.0200\end{array}$ & $\begin{array}{l}0.650750 \\
0.673860 \\
0.696320 \\
0.718060 \\
0.739020\end{array}$ & $\begin{array}{l}00 \\
00 \\
00 \\
00 \\
00\end{array}$ & $\begin{array}{l}0.117030 \\
0.113970 \\
0.110550 \\
0.106800 \\
0.102770\end{array}$ & $\begin{array}{l}02 \\
02 \\
02 \\
02 \\
02\end{array}$ \\
\hline $\begin{array}{l}1.9620 \\
1.9640 \\
1.9660 \\
1.9680 \\
1.9700\end{array}$ & $\begin{array}{l}0.116300-00 \\
0.129150-00 \\
0.142940-00 \\
0.157700-00 \\
0.173420-00\end{array}$ & $\begin{array}{l}0.618690 \\
0.665870 \\
0.713790 \\
0.762100 \\
0.810430\end{array}$ & $\begin{array}{l}01 \\
01 \\
01 \\
01 \\
01\end{array}$ & $\begin{array}{l}2.0220 \\
2.0240 \\
2.0260 \\
2.0280 \\
2.0300\end{array}$ & $\begin{array}{l}0.759150 \\
0.778400 \\
0.796740 \\
0.814150 \\
0.830600\end{array}$ & $\begin{array}{l}00 \\
00 \\
00 \\
00 \\
00\end{array}$ & $\begin{array}{l}0.984910 \\
0.940160 \\
0.893870 \\
0.846460 \\
0.798370\end{array}$ & $\begin{array}{ll}01 \\
011 \\
01 \\
011 \\
01\end{array}$ \\
\hline $\begin{array}{l}1.9720 \\
1.9740 \\
1.9760 \\
1.9780 \\
1.9800\end{array}$ & $\begin{array}{l}0.190110-00 \\
0.207760-00 \\
0.226330-00 \\
0.245800-00 \\
0.266150-00\end{array}$ & $\begin{array}{l}0.858390 \\
0.905560 \\
0.951510 \\
0.995800 \\
0.103800\end{array}$ & $\begin{array}{l}01 \\
01 \\
01 \\
01 \\
02\end{array}$ & $\begin{array}{l}2.0320 \\
2.0340 \\
2.0360 \\
2.0380 \\
2.0400\end{array}$ & $\begin{array}{l}0.84608 D \\
0.86060 D \\
0.87416 D \\
0.886760 \\
0.89844 D\end{array}$ & $\begin{array}{l}00 \\
00 \\
00 \\
00 \\
00\end{array}$ & $\begin{array}{l}0.750010 \\
0.701760 \\
0.653990 \\
0.607050 \\
0.561220\end{array}$ & $\begin{array}{ll}01 \\
01 \\
01 \\
01 \\
01 \\
01\end{array}$ \\
\hline $\begin{array}{l}1.9820 \\
1.9840 \\
1.9860 \\
1.9880 \\
1.9900\end{array}$ & $\begin{array}{l}0.287310-00 \\
0.309230-00 \\
0.331860-00 \\
0.355120-00 \\
0.378930-00\end{array}$ & $\begin{array}{l}0.107760 \\
0.111440 \\
0.114770 \\
0.117740 \\
0.120290\end{array}$ & $\begin{array}{l}02 \\
02 \\
02 \\
02 \\
02\end{array}$ & $\begin{array}{l}2.0420 \\
2.0440 \\
2.0470 \\
2.0500 \\
2.0550\end{array}$ & $\begin{array}{l}0.909220 \\
0.919130 \\
0.932420 \\
0.943960 \\
0.959690\end{array}$ & $\begin{array}{l}00 \\
00 \\
00 \\
00 \\
00\end{array}$ & $\begin{array}{l}0.516780 \\
0.473970 \\
0.413190 \\
0.356980 \\
0.274230\end{array}$ & $\begin{array}{ll}01 \\
01 \\
011 \\
01 \\
01\end{array}$ \\
\hline $\begin{array}{l}1.9920 \\
1.9940 \\
1.9960 \\
1.9980 \\
2.0000\end{array}$ & $\begin{array}{l}0.403200-00 \\
0.427860-00 \\
0.45281 D-00 \\
0.47794 D-00 \\
0.50315000\end{array}$ & $\begin{array}{l}0.122420 \\
0.124080 \\
0.125260 \\
0.125960 \\
0.126140\end{array}$ & $\begin{array}{l}02 \\
02 \\
02 \\
02 \\
02\end{array}$ & $\begin{array}{l}2.0600 \\
2.0650 \\
2.0700 \\
2.0750 \\
2.0800\end{array}$ & $\begin{array}{l}0.971620 \\
0.980460 \\
0.986840 \\
0.991330 \\
0.994420\end{array}$ & $\begin{array}{l}00 \\
00 \\
00 \\
00 \\
00\end{array}$ & $\begin{array}{l}0.205460 \\
0.150130 \\
0.107000 \\
0.743750 \\
0.504210\end{array}$ & $\begin{array}{ll}01 \\
01 \\
01 \\
01 \\
00\end{array}$ \\
\hline
\end{tabular}


$E T A=0.000600$

\begin{tabular}{|c|c|c|c|c|c|c|}
\hline Y & $\operatorname{PSI}(Y, E T A)$ & DPSI $(Y, E T A) / D Y$ & r & $P S I(Y, E T A)$ & DPSIIY,ETA & A) /DY \\
\hline $\begin{array}{l}1.9100 \\
1.9150 \\
1.9200\end{array}$ & $\begin{array}{l}0.48098 D-02 \\
0.72448 D-02 \\
0.10708 D-01\end{array}$ & $\begin{array}{l}0.403200-00 \\
0.57982000 \\
0.81661000\end{array}$ & $\begin{array}{l}2.0020 \\
2.0040 \\
2.0060 \\
2.0080 \\
2.0100\end{array}$ & $\begin{array}{ll}0.526470 & 00 \\
0.549390 & 00 \\
0.572150 & 00 \\
0.594670 & 00 \\
0.616890 & 00\end{array}$ & $\begin{array}{l}0.114900 \\
0.114270 \\
0.113270 \\
0.1111900 \\
0.1110180\end{array}$ & $\begin{array}{l}02 \\
02 \\
02 \\
02 \\
02\end{array}$ \\
\hline $\begin{array}{l}1.9250 \\
1.9300 \\
1.9350 \\
1.9400 \\
1.9450\end{array}$ & $\begin{array}{l}0.155330-01 \\
0.221150-01 \\
0.309100-01 \\
0.424210-01 \\
0.571760-01\end{array}$ & $\begin{array}{ll}0.112640 & 01 \\
0.152170 & 01 \\
0.201330 & 01 \\
0.260880 & 01 \\
0.331080 & 01\end{array}$ & $\begin{array}{l}2.0120 \\
2.0140 \\
2.0160 \\
2.0180 \\
2.0200\end{array}$ & $\begin{array}{ll}0.638720 & 00 \\
0.6601110 & 00 \\
0.681000 & 00 \\
0.701330 & 00 \\
0.721050 & 00\end{array}$ & $\begin{array}{l}0.108120 \\
0.105750 \\
0.103090 \\
0.100160 \\
0.969900\end{array}$ & $\begin{array}{l}02 \\
02 \\
02 \\
02 \\
01\end{array}$ \\
\hline $\begin{array}{l}1.9500 \\
1.9530 \\
1.9560 \\
1.9580 \\
1.9600\end{array}$ & $\begin{array}{l}0.757000-01 \\
0.888280-01 \\
0.103580-00 \\
0.114360-00 \\
0.125910-00\end{array}$ & $\begin{array}{ll}0.411510 & 01 \\
0.464200 & 01 \\
0.519720 & 01 \\
0.558050 & 01 \\
0.597210 & 01\end{array}$ & $\begin{array}{l}2.0220 \\
2.0240 \\
2.0260 \\
2.0280 \\
2.0300\end{array}$ & $\begin{array}{ll}0.740110 & 00 \\
0.758480 & 00 \\
0.776120 & 00 \\
0.793000 & 00 \\
0.809110 & 00\end{array}$ & $\begin{array}{l}0.93608 \mathrm{C} \\
0.900430 \\
0.86326 \mathrm{C} \\
0.824860 \\
0.785560\end{array}$ & $\begin{array}{l}01 \\
01 \\
01 \\
011 \\
01\end{array}$ \\
\hline $\begin{array}{l}1.9620 \\
1.9640 \\
1.9660 \\
1.9680 \\
1.9700\end{array}$ & $\begin{array}{l}0.138250-00 \\
0.151390-00 \\
0.165340-00 \\
0.180090-00 \\
0.195640-00\end{array}$ & $\begin{array}{ll}0.636990 & 01 \\
0.677170 & 01 \\
0.717480 & 01 \\
0.757660 & 01 \\
0.797430 & 01\end{array}$ & $\begin{array}{l}2.0320 \\
2.0340 \\
2.0360 \\
2.0380 \\
2.0400\end{array}$ & $\begin{array}{ll}0.824420 & 00 \\
0.838930 & 00 \\
0.852640 & 00 \\
0.865540 & 00 \\
0.877640 & 00\end{array}$ & $\begin{array}{l}0.745630 \\
0.705380 \\
0.665090 \\
0.625010 \\
0.585390\end{array}$ & $\begin{array}{ll}01 \\
011 \\
01 \\
011 \\
01\end{array}$ \\
\hline $\begin{array}{l}1.9720 \\
1.9740 \\
1.9760 \\
1.9780 \\
1.9800\end{array}$ & $\begin{array}{l}0.21198 D-00 \\
0.229090-00 \\
0.246950-00 \\
0.265530-00 \\
0.28480 D-00\end{array}$ & $\begin{array}{ll}0.836490 & 01 \\
0.874550 & 01 \\
0.911300 & 01 \\
0.946430 & 01 \\
0.979650 & 01\end{array}$ & $\begin{array}{l}2.0420 \\
2.0440 \\
2.0460 \\
2.0480 \\
2.0500\end{array}$ & $\begin{array}{ll}0.888960 & 00 \\
0.899510 & 00 \\
0.909300 & 00 \\
0.918370 & 00 \\
0.926740 & 00\end{array}$ & $\begin{array}{l}0.546450 \\
0.508410 \\
0.471450 \\
0.435710 \\
0.401350\end{array}$ & $\begin{array}{l}01 \\
01 \\
01 \\
01 \\
01\end{array}$ \\
\hline $\begin{array}{l}1.9820 \\
1.9840 \\
1.9860 \\
1.9880 \\
1.9900\end{array}$ & $\begin{array}{l}0.30471 D-00 \\
0.325210-00 \\
0.346250-00 \\
0.36779 D-00 \\
0.389740-00\end{array}$ & 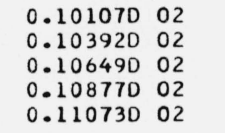 & $\begin{array}{l}2.0550 \\
2.0600 \\
2.0650 \\
2.0700 \\
2.0750\end{array}$ & $\begin{array}{ll}0.944780 & 00 \\
0.959120 & 00 \\
0.970280 & 00 \\
0.978780 & 00 \\
0.985130 & 00\end{array}$ & $\begin{array}{l}0.322100 \\
0.253170 \\
0.194890 \\
0.146930 \\
0.108490\end{array}$ & $\begin{array}{ll}0 & 1 \\
0 & 1 \\
0 & 1 \\
01 \\
01\end{array}$ \\
\hline $\begin{array}{l}1.9920 \\
1.9940 \\
1.9960 \\
1.9980 \\
2.0000\end{array}$ & $\begin{array}{l}0.412050-00 \\
0.434660-00 \\
0.457470-00 \\
0.480430-00 \\
0.50345000\end{array}$ & $\begin{array}{ll}0.1112350 & 02 \\
0.1113610 & 02 \\
0.1114500 & 02 \\
0.1115020 & 02 \\
0.1115150 & 02\end{array}$ & $\begin{array}{l}2.0800 \\
2.0850 \\
2.0900\end{array}$ & $\begin{array}{ll}0.989770 & 00 \\
0.993100 & 00 \\
0.995430 & 00\end{array}$ & $\begin{array}{l}0.784580 \\
0.555680 \\
0.385450-\end{array}$ & $\begin{array}{r}00 \\
00 \\
-00\end{array}$ \\
\hline
\end{tabular}


$E T A=0.000700$

\begin{tabular}{|c|c|c|c|}
\hline$Y$ & $P S I(Y, E T A)$ & DPSI $(Y, E T A) / D Y$ & $\mathbf{Y}$ \\
\hline $\begin{array}{l}1.9050 \\
1.9100 \\
1.9150 \\
1.9200 \\
1.9250\end{array}$ & $\begin{array}{l}0.571290-02 \\
0.829250-02 \\
0.11844 D-01 \\
0.166460-01 \\
0.23026 D-01\end{array}$ & $\begin{array}{ll}0.435040-00 \\
0.604550 & 00 \\
0.825240 & 00 \\
0.110660 & 01 \\
0.145750 & 01\end{array}$ & $\begin{array}{l}2.0020 \\
2.0040 \\
2.0060 \\
2.0080 \\
2.0100\end{array}$ \\
\hline $\begin{array}{l}1.9300 \\
1.9350 \\
1.9400 \\
1.9450 \\
1.9500\end{array}$ & $\begin{array}{l}0.31350 D-01 \\
0.42022 D-01 \\
0.55460 D-01 \\
0.720830-01 \\
0.92282 D-01\end{array}$ & $\begin{array}{ll}0.188590 & 01 \\
0.239690 & 01 \\
0.299240 & 01 \\
0.366990 & 01 \\
0.442100 & 01\end{array}$ & $\begin{array}{l}2.0120 \\
2.0140 \\
2.0160 \\
2.0180 \\
2.0200\end{array}$ \\
\hline $\begin{array}{l}1.9520 \\
1.9540 \\
1.9563 \\
1.9580 \\
1.9600\end{array}$ & $\begin{array}{l}0.101440-00 \\
0.111240-00 \\
0.121710-00 \\
0.132840-00 \\
0.144660-00\end{array}$ & $\begin{array}{ll}0.473910 & 01 \\
0.506570 & 01 \\
0.539930 & 01 \\
0.573840 & 01 \\
0.608140 & 01\end{array}$ & $\begin{array}{l}2.0220 \\
2.0240 \\
2.0260 \\
2.0280 \\
2.0300\end{array}$ \\
\hline $\begin{array}{l}1.9620 \\
1.9640 \\
1.9660 \\
1.9680 \\
1.9700\end{array}$ & $\begin{array}{l}0.157170-00 \\
0.170370-00 \\
0.18426 D-00 \\
0.198830-00 \\
0.214080-00\end{array}$ & $\begin{array}{ll}0.642660 & 01 \\
0.677200 & 01 \\
0.711560 & 01 \\
0.745530 & 01 \\
0.778890 & 01\end{array}$ & $\begin{array}{l}2.0320 \\
2.0340 \\
2.0360 \\
2.0380 \\
2.0400\end{array}$ \\
\hline $\begin{array}{l}1.9720 \\
1.9740 \\
1.9760 \\
1.9780 \\
1.9800\end{array}$ & $\begin{array}{l}0.22998 D-00 \\
0.246530-00 \\
0.26369 D-00 \\
0.28144 D-00 \\
0.29975 D-00\end{array}$ & $\begin{array}{ll}0.811420 & 01 \\
0.842900 & 01 \\
0.873110 & 01 \\
0.901820 & 01 \\
0.928810 & 01\end{array}$ & $\begin{array}{l}2.0420 \\
2.0440 \\
2.0460 \\
2.0480 \\
2.0500\end{array}$ \\
\hline $\begin{array}{l}1.9820 \\
1.9840 \\
1.9860 \\
1.9880 \\
1.9900\end{array}$ & $\begin{array}{l}0.318580-00 \\
0.337890-00 \\
0.35764 D-00 \\
0.37777 D-00 \\
0.39825 D-00\end{array}$ & $\begin{array}{ll}0.953890 & 01 \\
0.976850 & 01 \\
0.997500 & 01 \\
0.101570 & 02 \\
0.103130 & 02\end{array}$ & $\begin{array}{l}2.0520 \\
2.0550 \\
2.0600 \\
2.0650 \\
2.0700\end{array}$ \\
\hline $\begin{array}{l}1.9920 \\
1.9940 \\
1.9960 \\
1.9980 \\
2.0000\end{array}$ & $\begin{array}{l}0.419010-00 \\
0.439990-00 \\
0.461150-00 \\
0.48242 D-00 \\
0.50373000\end{array}$ & $\begin{array}{ll}0.104410 & 02 \\
0.105400 & 02 \\
0.106110 & 02 \\
0.106510 & 02 \\
0.106610 & 02\end{array}$ & $\begin{array}{l}2.0750 \\
2.0800 \\
2.0850 \\
2.0900 \\
2.0950\end{array}$ \\
\hline
\end{tabular}

PSI $(Y, E T A)$

0.52504000 $0.546270 \quad 00$ 0.56738000 0.58829000 0.60895000

0.62932000 0.64932000 0.66892000 0.68807000 0.70672000

$0.72484 \mathrm{D} 00$ $0.742390 \quad 00$ 0.75934000 $0.775660 \quad 00$ 0.79134000

0.80635000 0.82068000 0.83433000 0.84729000 $0.859550 \quad 00$

0.87113000 0.88203000 0.89226000 0.90184000 0.91078000

0.91909000 0.93045000 0.94660000 $0.959630 \quad 00$ 0.96995000

$0.977980 \quad 00$ 0.98411000 0.984110
0.98 0.99212000 $0.994580 \quad 00$
DPSI ( $Y, E T A) / D Y$

$0.106400 \quad 02$ 0.10589002 0.10509002 0.10399002 0.10261002

0.10096002 0.99054001 0.96906001 0.94534001 0.91957001

0.89196001 $0.862700 \quad 01$ $0.832020 \quad 01$ 0.80014001 0.76729001

0.73369001 0.69956001 0.66512001 0.63057001 0.59610001

0.56192001 0.52818001 0.49505001 0.46268001 0.43119001

0.40069001 0.35703001 0.29040001 0.23202001 0.18210001

0.14039001 0.10632001 0.79089000 $0.577940 \quad 00$ $0.414850-00$ 
$E T A=0.000800$

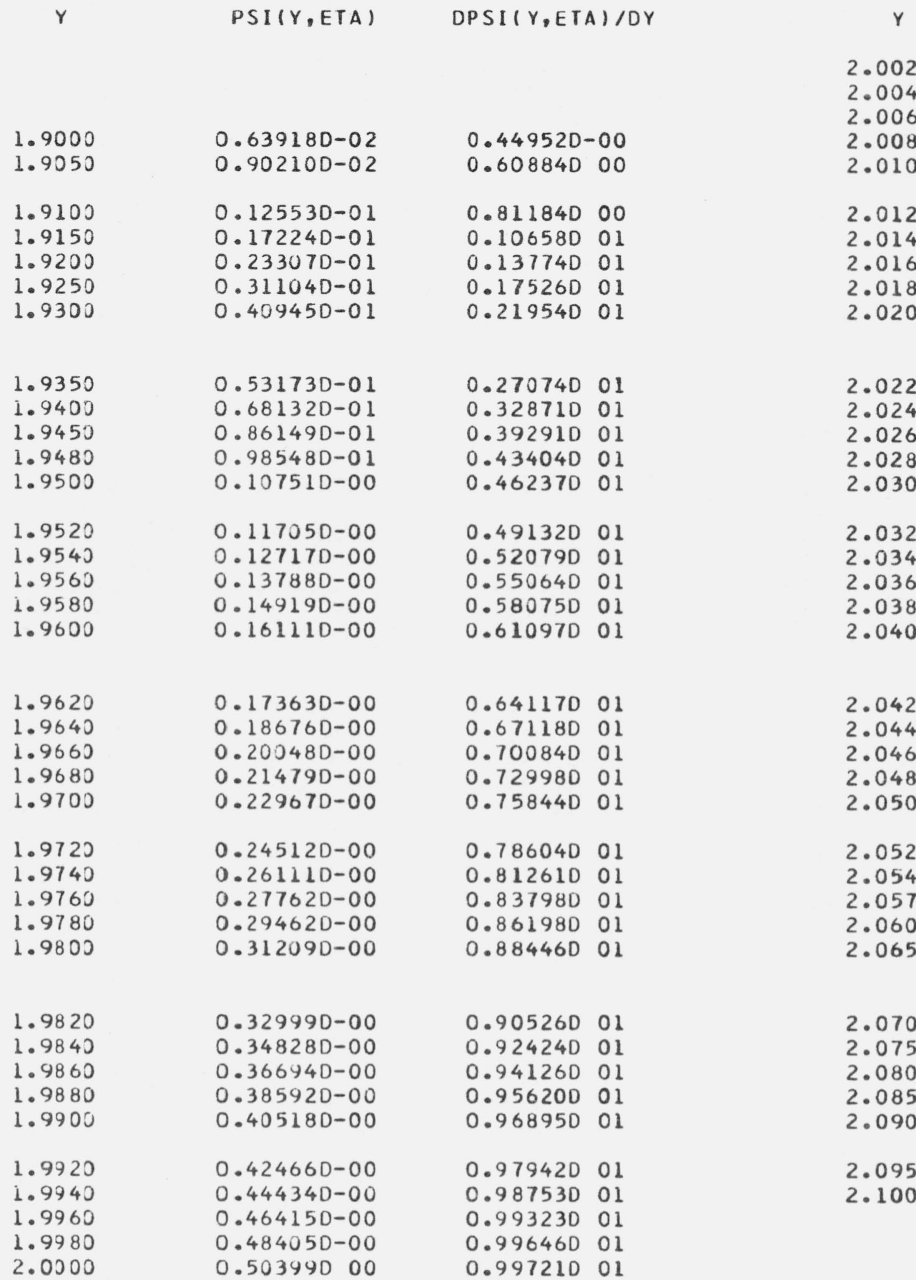

PSI (Y, ETA)

0.52392000

$0.543790 \quad 00$

0.56355000

0.58316000

0.60256000

0.62171000

0.64056000

0.65908000

0.67723000

0.69496000

$0.712240 \quad 00$ 0.72905000 0.74535000 0.76113000 0.77635000

0.79101000 0.80508000 0.81857000

0.83145000

0.84373000

$0.855400 \quad 00$ $0.866480 \quad 00$ 0.87695000 $0.88684 D 00$

0.89614000

0.90488000 0.91306000 $0.92434 D 00$ 0.93446000 0.94896000

0.96078000 0.97027000 0.97777000 0.98361000 0.98808000

0.99146000 0.99396000
DPS I ( Y, ETA ) /DY

$0.99546 \mathrm{D} 01$ 0.99124001 0.98458001 0.97551001 0.96412001

0.95048001 0.93470001 $0.91688 D 01$ 0.89716001 0.87567001

0.85256001 0.82798001 0.80211001 0.77511001 $0.747150 \quad 01$

0.71840001 0.68903001 0.65921001 0.62911001 0.59888001

0.56868001 0.53866001 0.50895001 0.47967001 0.45095001

0.42290001 0.39559001 0.35624001 0.31900001 0.26208001

0.21198001 0.16881001 0.13234001 0.10214001 0.77611000

$0.58058 D 00$ $0.427590-00$ 
$E T A=0.000900$

\begin{tabular}{|c|c|c|c|c|c|c|c|}
\hline r & $P S I(Y, E T A)$ & DPSI $(Y, E T A) / D Y$ & $\mathbf{Y}$ & PSI $(Y, E)$ & (A) & DPSIIY, ETA & A) /DY \\
\hline $\begin{array}{l}1.8900 \\
1.8950 \\
1.9000 \\
1.9050\end{array}$ & $\begin{array}{l}0.491410-02 \\
0.687020-02 \\
0.948450-02 \\
0.129300-01\end{array}$ & $\begin{array}{l}0.335560-00 \\
0.451730-00 \\
0.59974000 \\
0.78526000\end{array}$ & $\begin{array}{l}2.0020 \\
2.0040 \\
2.0060 \\
2.0080 \\
2.0100\end{array}$ & $\begin{array}{l}0.523020 \\
0.541760 \\
0.560410 \\
0.578930 \\
0.597270\end{array}$ & $\begin{array}{l}00 \\
00 \\
00 \\
00 \\
00\end{array}$ & $\begin{array}{l}0.938640 \\
0.935050 \\
0.929410 \\
0.921750 \\
0.912120\end{array}$ & $\begin{array}{ll}0 & 1 \\
0 & 1 \\
01 & 1 \\
01 & 1 \\
01\end{array}$ \\
\hline $\begin{array}{l}1.9100 \\
1.9150 \\
1.9200 \\
1.9250 \\
1.9300\end{array}$ & $\begin{array}{l}0.174090-01 \\
0.231510-01 \\
0.304110-01 \\
0.394630-01 \\
0.505940-01\end{array}$ & $\begin{array}{ll}0.101400 & 01 \\
0.129130 & 01 \\
0.162170 & 01 \\
0.200870 & 01 \\
0.245360 & 01\end{array}$ & $\begin{array}{l}2.0120 \\
2.0140 \\
2.0160 \\
2.0180 \\
2.0200\end{array}$ & $\begin{array}{l}0.615400 \\
0.633280 \\
0.650880 \\
0.668150 \\
0.685080\end{array}$ & $\begin{array}{l}00 \\
00 \\
00 \\
00 \\
00\end{array}$ & $\begin{array}{l}0.900600 \\
0.887240 \\
0.872140 \\
0.855400 \\
0.837120\end{array}$ & $\begin{array}{l}01 \\
01 \\
01 \\
01 \\
01\end{array}$ \\
\hline $\begin{array}{l}1.9350 \\
1.9400 \\
1.9450 \\
1.9480 \\
1.9500\end{array}$ & $\begin{array}{l}0.640950-01 \\
0.802420-01 \\
0.992900-01 \\
0.112200-00 \\
0.121450-00\end{array}$ & $\begin{array}{ll}0.295580 & 01 \\
0.351170 & 01 \\
0.411460 & 01 \\
0.449490 & 01 \\
0.475450 & 01\end{array}$ & $\begin{array}{l}2.0220 \\
2.0240 \\
2.0260 \\
2.0280 \\
2.0300\end{array}$ & $\begin{array}{l}0.701630 \\
0.717770 \\
0.733480 \\
0.748730 \\
0.763510\end{array}$ & $\begin{array}{l}00 \\
00 \\
00 \\
00 \\
00\end{array}$ & $\begin{array}{l}0.817400 \\
0.796390 \\
0.774190 \\
0.750940 \\
0.726770\end{array}$ & $\begin{array}{lll}0 & 1 \\
0 & 1 \\
0 & 1 \\
0 & 1 \\
0 & 1\end{array}$ \\
\hline $\begin{array}{l}1.9520 \\
1.9540 \\
1.9560 \\
1.9580 \\
1.9600\end{array}$ & $\begin{array}{l}0.131220-00 \\
0.141520-00 \\
0.152360-00 \\
0.163730-00 \\
0.175640-00\end{array}$ & $\begin{array}{ll}0.501790 & 01 \\
0.528420 & 01 \\
0.555230 & 01 \\
0.582110 & 01 \\
0.608930 & 01\end{array}$ & $\begin{array}{l}2.0320 \\
2.0340 \\
2.0360 \\
2.0380 \\
2.0400\end{array}$ & $\begin{array}{l}0.777800 \\
0.791580 \\
0.804840 \\
0.817580 \\
0.829780\end{array}$ & $\begin{array}{l}00 \\
00 \\
00 \\
00 \\
00\end{array}$ & $\begin{array}{l}0.701810 \\
0.676210 \\
0.650100 \\
0.623610 \\
0.596870\end{array}$ & $\begin{array}{ll}0 & 1 \\
0 & 1 \\
0 & 1 \\
0 & 1 \\
01 & 1\end{array}$ \\
\hline $\begin{array}{l}1.9620 \\
1.9640 \\
1.9660 \\
1.9680 \\
1.9700\end{array}$ & $\begin{array}{l}0.188090-00 \\
0.201060-00 \\
0.214560-00 \\
0.228570-00 \\
0.243080-00\end{array}$ & $\begin{array}{ll}0.635570 & 01 \\
0.661910 & 01 \\
0.687810 & 01 \\
0.713130 & 01 \\
0.737750 & 01\end{array}$ & $\begin{array}{l}2.0420 \\
2.0440 \\
2.0460 \\
2.0480 \\
2.0500\end{array}$ & $\begin{array}{l}0.841450 \\
0.852580 \\
0.863180 \\
0.873240 \\
0.882780\end{array}$ & $\begin{array}{l}00 \\
00 \\
00 \\
00 \\
00\end{array}$ & $\begin{array}{l}0.570010 \\
0.543150 \\
0.516410 \\
0.489900 \\
0.463710\end{array}$ & $\begin{array}{ll}01 \\
01 \\
01 \\
01 \\
01\end{array}$ \\
\hline $\begin{array}{l}1.9720 \\
1.9740 \\
1.9760 \\
1.9780 \\
1.9800\end{array}$ & $\begin{array}{l}0.258080-00 \\
0.273540-00 \\
0.289440-00 \\
0.305770-00 \\
0.322490-00\end{array}$ & $\begin{array}{ll}0.761520 & 01 \\
0.784310 & 01 \\
0.806000 & 01 \\
0.826440 & 01 \\
0.845530 & 01\end{array}$ & $\begin{array}{l}2.0520 \\
2.0540 \\
2.0570 \\
2.0600 \\
2.0650\end{array}$ & $\begin{array}{l}0.891790 \\
0.900300 \\
0.912130 \\
0.922870 \\
0.938520\end{array}$ & $\begin{array}{l}00 \\
00 \\
00 \\
00 \\
00\end{array}$ & $\begin{array}{l}0.437950 \\
0.412700 \\
0.375970 \\
0.340790 \\
0.286130\end{array}$ & $\begin{array}{ll}0 & 1 \\
0 & 1 \\
0 & 1 \\
0 & 1 \\
01 & 1\end{array}$ \\
\hline $\begin{array}{l}1.9820 \\
1.9840 \\
1.9860 \\
1.9880 \\
1.9900\end{array}$ & $\begin{array}{l}0.339580-00 \\
0.357010-00 \\
0.374730-00 \\
0.392730-00 \\
0.41096 D-00\end{array}$ & $\begin{array}{ll}0.863130 & 01 \\
0.879150 & 01 \\
0.893470 & 01 \\
0.906010 & 01 \\
0.916700 & 01\end{array}$ & $\begin{array}{l}2.0700 \\
2.0750 \\
2.0800 \\
2.0850 \\
2.0900\end{array}$ & $\begin{array}{l}0.951580 \\
0.962310 \\
0.971020 \\
0.977990 \\
0.983480\end{array}$ & $\begin{array}{l}00 \\
00 \\
00 \\
00 \\
00\end{array}$ & $\begin{array}{l}0.236920 \\
0.193470 \\
0.155810 \\
0.123750 \\
0.969360\end{array}$ & $\begin{array}{ll}01 \\
01 \\
01 \\
01 \\
00\end{array}$ \\
\hline $\begin{array}{l}1.9920 \\
1.9940 \\
1.9960 \\
1.9980 \\
2.0000\end{array}$ & $\begin{array}{l}0.429390-00 \\
0.44797 D-00 \\
0.46666 D-00 \\
0.485430-00 \\
0.50423000\end{array}$ & $\begin{array}{ll}0.925440 & 01 \\
0.932200 & 01 \\
0.936930 & 01 \\
0.939580 & 01 \\
0.940160 & 01\end{array}$ & $\begin{array}{l}2.0950 \\
2.1000 \\
2.1050 \\
2.1100\end{array}$ & $\begin{array}{l}0.987760 \\
0.991040 \\
0.993530 \\
0.995380\end{array}$ & $\begin{array}{l}00 \\
00 \\
00 \\
00\end{array}$ & $\begin{array}{l}0.748820 \\
0.570470 \\
0.428610- \\
0.317590-\end{array}$ & $\begin{array}{r}00 \\
00 \\
-00 \\
-00\end{array}$ \\
\hline
\end{tabular}


$E T A=0.001000$

\begin{tabular}{|c|c|c|c|c|c|c|c|}
\hline Y & $P S I(Y, E T A)$ & DPSI $(Y, E T A) / D Y$ & $Y$ & PSIIY,EI & (A) & DPSIIY,ETA & A) $/ 0$ \\
\hline $\begin{array}{l}1.8850 \\
1.8900 \\
1.8950 \\
1.9000 \\
1.9050\end{array}$ & $\begin{array}{l}0.52342 \mathrm{D}-02 \\
0.717910-02 \\
0.97356 \mathrm{D}-02 \\
0.13054 \mathrm{D}-01 \\
0.173090-01\end{array}$ & $\begin{array}{l}0.336730-00 \\
0.445500-00 \\
0.58210000 \\
0.75112000 \\
0.95719000\end{array}$ & $\begin{array}{l}2.0020 \\
2.0040 \\
2.0060 \\
2.0080 \\
2.0100\end{array}$ & $\begin{array}{l}0.522290 \\
0.540070 \\
0.557770 \\
0.575360 \\
0.592800\end{array}$ & $\begin{array}{l}00 \\
00 \\
00 \\
00 \\
00\end{array}$ & $\begin{array}{l}0.890560 \\
0.887450 \\
0.882580 \\
0.875990 \\
0.867710\end{array}$ & $\begin{array}{ll}01 \\
01 \\
01 \\
01 \\
01 \\
01\end{array}$ \\
\hline $\begin{array}{l}1.9100 \\
1.9150 \\
1.9200 \\
1.9250 \\
1.9300\end{array}$ & $\begin{array}{l}0.226950-01 \\
0.294300-01 \\
0.377480-01 \\
0.478910-01 \\
0.601080-01\end{array}$ & $\begin{array}{ll}0.120470 & 01 \\
0.149730 & 01 \\
0.183780 & 01 \\
0.222780 & 01 \\
0.266710 & 01\end{array}$ & $\begin{array}{l}2.0120 \\
2.0140 \\
2.0160 \\
2.0180 \\
2.0200\end{array}$ & $\begin{array}{l}0.610060 \\
0.627100 \\
0.643900 \\
0.660430 \\
0.676650\end{array}$ & $\begin{array}{l}00 \\
00 \\
00 \\
00 \\
00\end{array}$ & $\begin{array}{l}0.857790 \\
0.846290 \\
0.833280 \\
0.818820 \\
0.803020\end{array}$ & $\begin{array}{ll}0 & 1 \\
0 & 1 \\
01 \\
01 \\
01 \\
01\end{array}$ \\
\hline $\begin{array}{l}1.9350 \\
1.9400 \\
1.9450 \\
1.9480 \\
1.9500\end{array}$ & $\begin{array}{l}0.74640 D-01 \\
0.917120-01 \\
0.111520-00 \\
0.124780-00 \\
0.134210-00\end{array}$ & $\begin{array}{ll}0.315330 & 01 \\
0.368180 & 01 \\
0.424550 & 01 \\
0.459670 & 01 \\
0.483480 & 01\end{array}$ & $\begin{array}{l}2.0220 \\
2.0240 \\
2.0260 \\
2.0280 \\
2.0300\end{array}$ & $\begin{array}{l}0.692540 \\
0.708080 \\
0.723240 \\
0.738000 \\
0.752350\end{array}$ & $\begin{array}{l}00 \\
00 \\
00 \\
00 \\
00\end{array}$ & $\begin{array}{l}0.785940 \\
0.767690 \\
0.748370 \\
0.728070 \\
0.706910\end{array}$ & $\begin{array}{ll}0 & 1 \\
0 & 1 \\
01 \\
01 \\
01 \\
01\end{array}$ \\
\hline $\begin{array}{l}1.9520 \\
1.9540 \\
1.9560 \\
1.9580 \\
1.9600\end{array}$ & $\begin{array}{l}0.14412 D-00 \\
0.154510-00 \\
0.165390-00 \\
0.176740-00 \\
0.188580-00\end{array}$ & $\begin{array}{ll}0.507500 & 01 \\
0.531650 & 01 \\
0.555830 & 01 \\
0.579960 & 01 \\
0.603920 & 01\end{array}$ & $\begin{array}{l}2.0320 \\
2.0340 \\
2.0360 \\
2.0380 \\
2.0400\end{array}$ & $\begin{array}{l}0.766280 \\
0.779750 \\
0.792770 \\
0.805320 \\
0.817400\end{array}$ & $\begin{array}{l}00 \\
00 \\
00 \\
00 \\
00\end{array}$ & $\begin{array}{l}0.685000 \\
0.662440 \\
0.639340 \\
0.615810 \\
0.591970\end{array}$ & $\begin{array}{ll}0 & 1 \\
0 & 1 \\
0 & 1 \\
0 & 1 \\
0 & 1\end{array}$ \\
\hline $\begin{array}{l}1.9620 \\
1.9640 \\
1.9660 \\
1.9680 \\
1.9700\end{array}$ & $\begin{array}{l}0.20090 D-00 \\
0.213680-00 \\
0.226930-00 \\
0.240630-00 \\
0.254770-00\end{array}$ & $\begin{array}{ll}0.627620 & 01 \\
0.650950 & 01 \\
0.673790 & 01 \\
0.696040 & 01 \\
0.717590 & 01\end{array}$ & $\begin{array}{l}2.0420 \\
2.0440 \\
2.0460 \\
2.0480 \\
2.0500\end{array}$ & $\begin{array}{l}0.829000 \\
0.840110 \\
0.850750 \\
0.860900 \\
0.870570\end{array}$ & $\begin{array}{l}00 \\
00 \\
00 \\
00 \\
00\end{array}$ & $\begin{array}{l}0.567910 \\
0.543740 \\
0.519560 \\
0.495460 \\
0.471540\end{array}$ & $\begin{array}{ll}0 & 1 \\
0 & 1 \\
0 & 1 \\
01 & 1 \\
01\end{array}$ \\
\hline $\begin{array}{l}1.9720 \\
1.9740 \\
1.9760 \\
1.9780 \\
1.9800\end{array}$ & $\begin{array}{l}0.269330-00 \\
0.284300-00 \\
0.299650-00 \\
0.31537 D-00 \\
0.331430-00\end{array}$ & $\begin{array}{ll}0.738330 & 01 \\
0.758160 & 01 \\
0.776950 & 01 \\
0.794630 & 01 \\
0.811080 & 01\end{array}$ & $\begin{array}{l}2.0520 \\
2.0540 \\
2.0570 \\
2.0600 \\
2.0650\end{array}$ & $\begin{array}{l}0.879760 \\
0.888480 \\
0.900710 \\
0.911920 \\
0.928460\end{array}$ & $\begin{array}{l}00 \\
00 \\
00 \\
00 \\
00\end{array}$ & $\begin{array}{l}0.447880 \\
0.424550 \\
0.390350 \\
0.357300 \\
0.305240\end{array}$ & $\begin{array}{ll}0 & 1 \\
0 & 1 \\
0 & 1 \\
0 & 1 \\
0 & 1\end{array}$ \\
\hline $\begin{array}{l}1.9820 \\
1.9840 \\
1.9860 \\
1.9880 \\
1.9900\end{array}$ & $\begin{array}{l}0.34780 D-00 \\
0.364470-00 \\
0.381390-00 \\
0.398550-00 \\
0.41590 D-00\end{array}$ & $\begin{array}{ll}0.826220 & 01 \\
0.839970 & 01 \\
0.852230 & 01 \\
0.862950 & 01 \\
0.872060 & 01\end{array}$ & $\begin{array}{l}2.0700 \\
2.0750 \\
2.0800 \\
2.0850 \\
2.0900\end{array}$ & $\begin{array}{l}0.942510 \\
0.954300 \\
0.964060 \\
0.972040 \\
0.978480\end{array}$ & $\begin{array}{l}00 \\
00 \\
00 \\
00 \\
00\end{array}$ & $\begin{array}{l}0.257530 \\
0.214580 \\
0.176570 \\
0.143490 \\
0.115160\end{array}$ & $\begin{array}{lll}0 & 1 \\
0 & 1 \\
0 & 1 \\
0 & 1 \\
0 & 1\end{array}$ \\
\hline $\begin{array}{l}1.9920 \\
1.9940 \\
1.9960 \\
1.9980 \\
2.0000\end{array}$ & $\begin{array}{l}0.43342 D-00 \\
0.451070-00 \\
0.46881 D-00 \\
0.48662 D-00 \\
0.50446000\end{array}$ & $\begin{array}{ll}0.879500 & 01 \\
0.885230 & 01 \\
0.889220 & 01 \\
0.891450 & 01 \\
0.891890 & 01\end{array}$ & $\begin{array}{l}2.0950 \\
2.1000 \\
2.1050 \\
2.1100 \\
2.1150\end{array}$ & $\begin{array}{l}0.983630 \\
0.987680 \\
0.990830 \\
0.993250 \\
0.995090\end{array}$ & $\begin{array}{l}00 \\
00 \\
00 \\
00 \\
00\end{array}$ & $\begin{array}{l}0.912770 \\
0.714470 \\
0.552310 \\
0.421650- \\
0.317900-\end{array}$ & $\begin{array}{r}00 \\
00 \\
00 \\
-00 \\
-00\end{array}$ \\
\hline
\end{tabular}

Thanks are due to Dr. Robert Jastrow, Director, Goddard Institute for Space Studies, for use of the Institute's computing facilities.

\section{References}

[1] H. Fujita, Mathematical Theory of Sedimentation Analysis, (Academic Press, New York, 1962).

[2] I. H. Billick, G. H. Weiss, The solution to a nonlinear Lamm equation in the Faxén approximation, to be published.

[3] A. Opler, N. K. Hiester, Tables for Predicting the Performance of Fixed Bed Ion Exchange and Similar Mass Transfer Processes, Stanford Research Institute (1954).

[4] S. Goldstein, On the mathematics of exchange processes in fixed columns. I. Mathematical solutions and asymptotic expansions, Proc. Roy. Soc. A219, 151 (1953).

[5] S. O. Rice, Mathematical analysis of random noise, Bell System Tech. J. 24, 46 (1945).

[6] H. C. Thomas, Heterogeneous ion exchange in a flowing system, J. Am. Chem. Soc. 66, 1664 (1944).

(Paper 70B1-171) 


\section{Publications of the National Bureau of Standards*}

\section{Selected Abstracts}

A new differential operator of the pure vave type, J. E. Lagnese, J. Different. Eq. 1, No. 2, 171-187 (Apr. 1965).

Consider the differential operator $\mathscr{L} u=u+c(t) u\left(=\partial_{t}^{2}-\sum_{i=1}^{7} \partial_{x_{i}}^{2}\right)$ with $c(t)$ analytic on some open, connected interval of the real $t$-axis. Under this requirement, we seek to determine explicitly the form of $c(t)$ for which $\mathscr{L}$ satisfies Huygen's principle in the sense of "Hadamard's premise." (Mathisson called such operators "pure wave".) Theorem. $\mathscr{L}$ is of pure waves if, and only if, it is equivalent to one of the following operators: $\mathrm{u}, \mathrm{u}-\frac{2}{\mathrm{t}^{2}} \mathrm{u}, \mathrm{u}-\frac{6}{\mathrm{t}^{2}} \mathrm{u}, \mathrm{u}-\frac{6 \mathrm{t}\left(2+\mathrm{t}^{3}\right)}{\left(1-\mathrm{t}^{3}\right)^{2}} \mathrm{u}$.

This theorem extends earlier results along this line and presents a new, and substantially more complicated, counterexample to "Hadamard's conjecture," namely the operator $u-6 t\left(2+t^{3}\right) /(1$ $\left.-t^{3}\right)^{2} u$.

Aggregation in matrix models of resource flows, D. Rosenblatt, Am. Stat. 19, No. 3, 36-39 (June 1965).

The aggregation of finite substochastic systems of the form $x$ $(I-A)=w, A$ a square and substochastic matrix and $w$ a nonnegative vector, is considered from normative and descriptive standpoints in connection with models of resource flows. Certain of the fundamental functional equations of the theory of aggregation are derived and are given a graph theoretic interpretation.

An application of the inverse $Z$-transform theory to the synthesis of linear antenna arrays, M. T. Ma, IEEE Trans. Ant. Prop. AP-12, No. 6, 798-799 (Nov. 1964).

It has been known that the $Z$-transform theory can be applied as a new technique for analyzing linear array problems. The reverse problem, synthesizing linear arrays by applying the inverse $Z$ transform theory, has not yet been reported in the literature.

This communication demonstrates that the job of synthesizing an antenna array, when certain conditions are satisfied by the given radiation pattern, can be accomplished particularly as a result of applying the inverse $Z$-transform theory originally developed for sampled-data systems, and that the error occurring between the synthesized and specified patterns can be estimated.

Spacetime coordinate systems, G. E. Hudson, Proc. Intern. Conf. Chronometry, pp. 197-221 (Lausanne, Switzerland, June 1964).

The principle of covariance is sometimes said to preclude the necessity for specifying a set of coordinates operationally. A simple example illustrates that this is not the case. This leads to the basic problem of specifying coordinate systems of physical use and interest over the vast reaches of spacetime. Examples of two operational definitions for spacetime networks associated with the surface of a spinning sphere serve to make definite some of the requirements, and show the importance and use of the general Doppler effect. For space navigational purposes, the gravitational fields, medium refraction and dispersion, and instrument uncertainty are discussed.

The point of view we have adopted here stems from, but is not limited to, a consideration of general relativistic effects and uses much of the formalism of relativity theory. It suggests moreover, a new general approach to time synchronization, space communication, and tracking problems, which is reminiscent of the concept of "homologue" space introduced by various authors to eliminate major refraction effects in a medium, or of Fock's concept of harmonic coordinates. Of more importance is the demonstration that a generalized range and range-rate coordinate system introduced here can be identified with a class of "null" coordinate systems previously mentioned by Synge.
Correlated walk and diffusion equations in a driving force, J. R. Manning, Phys. Rev. 139, No. 1A, Al26-A135 (July 5, 1965). The correlation factor for diffusion by a vacancy mechanism arises because an atom can exchange with a given vacancy more than once. This results in a series of correlated exchanges between the atom and vacancy. In order to include correlation effects in the diffusion equations, one must allow the atom sufficient time to complete any corrrlated series of jumps. With this in mind, the basic diffusion equations are derived in terms of the number $N$ of correlated series. A complete description of planar diffusion then can be presented in terms of effective jump frequencies, where each jump is independent of previous jumps. The resulting equations are correct to first order in small quantities. This is shown to be true even where there are driving forces and a diffusion coefficient gradient. Final expressions for the diffusion coefficient, correlation factor, drift velocity, and diffusion flux are valid for diffusion in any direction in cubic crystals and along particular directions in noncubic crystals. As an example, effects from a chemical concentration gradient are discussed.

Generalized master equation for quantum mechanical systems to all orders in the density, J. Weinstock, Phys. Rev. 136, $2 d$ series, No. 4A, A879-A888 (Nov. 1964).

An exact generalized master equation is derived for a large quantum mechanical system in the form of a power series in the density. This derivation is a quantum mechanical generalization of a previous work by the author for classical systems. The quantum equation can be viewed as a time-dependent analog of the virial expansion of the quantum mechanical partition function for both degenerate systems (B.E. or F.D. statistics) and non-degenerate systems. The coefficients of the series, in the quantum equation, are explicitly given in terms of operators (Green functions) which are determined by the dynamics of isolated groups of particles and are convergent functions of the interaction potential. Equations are obtained for the off-diagonal elements of the density matrix as well as for the diagonal elements. The equation for the diagonal elements is shown to reduce to a Markoffian master equation for asymptotically long times, and an explicit expression is obtained for the "scattering" operator of this asymptotic equation.

Master equations and Markov processes, I. Oppenheim and K. E. Shuler, Phys. Rev. 138, No. 4B, B117-B1011 (May 24, 1965). The processes described by generalized master equations (GME), derived from the Liouville equation on the basis of various physical and dynamic arguments, have been termed Markovian or nonMarkovian depending upon whether the GME did not or did involve an explicit time integration. We have shown here that these designations are not in accord with the (very specific) mathematical definition of a Markov and a non-Markovian process. We suggest that the much more appropriate terms Pauli process (or equation) and non-Paulian process (or equation) be used instead to designate processes described by the GME.

Analogies between theories of antenna arrays and passive networks, M. T. Ma., IEEE Intern. Conv. Record 13, Pt. 5, 150154 (Mar. 1965).

This paper demonstrates the analogy between two different fields, antenna arrays and passive networks, which are generally not known to each other. Successful application, either directly or with modification, of various known techniques for synthesizing a passive network, when an impulse response is specified, to the synthesis of linear antenna arrays with amplitude excitations, phase distributions and element spacings as the controlling parameters, when a desired radiation pattern is given, is presented with many interesting examples. 
Approximations to the distribution of quadratic forms, M. M. Siddiqui, Ann. Math. Stat. 36, No. 2, 677-682 (Apr. 1965). Let $Q=1 / 2 \sum_{j=1}^{2 k} a_{j} X_{j}^{2}$, where $0<a_{1} \leqslant a_{2} \leqslant \ldots \leqslant a_{2 k}$ are constants and $X_{1}, \ldots, X_{2 k}$ are independent $N(0,1)$ variates. If $a_{j}$ 's are equal within groups of even sizes the distribution of $Q$ can be evaluated exactly: otherwise some approximations to this distribution are needed. In the present paper an approximation to $F(x)=\operatorname{Pr}(Q>x)$ is obtained by bounding $Q$ by $Q_{1}$ and $Q_{2}$, where $Q_{1}$ and $Q_{2}$ are quadratic forms whose exact distributions can be evaluated. Let $F_{i}(x)$ $=\operatorname{Pr}\left(Q_{i}>x\right), \quad i=1,2$, and let $\hat{F}(x)=(1-0) \quad F_{i}(x)+0 F_{2}(x)$. The approximation to $F(x)$ is obtained by minimizing $d(F, F)$ for variations of 0 where $d(\ldots)$ is the distance function of the metric space $L^{2}$ $(0, \infty)$.

Estimation for a one-parameter exponential model, J. A. Speckman and R. G. Cornell, J. Amer. Stat. Assoc. 60, 560-572 (1965).

The partial totals estimation procedure is presented, illustrated and evaluated for the model $y=\exp (-\rho t)+e$ when the values of $t$ are equally spaced. Tables of estimates using this estimation procedure are given for the case where the smallest value of $t$ is zero. The evaluation consists of an analytical investigation of the large sample properties of the partial totals estimator and a comparative study of its small sample properties relative to three other estimators based on Monte Carlo results where it is assumed either that $y$ is a binomial proportion or that $y$ is a proportion with variance proportional to a binomial variance.

Inequalities for permanents and permanental minors, R. A. Brualdi and M. Newman, Proc. Cambridge Phil. Soc. 61, pt. 3, 741-746 (Jan. 26, 1965).

The principal result of this paper is that for all non-negative doublystochastic matrices $A$ and all $\alpha$ such that $0 \leqslant \alpha \leqslant 1, \operatorname{per}(\alpha I+(1-\alpha) A)$ $\leqslant \alpha+(1-\alpha) \operatorname{per}(A)$, where per $(A)$ denotes the permanent of $A$.

On the binary collision expansion of the classical $\mathbf{N}$-body Green's function, J. Weinstock, Phys. Rev. 126, No. 1, 341-344 (Apr. 1, 1962).

The explicit time integrations in the formal Binary Collision Expansion of the classical $N$-body Green's function are performed to obtain a product of binary collision operators which bears a strong resemblance to the Mayer product of $f_{i j}$ 's. These integrations are exact for hard sphere interactions and are expected to be a good approximation for finite but short range pair interactions. The resulting expansion can be averaged over configuration space to obtain a transport equation for a dense gas in analogy with the cluster expansion of the classical partition function.

On matching problems, J. Edmonds, A. J. Goldman, C. Witzgall, C. T. Zahn, Jr., Proc. ARO Working Group on Computer, ARU-D Report 65-1, pp. 46-50 (Office Cinlef Res. and Develop., Feb. 1964).

Combinatorial optimization problems, although finite, remain intracable in practice so long as all known solution methods involve an amount of labor which increases exponentially with problem size. The present paper reports the recent development, by members of the Combinatorial Mathematics Project at the National Bureau of Standards, of efficient solution and checking algorithms for one class of problems. The prototype of this class is the problem of finding in a linear graph a set of pairwise disjoint edges (a "matching") with as many edges as possible; several generalizations are also discussed.

Paths. trees, and flowers, J. Edmonds, Can. J. Math. 17, 449467 (1965).

A matching in a graph is a subset of its edges such that no two meet the same vertex. The theory of matchings is treated. In particular, an efficient algorithm is described for finding in a given graph a matching of maximum cardinality. The Konig theorem is generalized to arbitrary (non-bipartite) graphs.

Realization of semi-multipliers as multipliers, H. Fell and A. J. Goldman, Am. Math. Mo. 72, No. 6, 641 (June-July 1965).

The semi-multipliers of a linear associative algebra $A$ are linear transformations obeying a generalization of the formula imposed on the multipliers of $A$ by the associative law. Buck showed that $A$ could be imbedded as an ideal in a very large algebra $B$ so that each semi-multiplier of $A$ was realized as a multiplier in $B$, and asked how to construct a smaller algebra $B$ with the same property. This note presents such a construction.

\section{Some remarks on certain generalized Dedekind sums,}

H. Rademacher, Acta Arithmetica 9, Sec. 1, 97-105 (1964).

Some years ago the German mathematician C. Meyer introduced a certain generalization of the Dedekind sums. This paper has as its aim to formulate the "true" generalization, which lies behind all the previous attempts. In this new formulation the theorems become not only general but also really transparent. A reciprocity theorem is to be expected in this theory and is indeed fully established. The paper closes with a hint at a useful Euclidean algorithm.

The paper is dedicated to L. J. Mordell (who himself has made contributions to the theory of Dedekind sums). It is an invited paper for an anniversary volume.

Stochastic theory of diffusion in a plasma across a magnetic field, C. M. Tchen, Proc. Intern. Symp. Diffusion, Feldafing, Germany, June 29-July 3, 1964, pp. 118-123 (1964).

A stochastic theory is proposed to investigate the diffusion across a constant magnetic field for the cases of collision and collective oscillations. A general formula is obtained, which shows the two cases separately, and the underlying assumptions permitting such a separation are clarified. It is found that the diffusion by collective oscillations is determined by the wave energy, while the collisional diffusion is determined by the thermal energy.

Electromagnetic properties of a quantized relativistic electron-positron gas, L. A. Steinert, Il Nuovo Cimento, Serie X 36, 935-95.3 (Apr. 1965).

A relativistic quantum statistical description is given of electromagnetic wave propagation in a uniformly magnetized electronpositron gas, with specific attention devoted to long wavelength propagation as determined by the "collective" approximation. Collisionless absorption effects derived from relativity theory are compared with those obtained from the corresponding nonrelati vistic treatment, showing that the two theories predict substantially different effects even for "nonrelativistic" temperatures. Contrasts appear in the predicted characteristics of the cyclotron resonance absorption effect, including the fact that Landau damping is completely nonexistent in the relativistic gas for waves propagating at phase velocities exceeding the velocity of light.

Wave functions for anharmonic oscillators by perturbation methods, A. M. Shorb, R. Schroeder, and E. R. Lippincott, J. Chem. Phys. 37, 1043 (Sept. 1, 1962).

Approximate wave functions and the corresponding energies are given in tabular form for anharmonic potentials which can be expanded in polynomial series up to the sixth power in the displacement, $x$.

Localized-induction concept on a curved vortex and motion of an elliptic vortex ring, R. J. Arms and F. R. Hama, Phys. Fluids 8, No. 4, 553-559 (Apr. 1965).

The localized-induction concept for the induction effect of a smooth curved vortex on itself is derived. This concept is applicable to the limiting case of a vortex filament of infinitesimal core size and of negligible long-distance effect, and was already successfully utilized in the investigations of the motion and deformation of a curved vortex filament given various initial configurations. Two theorems obtained under this concept are that the arc length of a vortex filament and the projected area of a closed vortex filament are both invariant with respect to time. These theoretical predictions are examined by a numerical analysis of the motion of an initially plane elliptic vortex ring of various eccentricities.

Random-walk model of chain-polymer adsorption at a surface, R. J. Rubin, J. Chem. Phys. 43, No. 7, 2392-2407 (Oct. 1, 1965). A random walk lattice model of adsorption of an isolated polymer chain at a solution surface is investigated. On neglecting the self-excluded volume, a number of one-dimensional characteristics of the monomer unit distribution are determined analytically in the limit of long polymer chains. On assuming that one end of the poly. 
mer chain lies in the surface layer, the mean number of monomer units adsorbed in the surface layer $\nu(\theta, N)$ and the mean distance of the other end of the chain from the surface layer $z(\theta, N)$ are determined, where $N$ is the number of monomer units in the chain and $\theta$ is the adsorption energy of each monomer unit in the surface layer measured in units of $k T$. The lattice models considered include the simple cubic, hexagonal, close-packed, face-centered cubic, and body-centered cubic lattices. In the limit in which $N \rightarrow \infty$, both $\nu(\theta, N)$ and $z(\theta, N)$ exhibit a very interesting discontinuity at a latticedependent adsorption energy $\theta_{c}$. For example for $\theta>\theta_{c}, \nu(\theta, N)$ (which is also proportional to the average adsorption energy of a polymer chain) is proportional to $N$. For $\theta<\theta_{c}, \nu(\theta, N)$ is proportional to a constant of order unity; and for $\theta=\theta_{c}, \nu(\theta, N)$ is proportional to $N^{1 / 2}$. It is shown that the probability distribution of the end of the chain decreases exponentially with increasing distance from the surface layer for $\theta>\boldsymbol{\theta}_{c}$.

In addition, the mean number of monomer units in the $k$ th layer from the surface is determined for $N>>1$ and $\theta>\theta_{c}$ and is found to decrease exponentially with increasing $k$. In effect, for $\theta>\theta_{c}$ the polymer chain exists in an adsorbed state. An improvement in the model, which includes short range correlation between successive steps in the random walk description, is also considered.

Interaction between configurations with several open shells, U. Fano, Phys. Rev. 140, No. 1A, A67-A75 (Oct. 4, 1965).

Antisymmetrized product wave functions are written for atomic configurations with an arbitrary number of partially or entirely filled shells. Interaction matrix elements between such configurations are calculated and a numerical evaluation is given for the autoionization of an inner excitation level of neon. A suitable notation keeps the analytical complexities within reasonable bounds.

\section{Other NBS Publications}

\section{J. Res. NBS 69A (Phys. and Chem.), No. 6 (Nov.-Dec. 1965),} 75 cents.

Arc measurement of some argon transition probabilities. C. H. Popenoe and J. B. Shumaker, Jr.

Theoretical interpretation of the third spectrum of gold (Au III). Y. Shadmi.

Photopolarographic behavior of inorganic depolarizers. R. A. Durst and J. K. Taylor.

Spectral structure of critical opalescence: binary mixture. R. D. Mountain.

Irregularities in the NBS (1955) provisional temperature scale. H. M. Roder.

Isotherms determined by the National Bureau of Standards acoustical thermometer in the liquid helium temperature range. G. Cataland and H. Plumb.

Synthesis of D-glucose-3.14 $C$ and related compounds. H. L. Frush, L. T. Sniegoski, N. B. Holt, and H. S. Isbell.

Correlation of large longitudinal deformations with different histories. L. J. Zapas and T. Craft.

Crystallography of tetracalcium phosphate. W. E. Brown and E. F. Epstein.

Electrode potentials in fused systems XII. Measurement of cation concentration in molten salts using glass membrane electrodes.

K. H. Stern and S. E. Meador.

Radio Sci. J. Res. NBS/USNC-URSI 69D, No. 12 (December 1965), \$1.00

Symposium on Planetary Atmospheres and Surfaces, May 1965:

I Session: Jupiter, as observed at long radio waves.

II Session: Jupiter, as observed at short radio waves.

III Session: Passive radio observations of Venus, Saturn, Mercury, Mars, and Uranus.

IV Session: Passive radio observations of the Moon.

V Session: Radar observations of the planets

VI Session: Radar observations of the Moon.

Oxidation of polycyclic, aromatic hydrocarbons. A review of the literature, R. S. Tipson, NBS Mono. 87 (Sept. 17, 1965), 40 cents. Spot diagrams for the prediction of lens performance from design data, O. N. Stavroudis and L. E. Sutton; NBS Mono. 93 (Sept. 7, 1965), 75 cents.

Thermodynamic and related properties of parahydrogen from the triple point to $100{ }^{\circ} \mathrm{K}$ at pressures to 340 atmospheres, H. M. Roder, L. A. Weber, and R. D. Goodwin, NBS Mono. 94 (Aug. $10,1965), 75$ cents.

Specifications, tolerances, and other technical requirements for commercial weighing and measuring devices, NBS Handb. 44-3d edition (Oct. 12, 1965), \$2.00. Supersedes NBS Handb. 44-2d edition.

Standard Reference Materials: Catalog and price list of standard materials issued by the National Bureau of Standards, NBS Misc. Publ. 260, (Oct. 1, 1965), 45 cents. Supersedes NBS Misc. Publ. 241.

Standard Reference Materials: Methods for the chemical analysis of white cast iron standards, J. I. Shultz, NBS Misc. Publ. 260-6 (July 16, 1965), 45 cents.

Hydraulic research in the United States 1965, ed. H. K. Middleton, NBS Misc. Publ. 270 (July 22, 1965), \$1.25.

Guide to instrumentation literature, J. F. Smith and W. G. Brombacher, NBS Misc. Publ. 271 (July 7, 1965), \$1.25. Supersedes Circ. 567.

Interrelations between cement and concrete properties, Part 1 . Materials, techniques, water requirements and trace elements, R. L. Blaine, H. T. Arni, and B. E. Foster, et al., NBS Bldg. Sci. Series 2, pt. 1 (Aug. 20, 1965), 35 cents.

Quarterly radio noise data March, April, May, 1964, W. Q. Crichlow, R. T. Disney, and M. A. Jenkins, NBS Tech. Note 18-22 (Aug. 2, 1965), 50 cents.

The normal phase variations of the $18 \mathrm{kc} / \mathrm{s}$ signals from NBA observed at College, Alaska, J. H. Crary and A. C. Murphy, NBS Tech. Note 206-4 (Sept. 30, 1965), 25 cents.

The normal phase variations of the $16 \mathrm{kc} / \mathrm{s}$ signals from GBR ob served at College, Alaska, J. H. Crary and A. C. Murphy, NBS Tech. Note 206-5 (Sept. 30, 1965), 30 cents.

HAYSTAQ. A mechanized system for searching chemical informa tion, E. C. Marden, NBS Tech. Note 264 (Sept. 27, 1965), 50 cents.

Accuracy in measurements and calibrations, 1965, W. A. Wildhack, R. C. Powell, and H. L. Mason, NBS Tech. Note 262 (June 15 , 1965) $\$ 1.00$

Tabulation of published data on Soviet electron devices through June 1965, C. P. Marsden, NBS Tech. Note 265 (Oct. 1, 1965), 50 cents. Supersedes NBS Tech. Note 186.

Selected values of chemical thermodynamic properties. Part 1. Tables for the first twenty-three elements in the standard order of arrangement, D. D. Wagman, W. H. Evans, I. Halow, V. B. B. Parker, S. M. Bailey, and R. H. Schumm, NBS Tech. Note 270-1 (Oct. 1, 1965), 65 cents. Supersedes NBS Circ. 500.

Electrochemical analysis: Studies of acids, bases, and salts by EMF conductance, optical and kinetic methods July 1964 to June 1965 , ed. R. G. Bates, NBS Tech. Note 271 (Sept. 6, 1965), 60 cents.

Spectrochemical analysis: Optical spectrometry, x-ray fluorescence spectrometry, and electron probe microanalysis techniques July 1964 to June 1965, ed. B. F. Scribner, NBS Tech. Note 272 (Oct. 21, 1965), 50 cents.

Analysis and purification section: Summary of activities July 1964 to June 1965, ed. J. K. Taylor, NBS Tech. Note 273 (Oct. 1, 1965), 50 cents.

Solubility of solids in dense gases, J. M. Prausnitz, NBS Tech. Note 316 (July 1965), 35 cents.

A numerical representation of CCIR report 322 high frequency (3-30 Mc/s) atmospheric radio noise data, K. L. Lucas and J. D. Harper, Jr., NBS Tech. Note 318 (Aug. 5, 1965), 55 cents.

Boiling heat transfer for oxygen, nitrogen, hydrogen, and helium, E. G. Brentari, P. J. Giarrantano, and R. V. Smith, NBS Tech. Note 317 (Sept. 20, 1965), 60 cents.

Numerical values of the path integrals for low and very low frequencies, L. A. Berry and M. E. Chrisman, NBS Tech. Note 319 (Sept. 2, 1965), 55 cents.

Spark planning damage in copper, J. J. Gniewek, A. F. Clark, and J. C. Moulder, NBS Tech. Note 321, (Sept. 6, 1965), 15 cents.

Surface tensions of normal and parahydrogen, R. J. Corruccini, NBS Tech. Note 322, (Aug. 11, 1965), 20 cents.

Refractive index and dispersion of liquid hydrogen, R. J. Corruccini, NBS Tech. Note 323, (Sept. 24, 1965), 25 cents.

Interference predictions for the instrument landing system, G. D. Gierhart and M. E. Johnson, NBS Tech. Note 324, (Sept. 1965), 30 cents. 
Characteristics of insoluble protein of tooth and bone-I, Fluorescence of some acidic hydrolytic fragments, S. A. Mancewicz and K. C. Hoerman, Arch. Oral. Biol. 9, 535-544 (Oct. 1964).

Electrical properties, A. J. Curtis, Book, Crystalline Olefin Polymers, ed. R. A. V. Raff, 2, 105-130 (Interscience Publ. Inc., New York, N.Y., 1964).

Energy loss straggling of protons and mesons: tabulation of the Vavilov distribution, S. M. Seltzer and M. J. Berger, Natl. Acad. Sci. Natl. Res. Council Publ. 1133. Studies in the Penetration of Charged Particles in Matter, ch. 9, pp. 187-203 (1964).

Emission spectrum of CF, T. L. Porter, D. E. Mann, and N. Acquista, J. Mol. Spectry. 16, 228 (June 1965).

High-pressure x-ray diffraction studies, G. J. Piermarini, Proc. 13th Chemical Engineering Conf., Montreal, Canada, Oct. 19-23, 1963, pp. 182-185 (Chemical Institute of Canada, 1963).

High temperature measurements and standards: $1000-3000{ }^{\circ} \mathrm{C}$, R. F. Walker, (Proc. Intern. Symp. High Temperature Technology, International Union of Pure and Applied Chem., Asilomar, Pacific Grove, Calif., Sept. 1963), Book, High Temperature Technology. Suppl. to Pure and Applied Chem., p. 7 (Butterworths Inc., Washington, D.C., 1964).

$\mathrm{H}_{2}$-transfer reactions in the gas-phase radiolysis of hydrocarbons, P. Ausloos and S. G. Lias, J. Chem. Phys. 43, No. 1, 127-135 (July 1, 1965).

Osmotic and activity coefficients of tris (hydroxymethyl) aminomethane and its hydrochloride in aqueous solution at $25^{\circ} \mathrm{C}, \mathrm{R}$. A. Robinson and V. E. Bower, J. Chem. Engr. Data 10, No. 3, 246-247 (1965).

Oxidation of asphalt in the presence of ozone, J. R. Wright and P. G. Campbell, Am. Chem. Soc. Div. Petroleum Chem. Preprint 9, No. 2, B99 (Apr. 1964).

Oxygen atoms reaction with condensed olefins, R. Klein and M. D. Scheer, Science 144, 1214 (1964).

Present status of project HAYSTAQ, E. C. Marden and H. R. Koller, (Proc. 3rd Annual Meeting Comm. Intern. Cooperation and Information Retrieval Among Examining Patent Offices, Vienna, Austria, Sept. 17-26, 1963), Book, Information Retrieval Among Examining Patent Offices, ed. H. Pfeffer, p. 163 (Spartan Books Inc., Baltimore, Md., 1964).

Radiochemical determination of uranium of low activity, L. A. Currie, G. M. France, III, and P. A. Mullen, Health Phys. 10, 751-755 (1964).

Radiolytic stress relaxation of an ethylene-propylene copolymer, H. Yu and L. A. Wall, J. Phys. Chem. 69, 2072-2078 (June 1965).

Reaction of deuterated polystyrenes with hydrogen and deuterium atoms, L. A. Wall and R. B. Ingalls, J. Chem. Phys. 41, No. 4, 1112-1120 (Aug. 1964).

Sampling of leather for physical and chemical examination, J. Mandel and J. R. Kanagy, Book, The Chemistry and Technology of Leather 4, ch. 59, 223-242 (Reinhold Publ. Corp., New York, N.Y., 1965).

Some electrochemical aspects of germanium dissolution. Simultaneous chemical and electrochemical oxidation, W. E. Reid, Jr., J. Phys. Chem. 69, No. 7, 2269-2277 (July 1965).

Stress relaxation of $\gamma$-irradiated fluorocarbon elastomers, T. Yoshida, R. E. Florin, and L. A. Wall, J. Polymer Sci. A3, No. 5, 1685-1712 (May 1965).

The determination of beta-emitting radionuclides in aqueous formamide solutions, A. Schwebel, Proc. Bioassay and Analytical Chemistry Meeting, Argonne Natl. Lab., Argonne, Ill., Oct. 1962, ANL Publ. 6637, p. 29 (1962).

The half-life of carbon-14; comments on the mass-spectrometric method, E. E. Hughes and W. B. Mann, (Proc. Radio-Carbon Dating Conf., Cambridge, 1962, Intern. J. Appl. Radiation Isotopes 15, 97-100 (1964).

The heats of solution and oxidation of sulfur dioxide, W. H. Johnson and S. Sunner, Acta. Chem. Scand. 17, 1917-1924 (1963).

The kinetics of positive ion desorption from polycrystalline tungsten and rhenium, M. D. Scheer and J. Fine, (Proc. Intern. Symp. Condensation and Evaporation of Solids, Dayton, Ohio, Sept. 12-14, 1962), Book, Condensation and Evaporation of Solids, ed. E. Rutner, P. Goldfinger, and J. P. Huth, Paper 20, p. 327 (Gordon and Breach, New York, N.Y., 1963).

The molecular symmetry of iodopentaborane, L. H. Hall, J. Am. Chem. Soc. Commun. to Editor 86, No. 21, 4729 (Nov. 5, 1964).

The role of carbon dioxide in the bone char process, F. G. Carpenter,
V. R. Deitz, and D. Larry, Proc. 1963 Sugar Industry Technologist 22, No. 2, 27-45 (Chemistry Inst., New York, N.Y., May 1963).

The structure of carbon suboxide, W. J. Lafferty and A. G. Maki, Chemistry 37, No. 7, 19 (July 1964).

Theoretical electromotive forces for cells containing a single solid or molten fluoride, bromide, or iodide, W. J. Hamer, M. S. Malmberg, and B. Rubin, J. Electro. Chem. Soc. 112, No. 7, 750-755 (July 1965).

Thermodynamics of solid carbon dioxide solubility in liquid solvents at low temperatures, A. L. Myers and J. M. Prausnitz, Ind. Engr. Chem. 4, No. 2, 209-212 (May 1965).

Thermogravimetric study of some new transition metal-Schiff base coordination polymers, E. Horowitz, M. Tryon, and R. G. Christensen, J. Appl. Polymer Sci. 9, 2321-2336 (1965).

Weathering: theoretical and practical aspects of asphalt, J. R. Wright, Book, Bituminous Materials: Asphalts, Tars, and Pitches, ed. A. J. Hoiberg, Vol. 2. Asphalts, pt. 1, ch. 8, 249-306 (J. Wiley \& Sons, Inc., New York, N.Y., 1965).

$\mathrm{X}$-ray study of isothermal thickening of lamellae in bulk polyethylene at the crystallization temperature, J. D. Hoffman and J. J. Weeks, J. Chem. Phys. Note 42, No. 2, 4301-4302 (June 15, 1965).

A liquid-medium step-function pressure calibrator, R. O. Smith, J. Basic Eng. Series D, 86, No. 4, 723-727 (Dec. 1964).

Are our neighbors from abroad aware of standards and what standards can do for them? A. T. McPherson, Proc. 13th Annual Meeting Standard Engineering Society, New York, N.Y., Sept. 3, 1964, pp. 11A1-11A2 (1965).

Automatic optical design, O. N. Stavroudis, Book, Advances in Computers, ed. F. L. Alt and M. Rubinoff, IV, 227-255 (Academic Press, Inc., New York, N.Y., 1964).

Clinical evaluation of complete dentures made of 11 different types of denture base materials, J. B. Woelfel, G. C. Paffenbarger, and W. T. Sweeney, J. Am. Dental Assoc. 70, No. 7, 1170-1188 (May 1965).

Comments on the paper "A liquid-medium step-function pressure calibrator," by R. O. Smith, J. L. Cross, Trans. Am. Soc. Mech. Eng. Series D, 86, 729 (1964).

Comments on three papers on fire tests, J. V. Ryan, ASTM Fire Test Symp., Oct. 1, 1962, Am. Soc. Testing Material Spec. Tech. Publ. No. 344, 52 (Oct. 1963).

Digitizing pictorial information with a precision optical scanner, R. T. Moore, M. C. Stark, and L. Cahn, Photogrammetric Eng. XXX, No. 6, 923 (Nov. 1964).

Dimensional changes of dental amalgam associated with strain release in the silver alloy particles, G. Dickson and R. M. Waterstrat, J. Dental Res. 44, No. 3, 620 (May-June 1965).

Discussion of paper 63-39 I, "The measurement of short duration impulse voltages," by F. C. Creed and M. M. C. Collins. H. N. Cones, IEEE Trans. Commun. Electron. EC-69, 629 (Nov. 1963).

Effect of surface texture on diffuse spectral reflectance, Part B. Surface texture measurement of metal surfaces, D. B. Spangenberg, A. G. Strang, and J. L. Chamberlin, (Symp. Measurement of Thermal Radiation of Solids, San Francisco, Calif., Mar. 4-6, 1964), NASA Spec. Publ. SP-55, Session II, Paper 17, pp. 169177 (1965).

Fracture topography of brittle polymers, S. B. Newman, Polymer Engr. Sci. 5, No. 3, 159-165 (July 1955).

Gold in dentistry, W. T. Sweeney and C. L. Burns, American Society for Metals Monograph on Gold. Recovery, Properties and Applications, ch. 13, pp. 227-235 (D. Van Nostrand Co. Inc., Princeton, N. J., 1964).

Guide to dental materials, 1964-1965, G. C. Paffenbarger, J. W. Stanford, and W. T. Sweeney, Am. Dental Assoc. 2d ed., 159 pages (Chicago, Ill., 1964).

Intense resonance line sources for photochemical work in the vacuum ultraviolet region, H. Okabe, J. Opt. Soc. Am. 54, 478 (Apr. 1964).

Interferometer test of the 26-inch refractor at Leander McCormick Observatory, J. B. Saunders, Astron, J. 69, No. 6, 449 (Aug. 1964).

Letter to the Editor of Industrial Research in answer to letters regarding "Synthesis of Food," A. T. McPherson, Ind. Res. Feedback Col. 4, No. 5, 15-16 (May 1962).

Liquid hydrogen bubble chambers, H. P. Hernandez and B. W. Birmingham, Book, Technology and Uses of Liquid Hydrogen, ch. 8, pp. 228-284 (Pergamon Press, Inc., New York, N.Y., 1964). 
Measurement of very-high pressures, E. C. Lloyd and A. A. Giardini, Acta Imeko 2, 305 (1964).

Programmed maneuver-spectrum fatigue test of aircraft beam specimens, L. Mordfin and N. Halsey, Am. Soc. Testing Mater. Spec. Tech. Publ. 338, 251-272 (1963).

Some physical characteristics of agar impression materials, M. Ohashi, G. C. Paffenbarger, and J. W. Stanford, J. Nihon Univ. Sch. Dent. 7, No. 1, 1-11 (Mar. 1965).

Spectral distribution of typical daylight as a function of correlated color temperature, (condensed version), D. B. Judd, Illum. Eng. 60, No. 4, 272-278 (Apr. 1965).

Summary of symposium on methods of metallographic specimen preparation, L. L. Wyman, Am. Soc. Testing Mater. Spec. Tech. Publ. 285, 130 (May 1961).

The cement and concrete reference laboratory inspection service for concrete testing laboratories, J. R. Dise, Concrete Construc. 9, No. 8, 234 (Aug. 1964).

The Institute for Applied Technology, D. A. Schon, Engineer V, No. 2, 3 (Summer 1964).

The liquefaction of hydrogen. 3A. Basic principles, P. C. Vander Arend and D. B. Chelton, Book, Technology and Uses of Liquid Hydrogen, ch. 3, pp. 38-55 (Pergamon Press, Inc., New York, N.Y., 1964).

The liquefaction of hydrogen. 3B-2. Large scale hydrogen liquefaction facilities, P. C. Vander Arend and D. B. Chelton, Book, Technology and Uses of Liquid Hydrogen, ch. 3, pp. 79-105 (Pergamon Press Inc., New York, N.Y., 1964).

The work of NOMAD-1, W. Hakkarinen, Proc. Buoy Technology Symp., Washington, D. C., Mar. 24-25, 1964, p. 443 (Marine Technology Society, 1964).

What is needed next in the simulation of the arterial street? M. C. Stark, Proc. Highway Conf. Progress Related to the Future of Surveillance, Simulation and Control, Washington, D.C., Sept. 14-15, 1964, p. 144 (Bureau of Public Roads, Washington, D.C. 1964).

What's new in control standards, H. L. Mason, Control Eng. 11, No. 6, 85-87 (June 1964).

Address encoding - A study of the double-binary keyboard as a link in the machine-sorting of mail, J. R. Cornog, J. F. Hockman, and J. C. Craig, (Proc. Winter Annual Meeting, American Society of Mechanical Engineers, Philadelphia, Pa., Nov. 17-22, 1963), ASME Publ. 63-WA-338 (1964).

How people influence experimental results, J. R. Cornog, Proc. Social Statistics Section Convention, American Statistical Association, Cleveland, Ohio, pp. 119-122, (1964).

Mortality patterns in eight strains of flour beetles, D. B. Mertz, T. Park, and W. J. Youden, Biometrics 21, No. 1, 99-144 (Mar. 1965).

Plasma oscillations with collective correlations, C. M. Tchen, Proc. 6th Intern. Conf. Ionization Phenomena in Gases, Paris, France, July 8-13, 1963, I, 195-199 (P. Hubert Assoc, EURATOM-CEA Centra-d-Etudes Nucleaires de Fontenay-aux-Roses, Paris, France, 1963).

The current status of graphic storage techniques: their potential application to library mechanization, S. N. Alexander, (Proc. Conf. Library Automation, Airlie Foundation, Warrington, Va., May 26-30, 1963), Book, Libraries and Automation, ed. B. E. Markuson, pp. 111-140 (Library of Congress, Washington, D.C., 1964).

Accurate length measurement of meter bar with helium-neon laser, K. D. Mielenz, H. D. Cook, K. E. Gillilland, and R. B. Stephens, Science Letter 146, No. 3652, 1672-1673 (Dec. 1964).

Comments on "Application of experimental design to the study of a test method," R. T. Thaxter and P. D. Hubbe. J. Mandel, Tappi i5. No. 12, 193A-194A (Dec. 1963).

Direct quantitative analysis of microstructures by a digital computer, G. A. Moore, Am. Soc. Metals Tech. Report No. 16.3 (1964); (condensed version), Book, Computer Applications in Metallurgical Engineering, p. 111 (Am. Soc. Metals, Metals Park, Ohio, 1964).

Educating metrologists, T. R. Young, Ordnance XLIX, No. 268, 432-435 (Jan.-Feb. 1965).

Measurement-R \& D looks to future, W. A. Wildhack, Control Eng. 12, No. 1, 75-76 (Jan. 1965).

Metrology as related to microminiaturization processes, T. R. Young, Proc. Microminiaturization Congress, New York, N.Y., Mar.
10-13, 1962 (American Watchmakers Inst., New York, N.Y., Mar. 1963).

The definition of alginate impression materials by a specification, M. Ohashi, G. C. Paffenbarger, and J. W. Stanford, J. Nihon Univ. School Dent. 7, No. 1, 12-28 (Mar. 1965).

The stistical construction of a single standard from several available standards, E. L. Crow, IEEE Trans. Instr. Meas. IM-13, No. 4, 180-185 (Dec. 1964).

Atomic scattering factors for the lithium and beryllium isoelectronic sequences from accurate analytic Hartree-Fock wave functions, C. M. Womack, Jr., and J. N. Silverman, Z. Kryst. 118, No. 5-6, 422-433 (Sept. 1963).

Calculation of electronic energies in $\mathrm{HCO}_{2}^{+}, \mathrm{HCO}_{2}$ and $\mathrm{HCO}_{+}^{+}$, W. H. Kirchhoff, J. Farren, and J. W. Linnett, J. Chem. Phys. 42, No. 4, 1410-1422 (Feb. 15, 1965).

Calculations of the hyperfine splitting for the lithium atom, J. B. Martin and A. W. Weiss, J. Chem. Phys. Letter 39, No. 6, 16181620 (Sept. 15, 1963).

Calculation of the radiative tail in the inelastic scattering of electrons, L. C. Maximon and D. B. Isabelle, Proc. Intern. Conf. Nuclear Physcis, Paris, France, July 2-8, 1964, 2, 1031-1033 (1964).

Coupling of particle-hole states and two particle-two hole states: splitting, W. Greiner and M. Danos, Compt. Rend. Congres Intern. Physique Nucleaire, ed. P. Gugenberger, II, 617 (Editions du Centre National de la Recherche Scientific, Paris, France, 1964).

Connection between shielding and stability in a collisionless plasma, F. Engelmann, M. Feix, and E. Minardi, Il Nuovo Cimento, Serie X, 30, 830-836 (Nov. 1963).

Dynamic theory of the nuclear collective model, M. Danos and W. Greiner, Phys. Rev. 134, B285 (1964).

Experimental verification of the WLF superposition technique, A. J. Bur, Proc. Electrical Insulation Conf., Cleveland, Ohio, Oct. 1964, Natl. Acad Sci.-Natl. Res. Council Publ. 1238, pp. 70-71 (1965).

Experimental fires in enclosures, D. Gross and A. F. Robertson, Proc. 10th Symp. (Intern.) Combustion, Cambridge, England, Aug. 1964, pp. 931-942 (1965).

General report on fundamental spectroscopic data, C. E. Moore (Proc. Intern. Astronomical Union Commission 14 Meeting, Hamburg, Germany, Aug. 1964), Trans. Intern. Astron. Union XIIA, 156 (July 1965).

Gas mixtures and pressures for optimum output power of RF excited helium-neon gas lasers at $632.8 \mathrm{~nm}, \mathrm{~K}$. D. Mielenz and K. F. Nefflen, Appl. Opt. Letter 4, 565 (May 1965).

Impurity controlled properties of ionic solids, A. D. Franklin (Proc. Symp. Physics and Chemistry of Ceramics, Pennsylvania State Univ., University Park, Pa., May 28-30, 1963), Book, Physics and Chemistry of Ceramics, pp. 77-109 (Gordon and Breach, New York, N.Y., 1963).

Hypervelocity cratering data and a crater depth model for the regime of partical fluidity, O. G. Engel, Proc. 6th Hypervelocity Impact Symp., Cleveland, Ohio, Apr. 30-May 1, 1963, II, pt. II, 337-366 (U.S. Army Ballistics Res. Lab., Aberdeen Proving Ground, Md., Aug. 1963).

Introduction, Book, Technology and Uses of Liquid Hydrogen, R. B. Scott, pp. 1-8 (Pergamon Press Ltd., London, England, 1964).

Laboratory measurement of the rate of the reaction $\mathrm{O}^{+}+\mathrm{O}_{2} \rightarrow \mathrm{O}_{2}^{+}+\mathrm{O}$ at thermal energy, F. C. Fehsenfeld, P. D. Goldan, A. L. Schmeltekopf, and E. E. Ferguson, Planet. Space Sci. 13, 579-582 (Mar. 1965).

Minutes of the Meeting at Tokyo, Japan, of the Triple Commission for Spectroscopy, September 9, 1962, C. E. Moore, J. Opt. Soc. Am. 53, No. 7, 883-893 (July 1963).

Nuclear magnetic resonance in bulk nickel samples, L. H. Bennett, Phil. Mag. 12, No. 115, 213-215 (July 1965).

On the possibility of the measurement of the nuclear $2^{4}$-pole deformation, M. Danos, W. Greiner, and C. G. Kohr, Physics Letters 12 , No. 4, 344 (1964).

Optical constants in the vacuum ultraviolet and electron energy losses, L. L. Marton, J. Quant. Spectr. Radiative Transfer 2, 671-678 (May 1964)

Preliminary studies on the characterization of solution-grown ADP crystals, R. D. Deslattes, J. L. Torgensen, B. Paretzkin, and A. T. Horton, Advan. X-Ray Anal. 8, 315-324 (1965).

Relation of the stacking fault energy to segregation at stacking faults and to the occurrence of phase boundaries in F. C. C. binary alloys, R. DeWit and R. E. Howard, Acta Met. 13, 655-661 (June 1965). 
The determination of work function from the ratio of positive to negative surface ionization of an alkali halide, J. Fine, T. E. Madey and M. D. Scheer, Surface Sci. 3, 227 (1965).

The gamma-ray distribution from oriented cerium-141 and its application to thermal contact at low temperature, J. F. Schooley and D. D. Hoppes (Proc. 8th Intern. Conf. Low Temperature Physics, Queen Mary College, London, England, Sept. 1963), Book, Low Temperature Physics, ed. R. O. Davies, pp. 435-436 (Butterworth, Inc., Washington, D.C., 1963).

The spin lattice relaxation time of some paramagnetic dispersions, P. H. Fang (Proc. XI Colloque Ampere, Eindhoven, The Netherlands, June 1962), Book, Magnetic and Electric Resonance and Relaxation, pp. 123-128 (1963).

Theoretical aspects of polymer crystallization with chain folds: bulk polymers, J. D. Hoffman, SPE Trans. 4, No. 4, 315-362 (Oct. 1964).

Utility of the Tait equation relating volume and pressure in the study of transitions in polymers, L. A. Wood, J. Polymer Sci. B2, No. 7, 703-707 (July 1964).

Unorthodoxy in science, L. A. Wood, J. Wash. Acad. Sci. 51, 30 (Mar. 1961)

Vapor-phase growth kinetics of potassium and mercury crystals, R. L. Parker and S. C. Hardy, (Proc. Intern. Symp. Condensation and Evaporation of Solids, Dayton, Ohio, Sept. 12-14, 1962), Book, Condensation and The Evaporation of Solids. Section II. Condensation of Solids, p. 649 (Gordon and Breach, New York, N.Y., 1964).

Volume relaxation of $\mathrm{As}_{2} \mathrm{O}_{3}$ in the glass transformation, A. B. Bestul, Proc. Intern. Conf. on Physics of Non-Crystalline Solids, Delft, The Netherlands, July 1964, pp. 426-435 (North-Hollard Publ., Amsterdam, The Netherlands, 1965).

Volume relaxation in amorphous polymers, R. S. Marvin and J. E. McKinney, Book, Physical Acoustics, ed. W. P. Mason, IIB, ch. 9, 165-229 (Academic Press Inc., New York, N.Y., 1965).

Dependence of the critical frequency of the ionospheric E-layer on solar zenith angle and the annual variation in $E$-layer ionization, T. Shimazaki, Nature 205, No. 4974, 889-891 (Feb. 27, 1965).

Justification for neglecting the compressibility of the ionosphere in VLF radio propagation, J. R. Wait, IEEE Trans. Ant. Prop. AP-13, 480-481 (May 1965).

National Bureau of Standards Central Radio Propagation Laboratory, Boulder, C. G. Little, Astron. J. 69, No. 9, 698-700 (Nov. 1964) Natural electromagnetic phenomena below $30 \mathrm{kc} / \mathrm{s}$, R. M. Gallet, NATO Advanced Study Institute, Bad Hamberg, pp. 167-204 (Plenum Press Inc., New York, N.Y., 1964).

Propagation and technical factors in radio spectrum utilization, J. W. Herbstreit, Radio Spectrum Utilization, ed. J. W. Herbstreit, A report of the Joint Tech. Adv. Comm. of Inst. Elec. Electron. Eng. Inc., and the Electron Ind. Assoc., ch. III, pp. 30-208 (1964). 30-208 (1964).

Representation of diurnal and geographic variations of ionospheric data by numerical methods. II. Control of stability, W. B. Jones and R. M. Gallet, Telecommun. J. pp. 1-12 (Place des Nations, Geneva, Switzerland, Jan. 1965).

The ionosphere; new guide, how to listen to the world, A. H. Shapley, World Publ. 11, 15-18 (Hellerup, Denmark, Mar. 1965).

Adhesive bonding of various materials to hard tooth tissues. I. Method of determining bond strength, R. L. Bowen, J. Dental Res. 44, No. 4, 690-695 (July-Aug. 1965).

Crystal growth as chemical research, H. S. Peiser and J. L. Torgesen, Chemistry 38, 15-20 (1965).

Determining moisture in solid materials by reaction with calcium carbide, S. Dahl, Mater. Res. Std. 5, No. 9, 446-453 (Sept. 1965).

Dissociation constant of acetic acid in deuterium oxide from 5 to $50^{\circ}$. Reference points for a $p \mathrm{D}$ scale, R. Gary, R. G. Bates, and R. A. Robinson, J. Phys. Chem. 69, No. 8, 2750-2753 (1965).

Infrared spectrum of the free radical NCN, D. E. Milligan, M. E. Jacox, J. J. Comeford, and D. E. Mann, J. Chem. Phys. 43, No. 2, 756-757 (July 15, 1965).

Mass spectrometric study of the reaction of nitrogen atoms with ethylene, J. T. Herron, J. Phys. Chem. 69, No. 8, 2736-2740 (Aug. 1965).

Frequency-dependent transport coefficients in fluid mechanics, R. Zwanig, J. Chem. Phys. 43, No. 2, 714-720 (July 15, 1965).

Thermal decomposition of 2,3-dimethylbutane in a single-pulse shock tube, W. Tsang, J. Chem. Phys. 43, No. 2, 352-359 (July $15,1965)$.
Xenon photosensitized formation of metastable nitrogen, M. D. Scheer and W. M. Jackson, Science 148, 3678 (1965).

Focused-beam electron bombardment evaporator, D. H. Blackburn and W. Haller, Rev. Sci. Instr. 36, No. 7, 901-903 (July 1965).

The effect of environment on the fatigue strengths of four selected alloys, T. R. Shives and J. A. Bennett, NASA Contractor Report CR-267, 2-22 (Aug. 1965).

The temporal dimensional stability of surface-hardened steels, M. R. Meyerson, Metal Treating 15, No. 6, 5 (Dec. 1964-Jan. 1965). Analysis of the spectrum of triply ionized praseodymium ( $P r$ IV), J. Sugar, J. Opt. Soc. Am. 55, No. 9, 1058-1061 (Sept. 1965).

A method for measuring polarization in the vacuum ultraviolet, $\mathrm{K}$. Rabinovitch, L. R. Canfield, and R. P. Madden, Appl. Opt. 4, No. 8, 1005-1010 (Aug. 1965).

A one-dimensional model of polymer adsorption, E. A. DiMarzio and F. L. McCrackin, J. Chem. Phys. 43, No. 2, 539-547 (July 1965).

Drift mobility and diffusion for impurities in ionic crystals, J. R. Manning, Phys. Rev. 139, No. 6A, A2027-A2034 (Sept. 13, 1965).

Energy levels of polarons in a magnetic field, D. M. Larsen, Phys. Rev. 135, No. 2A, A419-A426 (July 20, 1964).

Excitation of optically forbidden states in the ionization continuum by electron impact, J. A. Simpson, G. E. Chamberlain, and S. R. Mielczarek, Phys. Rev. 139, No. 4A, A1039-A104l (Aug. 1965).

Experiments in electron scattering, L. L. Marton, Lab. Invest. 14, No. 6, pt. II, 754 (1965).

Historical background of image formation, L. Marton, Lab. Invest. 14, No. 6, pt. I, 739 (1965).

Investigation of the anomaly in the response of silicon semiconductor radiation detectors at low temperatures, W. R. Dodge, S. R. Domen, A. T. Hirshfeld, and D. D. Hoppes, IEEE Trans. Nuclear Sci. NS-12, 295 (Feb. 1965).

Letter to the Editor "Physical significance of the Fulcher equation," A. B. Bestul, Phys. Chem. Glasses 6, No. 3, 108-110 (June 1965). Matter transport in solids, R. E. How ard and A. B. Lidiard, Rept. Progr. Phys. XXVII, 161-240 (1964).

Optical properties of beryllium in the ultraviolet from electron energy absorption, R. E. LaVilla and H. Mendlowitz, Appl. Opt. 4, No. 8, 955-960 (Aug. 1965).

The annealing of vacancies in dilute alloys, R. E. Howard and A. B. Lidiard, Phil. Mag. 11, No. 114, 1179-1187 (June 1965).

Thermal diffusion of substitutional impurities in metals, R. E. Howard and A. B. Lidiard, Acta Met. 13, No. 4, 433-444 (1965).

Buffer solutions of tris (hydroxymethyl) aminomethane for $p \mathrm{H}$ control in 50 weight percent methanol from $10^{\circ}$ to $40^{\circ} \mathrm{C}$, M. Woodhead, M. Paabo, R. A. Robinson, and R. G. Bates, Anal. Chem. 37, 1291 (1965).

Electrode potentials in fused systems. IX. Liquid potentials in the $\mathrm{AgCl}-\mathrm{AgBr}$ system, K. H. Stern, J. Electrochem. Soc. 112, No. 10, 1049-1050 (Oct. 1965).

Isotopic mixing in nitrogen chemisorbed on W, J. T. Yates, Jr., and T. E. Madey, J. Chem. Phys. 43, No. 3, 1055-1059 (Aug. 1, 1965). Matrix isolation study of the reaction of $\mathrm{Cl}$ atoms with $\mathrm{CO}$. The infrared spectrum of the free radical ClCO, M. E. Jacox and D. E. Milligan, J. Chem. Phys. 43, No. 3, 866-870 (Aug. 1, 1965). Some electrochemical aspects of germanium dissolution. Valence states and electrode potential, W. E. Reid, Jr., Phys. Chem. 69, No. 9, 3168-3171 (Sept. 1965).

Theoretical electromotive forces for cells containing a single solid or molten oxide, W. J. Hamer, J. Electroanal. Chem. 10, 140-150 (1965).

A pulsed refrigeration system for cryogenic magnet application, T. R. Strobridge and D. B. Mann. (Proc. 1964 Cryogenic Engineering Conf., Univ. Pennsylvania, Phila., Pa., Aug. 18-24, 1964), Book, Advances in Cryogenic Engineering 10, Paper N-3, 54-61 (Plenum Press Inc., New York, N.Y., 1965).

An anomalous decrease of the elastic moduli at very low temperatures in some 300 series stainless steels, R. P. Reed and R. P. Mikesell (Proc. 1964 Cryogenic Engineering Conf., Univ. Pennsylvania, Phila., Pa., Aug. 18-24, 1964), Book, Advances in Cryogenic Engineering 10, Paper A-5, 46-49 (Plenum Press, Inc., New York, N.Y., 1965).

Avoiding errors from stray radiation in measuring the spectral emittance of diffusely reflecting specimens, H. E. Clark, Appl. Opt. Letter to Editor 4, No. 10, 1356-1357 (Oct. 1965).

Calibration of Bell-type provers by bottling and strapping, C. T. Collett (Proc. 24th Annual Appalachian Gas Measurement Short Course, 1964), West Virginia Univ. Tech. Bull. 73, 59-65 (1965). 
Calibration of microphones, vibration pickups, and earphones, R. K. Cook, S. Edelman, and W. Koidan, J. Audio Engr. Soc. 13, No. 4, 290-296 (Oct. 1965).

Considerations when using turbine-type flow-meters in cryogenic service, W. J. Alspach and T. M. Flynn (Proc. 1964 Cryogenic Engineering Conf., Univ. Pennsylvania, Phila., Pa., Aug. 18-24, 1964), Book, Advances in Cryogenic Engineering 10, Paper F-3, 246-252 (Plenum Press, Inc., New York, N.Y., 1965).

Four methods of determining temperature sensitivity of strain gages at elevated temperatures, C. H. Melton and R. L. Bloss, ISA J. 12, No. 10, 69-74 (Oct. 1965).

International comparison of dielectric measurements, H. E. Bussey, J. E. Gray, E. C. Bamberger, E. Rushton, G. Russell, B. W. Petley, and D. Morris, IEEE Trans. Instr. Meas. IM-13, No. 4, 305-311 (Dec. 1964).

Nucleate and film pool boiling design correlations for $\mathrm{O}_{2}, \mathrm{~N}_{2}$, and He, E. G. Brentari and R. V. Smith (Proc. 1964 Cryogenic Engineering Conf., Univ. Pennsylvania, Phila., Pa., Aug. 18-24, 1964), Book, Advances in Cryogenic Engineering 10, Paper T-1, 325341 (Plenum Press, Inc., New York, N.Y.., 1965).

Production of alumina windows, H. Johnson and R. D. Deslattes, Rev. Sci. Instr. 36, No. 9, 1381-1382 (Sept. 1965).

Solid-vapor equilibrium in the system hydrogen-methane, M. J. Hiza and R. N. Herring (Proc. 1964 Cryogenic Engineering Conf., Univ. Pennsylvania, Phila., Pa., Aug. 18-24, 1964), Book, Advances in Cryogenic Engineering 10, Paper Q-5, 182-191 (Plenum Press, Inc., New York, N.Y., 1965).

Transfer line surge, W. G. Steward (Proc. 1964 Cryogenic Engineering Conf., Univ. Pennsylvania, Phila., Pa., Aug. 18-24, 1964), Book, Advances in Cryogenic Engineering 10, Paper I-3, 313-322 (Plenum Press, Inc., New York, N.Y., 1965).

The international system of units, A. G. McNish, Mater. Res. Stds. 5, No. 10, 528-532 (Oct. 1965).

DC dielectric conductance (reciprocal resistance) and conductivity (reciprocal resistivity) measurements, A. H. Scott, Book, Testing of Polymers 1, ch. 6, 213-236 (Interscience Publ., Inc., New York, N.Y., 1965).
Dispersion characteristics of the $1.15 \mu \mathrm{He}-\mathrm{Ne}$ laser line, H. S. Boyne, M. M. Birky, and W. G. Schweitzer, Jr., Appl. Phys. Letters 7, No. 3, 62-65 (Aug. 1, 1965).

Inelastic scattering of electrons by helium, G. E. Chamberlain and H. G. M. Heideman, Phys. Rev. Letters 15, No. 8, 337-338 (Aug. 1965).

Infrared measurements on allene and allene- $\mathrm{d}_{4}$, A. G. Maki and R. A. Toth, J. Mol. Spectr. 1 7, No. 1, 136-155 (July 1, 1965).

Nuclear resonance spin-echo study of $\mathrm{Eu}^{153}$ in EuO, G. A. Uriano and R. L. Streever, Physics Letters 17, No. 3, 205-206 (July 15, 1965).

Nuclear resonance study of gallium-substituted yttrium iron garnet, R. L. Streever and G. A. Uriano, Phys. Rev. 139, No. 1A, A305A313 (July 5, 1965).

Nuclear resonance study of hyperfine fields in nickel-rich nickelcobalt alloys, R. L. Streever and G. A. Uriano, Phys. Rev. 139, No. 1A, A135-Al41 (July 5, 1965).

Results of an absolute determination of the acceleration of gravity, J. E. Faller, J. Geophys. Res. 70, No. 16, 4035-4038 (Aug. 15, 1965).

Book, Some aspects of non-equilibrium thermodynamics in the presence of a radiation field, R. N. Thomas (University of Colorado Press, Boulder, Colo., 1965).

Thermal conductivity and thermoelectric power of rutile $\left(\mathrm{TiO}_{2}\right)$, W. R. Thurber and A. J. H. Mante, Phys. Rev. 139, No. 5A, Al655Al665 (Aug. 30, 1965).

Nature of the electromagnetic field reflected from a coastline, J. R. Wait, Electronics Letters 1, 65 (May 1965).

*Publications for which a price is indicated are available by purchase from the Superintendent of Documents, U.S. Government Printing Office, Washington, D.C., 20402 (foreign postage, onefourth additional). Reprints from outside journals and the NBS Journal of Research may often be obtained directly from the authors. 